\title{
In vivo dosimetry using MOSFET detectors in radiotherapy
}

Citation for published version (APA):

Bloemen - van Gurp, E. J. (2009). In vivo dosimetry using MOSFET detectors in radiotherapy. [Doctoral Thesis, Maastricht University]. Datawyse / Universitaire Pers Maastricht. https://doi.org/10.26481/dis.20091030eb

Document status and date:

Published: 01/01/2009

DOI:

10.26481/dis.20091030eb

Document Version:

Publisher's PDF, also known as Version of record

\section{Please check the document version of this publication:}

- A submitted manuscript is the version of the article upon submission and before peer-review. There can be important differences between the submitted version and the official published version of record.

People interested in the research are advised to contact the author for the final version of the publication, or visit the DOI to the publisher's website.

- The final author version and the galley proof are versions of the publication after peer review.

- The final published version features the final layout of the paper including the volume, issue and page numbers.

Link to publication

\footnotetext{
General rights rights.

- You may freely distribute the URL identifying the publication in the public portal. please follow below link for the End User Agreement:

www.umlib.nl/taverne-license

Take down policy

If you believe that this document breaches copyright please contact us at:

repository@maastrichtuniversity.nl

providing details and we will investigate your claim.
}

Copyright and moral rights for the publications made accessible in the public portal are retained by the authors and/or other copyright owners and it is a condition of accessing publications that users recognise and abide by the legal requirements associated with these

- Users may download and print one copy of any publication from the public portal for the purpose of private study or research.

- You may not further distribute the material or use it for any profit-making activity or commercial gain

If the publication is distributed under the terms of Article $25 \mathrm{fa}$ of the Dutch Copyright Act, indicated by the "Taverne" license above, 


\section{In vivo dosimetry using MOSFET detectors in radiotherapy}

Esther Bloemen- van Gurp 


\section{Production}

Cover: $\quad$ E. Bloemen- van Gurp

Printing: $\quad$ Datawyse | Universitaire Pers Maastricht ISBN: $\quad 9789052788579$

\section{Copyrights}

(c) Copyright: $\quad$ E. Bloemen- van Gurp, Maastricht 2009

Chapter 2: $\quad$ Radiother Oncol, Elsevier Ireland Ltd, 2003

Chapter 3 Radiother Oncol, Elsevier Ireland Ltd, 2006

Chapter 4: $\quad$ Int J Radiat Oncol Biol Phys, Elsevier Inc, 2007

Chapter 5: $\quad$ Int J Radiat Oncol Biol Phys, Elsevier Inc, 2009

Chapter 6: $\quad$ Int J Radiat Oncol Biol Phys, Elsevier Inc, 2009

No part of this publication may be reproduced in any form without prior written permission of the holder of the copyrights. 


\title{
In vivo dosimetry using MOSFET detectors in radiotherapy
}

\author{
Proefschrift \\ ter verkrijging van de graad van doctor \\ aan de Universiteit Maastricht, \\ op gezag van de Rector Magnificus \\ Prof. mr. G.P.M.F. Mols, \\ volgens het besluit van het College van Decanen, \\ De openbare verdediging zal plaatsvinden op \\ vrijdag 30 oktober 2009 om 14.00 uur \\ door \\ Esther Jacobien Bloemen- van Gurp
}

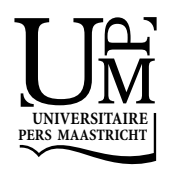




\section{Promotor}

Prof. dr. Ph. Lambin

\section{Copromotores}

Dr. B.J. Mijnheer

Dr. ir. A.L.A.J. Dekker

\section{Beoordelingscommissie}

Prof. dr. J.E. Wildberger (voorzitter)

Prof. dr. B.J.M. Heijmen (Erasmus MC)

Dr. Ir. J.L.M. Venselaar (Instituut Verbeeten)

Prof. dr. F.Verhaegen

\section{Paranymfen}

Dr. J.J Jager

Dr. L.P. Murrer

The printing of this thesis was partially supported by:

PI Medical Diagnostic Equipment B.V, The Netherlands, C.Curie Diagnostic Equipment, Belgium and Siemens Nederland NV. 


\section{CONTENTS}

General

List of abbreviations

\section{Introduction}

Chapter 1 Introduction and outline of the thesis

In vivo dosimetry in external beam radiotherapy

Chapter 2 Clinical dosimetry with MOSFET dosimeters to determine the dose along the field junction in a split beam technique

Chapter 3 Clinical implementation of MOSFET detectors for dosimetry in electron beams

Chapter 4 Total body irradiation, towards optimal individual delivery: dose evaluation with MOSFETs, TLDs and a treatment planning system

\section{In vivo dosimetry in brachytherapy}

Chapter 5 In vivo dosimetry using a linear MOSFET array dosimeter to determine the urethra dose in ${ }^{125}$ permanent prostate implants

Chapter 6 Real-time dosimetry with a linear MOSFET array to evaluate the urethra dose during permanent implant brachytherapy using iodine-125

\section{Discussion}

Chapter 7 General Discussion and Future Perspectives 
Summary

Samenvatting

Dankwoord

Curriculum Vitae

Publications

159

Achievements and implementations

163 


\section{LIST OF ABBREVIATIONS}

\section{General}

3D 3-dimensional

125! lodine-125

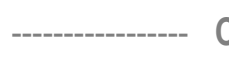

CF Correction factor

$\mathrm{Ci} \quad$ curie

CT Computed tomography

d Diameter

D Accumulated dose

$\dot{D} \quad$ Dose-rate

Dchamber Midline ionization chamber dose values

$D_{\text {entrance }} \quad$ Dose recalculated to $Z_{\max }$ and corrected for non-reference conditions

DGRT Dose guided radiation therapy

$D_{\max } \quad$ Dose at the depth of dose maximum

Dmidline Midline dose value

Dnew Dose-rate after the implantation of a new train of seeds

$D_{\text {plan }} \quad$ Prescribed dose

$\mathrm{DRa} \quad$ Dose-rate before the implantation of the last needle

DRact Actual dose-rate

$\mathrm{DRb} \quad$ Dose-rate after the implantation of the last needle

DRmax Maximum tolerable dose-rate for the urethra

DRmin Minimum dose-rate

DRrec Dose-rate measured in the recovery, directly after the implantation

DRrt Dose-rate real-time

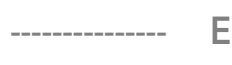

$\begin{array}{ll}\text { E } & \text { Energy } \\ \text { EPID } & \text { Electronic Portal Imaging Device }\end{array}$ 
$\mathrm{F}$

$\begin{array}{ll}\mathrm{F}_{\text {cal }} & \text { Calibration factor } \\ \mathrm{F}_{\text {conversion }} & \text { Conversion factor } \\ \text { FFTC } & \text { Fast-Fourier Transform Convolution }\end{array}$

G

Gy gray

H

HDR High Dose Rate

I ---------------

Ids $\quad$ Current between the source and the drain

IDR Initial Dose Rate

IMRT Intensity-Modulated Radiation Therapy

LDR Low Dose Rate

Linac Linear electron accelerator

M

MBRT Opleiding Medisch Beeldvormende en Radiotherapeutische Technieken

MGS Multi-Grid Superposition

MLC Multileaf collimator

MOSFET Metal-Oxide Semiconductor Field Effect Transistor

MU Monitor unit

OAR Organ at risk

PDD Percentage Depth Dose

PMMA Polymethylmethacrylaat

QA Quality assurance 


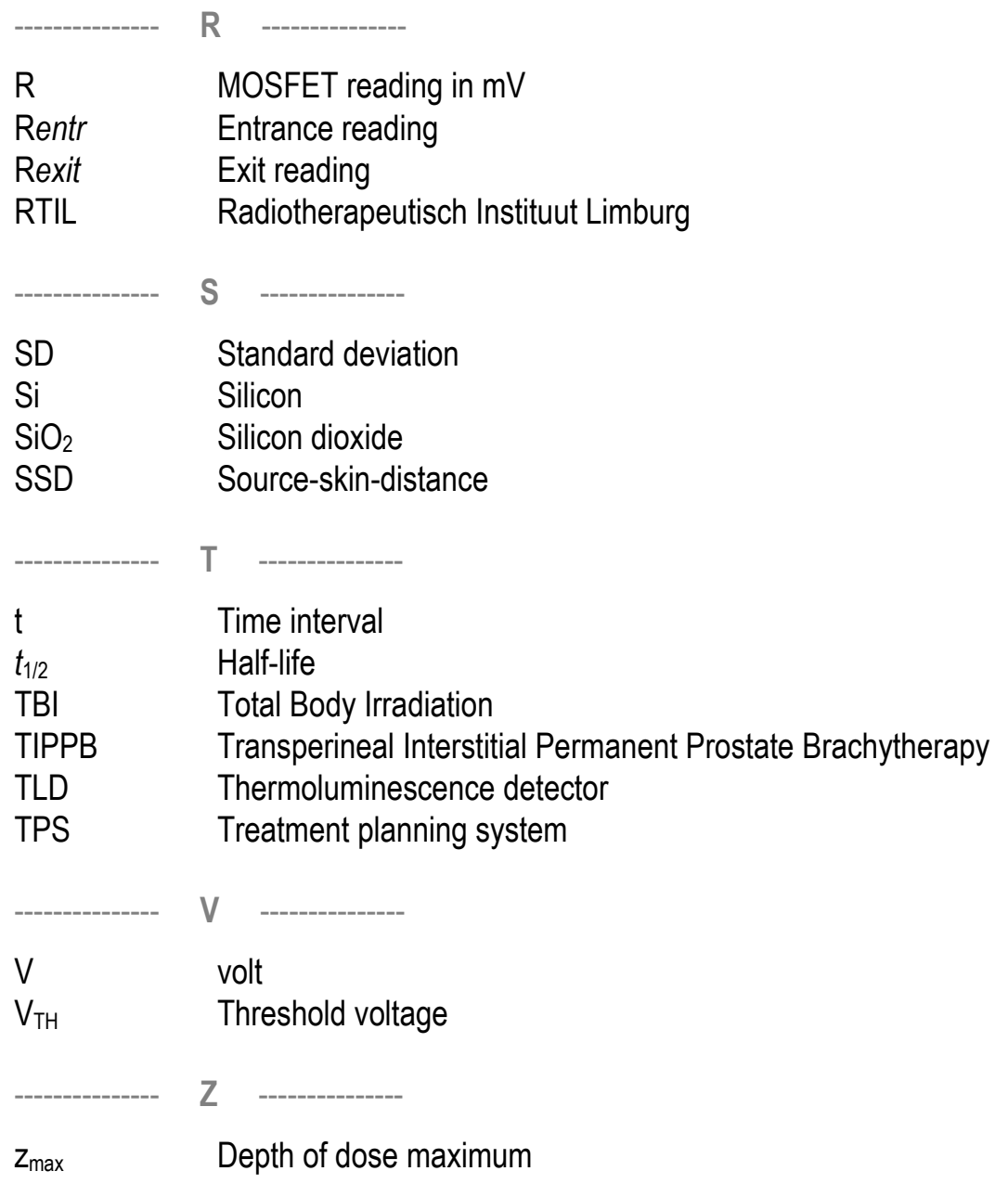



$\equiv$

$\equiv$

\section{Introduction Introduction} . 을 e

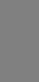



CHAPTER

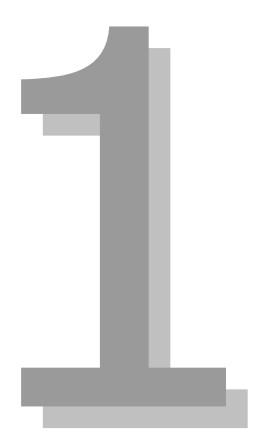

Introduction and outline of the thesis 



\subsection{INTRODUCTION}

The incidence of cancer in Europe is growing over the last decades. With an estimated 3.2 million new cases (53\% occurring in men, $47 \%$ in women) and 1.7 million deaths (56\% in men, $44 \%$ in women) each year, cancer generates an important public health problem in Europe and the ageing of the European population will cause these numbers to continue to increase even if age-specific rates remain constant ${ }^{1}$.

Radiotherapy is an important treatment option for a large range of cancer sites. It uses ionizing radiation to damage the cancer cells, and stop them from growing and dividing. The radiation can be administered by external beam radiotherapy, where an external source of radiation is pointed at a particular part of the body, or by brachytherapy, where a radioactive source is placed inside or next to the volume requiring treatment. Sometimes a combination of these two techniques is applied.

External beam radiotherapy is delivered by an external source of radiation, for which generally high energy photon or electron beams are used, generated by a linear electron accelerator (linac). Photon beams are produced by the rapid deceleration of electrons in a target material. Before exiting the treatment head, the generated photons pass through several components, which influence the production, shaping, localization, and monitoring of the clinical photon beams. These clinical high energy photon beams penetrate within the patient's body and are used to treat deep-seated target volumes. The energy deposition of a megavoltage photon beam in tissue is characterized by a relatively low surface (skin) dose, in the range of $10 \%-25 \%$ of the maximum dose. When the radiation further passes into tissue, dose values increase up to a maximum value and then gradually decrease at increasing distance beneath this point, because of the beam attenuation in tissue and the inverse square law. Typical central axis depth dose curves in water for 6 and $10 \mathrm{MV}$ photon beams, are illustrated in figure 1a.

A large number of techniques using photon beams have been developed for the treatment of tumors. Most techniques use multiple beams, all directed towards the tumor generally applying a fixed distance from the target (source) in the linac to the centre of the tumor. Field sizes range from very small fields used in radiosurgery and during Intensity-Modulated Radiation Therapy (IMRT) techniques, standard rectangular and irregular fields and very large fields used for special indications where the treatment volume is large. For some indications, however, the volume to be irradiated is too large for the field size that can be created by a linac at the standard treatment distance. To increase the maximum field size for these kinds of treatments, normally the source-skin-distance (SSD) is extended. An example of such an extended field technique is Total Body Irradiation (TBI). TBI involves radiation of the whole body and is used for a large variety of clinical situations, such as hematological malignancies and aplastic anemia. The dose distribution during a TBI treatment is generally assumed to be homogeneous and within $10 \%$ of the prescribed dose value ${ }^{2}$. Most treatment 
planning systems (TPS) do not allow the setup of patients at a very large SSD and dose calculations are performed by a point-dose determination at the dose prescription point. As a result the dose distribution in the other parts of the body is not evaluated and rather large uncertainties in the relative dose distribution in the patient's body may occur. To achieve dose uniformity throughout the body, the use of bolus, partial attenuators or compensators is necessary. For the design of these compensators, detailed information of dose delivery to the whole body is required and a detailed calculation of the absorbed dose in the whole body, obtained by a Computed Tomography (CT)- based treatment planning, is essential. A comprehensive quality assurance (QA) programme is therefore necessary to test a TPS for dose calculations of these special treatment techniques.

Situations where the patient's contour and normal tissue constraints require more than one beam configuration also require a special treatment technique. In these treatments, a junction of fields is sometimes unavoidable. A number of techniques have been developed to achieve dose uniformity in the area of the field junction, such as using split beams. This is achieved by placing a half-beam block along a plane through the central axis, thus removing the geometric divergence of the beams at the split line. In theory this technique results in an optimal dose distribution in the junction area. In practice however, several uncertainties affect the accuracy of this technique, originating from factors related to mechanical properties in combination with the QC procedures and the adjustments of the linear accelerator's jaws and the variations in the treatment set-up. Consequently there is a risk for tumor recurrence if the target volume is underdosed, or for complications if healthy tissues are overdosed. Additional studies are therefore necessary to assess the effect of these factors on the dose distribution in the junction region and to validate the technique in clinical practice.

The use of electron beams in radiotherapy treatments is less widespread compared to photon beam treatments. In medical linear accelerators, the primary particles used are electrons. If the target used to generate the X-rays by bombarding the material with electrons and the accompanying flattening filter are removed, a high energy electron beam is obtained. A scattering foil is inserted into the beam to scatter the pencil beam and to cover the field size required for electron treatment. The electron beam dose distribution in tissue is however quite different from that of a photon beam. It is characterized by a relatively high surface dose of about $80 \%-90 \%$ of the maximum dose, a flat region around the maximum dose followed by a rapid dose fall of beyond the depth of dose maximum and a low dose tail which actually represents the X-ray contamination of the electron beam, as illustrated in figure $1 \mathrm{~b}$. Because of the higher skin dose and rapid dose fall off at depth, electron beams are often used to treat superficially located tumours. In contrast to photon beams, where generally a multiple beam technique is used, electron beams are mostly delivered by using a single field technique. 

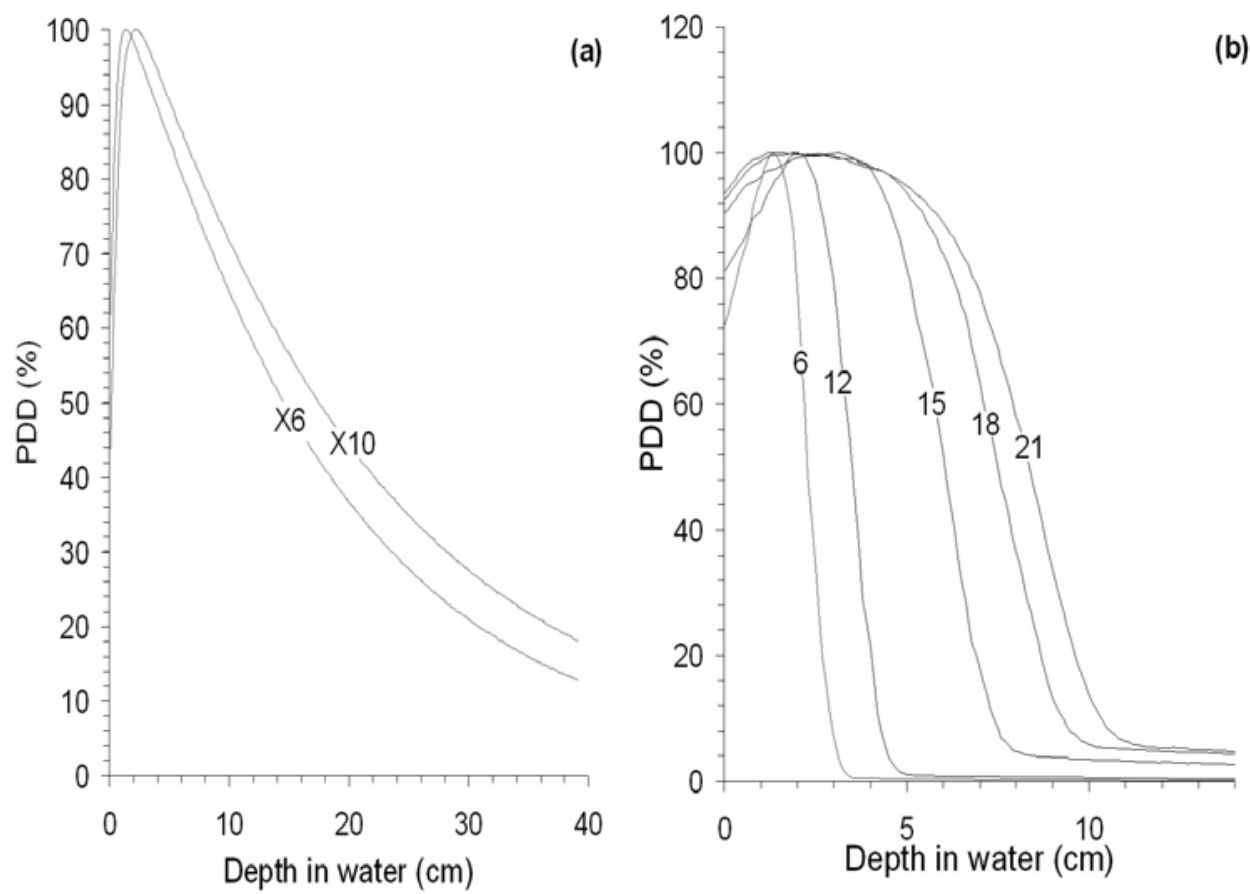

Figure 1: Central axis depth dose curves in water for a 10x10 $\mathrm{cm}^{2}$ field size (a) 6 and $10 \mathrm{MV}$ photon beams for an SSD of $90 \mathrm{~cm}$ and (b) electron beams with energies of 6, 12, 15, 18 and 21 $\mathrm{MeV}$ for an SSD of $90 \mathrm{~cm}$.

In many radiotherapy centers, electron beam treatments are frequently administered based on clinical setup, without formal treatment planning. The dose distribution in an electron beam treatment is, however, frequently very inhomogeneous due to irregular outer surface of a patient, air cavities in or surrounding the treatment volume, or due to oblique incidence of the beam. The dose distribution is, as a result of these factors, difficult to determine without a proper dose calculation algorithm. Even when a TPS is used, dose calculations may not be very accurate in areas having tissue inhomogeneities. To overcome random and systematic errors, additional QA during treatment is important, to prevent the patient from unacceptable high doses in the treatment area or for areas that are under-dosed with the risk for tumor recurrence.

Of all patients treated with radiotherapy, about $5 \%$ to $10 \%$ are candidates for brachytherapy. Brachytherapy is a very local irradiation. The dose is delivered by one or several sealed radioactive sources placed in or adjacent to tumor tissue, having the advantage of highly conforming the dose to the tumor volume and better sparing of the surrounding tissues. As a consequence, higher tumor doses can be achieved. Permanent seed implantation and temporary source loading are important treatment 
options used in brachytherapy. In permanent brachytherapy seeds with low energy photon emitting radionuclides, such as iodine-125 (125) or palladium-103 ${ }^{103 P d)}$, are implanted in tissue using needles that are loaded with the radioactive seeds. The needle or implant device is then removed, leaving the radioactive seeds behind. The seeds remain in the patient's body for the lifetime of the patient, and give off low doses of radiation for weeks or months. Radiation from the seeds travels a very short distance, so the seeds can deliver a very large amount of radiation to a very small area. This decreases the amount of damage produced in healthy tissues that are close to the prostate. The dose is delivered over the lifetime of the sources and the sources undergo complete decay. In temporary brachytherapy, sources are implanted for a specific time duration. The majority of temporary implants are performed with iridium$192\left({ }^{192} / \mathrm{r}\right)$ and cesium-137 (137Cs). The sources are placed in catheters, needles, or other applicators for a brief period of time and then removed when the prescribed dose is delivered. Generally, several treatments are given, and the radioactive substance is removed each time. The treatments are given over a couple of days or weeks. After the last treatment the catheters are removed. To reduce treatment time, high dose rate (HDR) sources are used. The effective transmission in water of both LDR and HDR point sources is presented in figure 2. At points at a distance from the source, the dose fall off is determined by the inverse square law and attenuation, i.e. the combined effect of scattering and absorption of the emitted radiation. For ${ }^{125}$ the attenuation plays a significant role. For ${ }^{60} \mathrm{Co},{ }^{226} \mathrm{Ra},{ }^{137} \mathrm{Cs},{ }^{198} \mathrm{Au}$ and ${ }^{192} \mathrm{Ir}$ the scatter largely compensates for the absorption within the clinical range of a few $\mathrm{cm}$ from the source.

Permanent seed placement is commonly used for early stage prostate cancer, where in addition to radical prostatectomy, Transperineal Interstitial Permanent Prostate Brachytherapy (TIPPB) is an important treatment option. In this treatment the urethra is the organ at risk (OAR). The $D_{10}$ (the dose that covers $10 \%$ volume of the urethra) should be less than $150 \%$ of the prescription dose, the $D_{30}$ less than $130 \%$ and the generally accepted maximum tolerable dose level of the urethra should be restricted to a maximum of 200 gray $(G y)^{3}$. Uncertainties in definition of the urthra on the ultrasound images or in seed positioning during the implantation procedure, errors in needle positioning or deviations in seed activity will influence the dose deposition in this organ, with the risk of too high doses in this organ at risk, resulting in urinary complications ${ }^{4}$. Additional QA, to maximize the probability that each individual treatment is administered consistently, accurately and safely and to evaluate the dose in the OAR, is therefore highly recommended. 


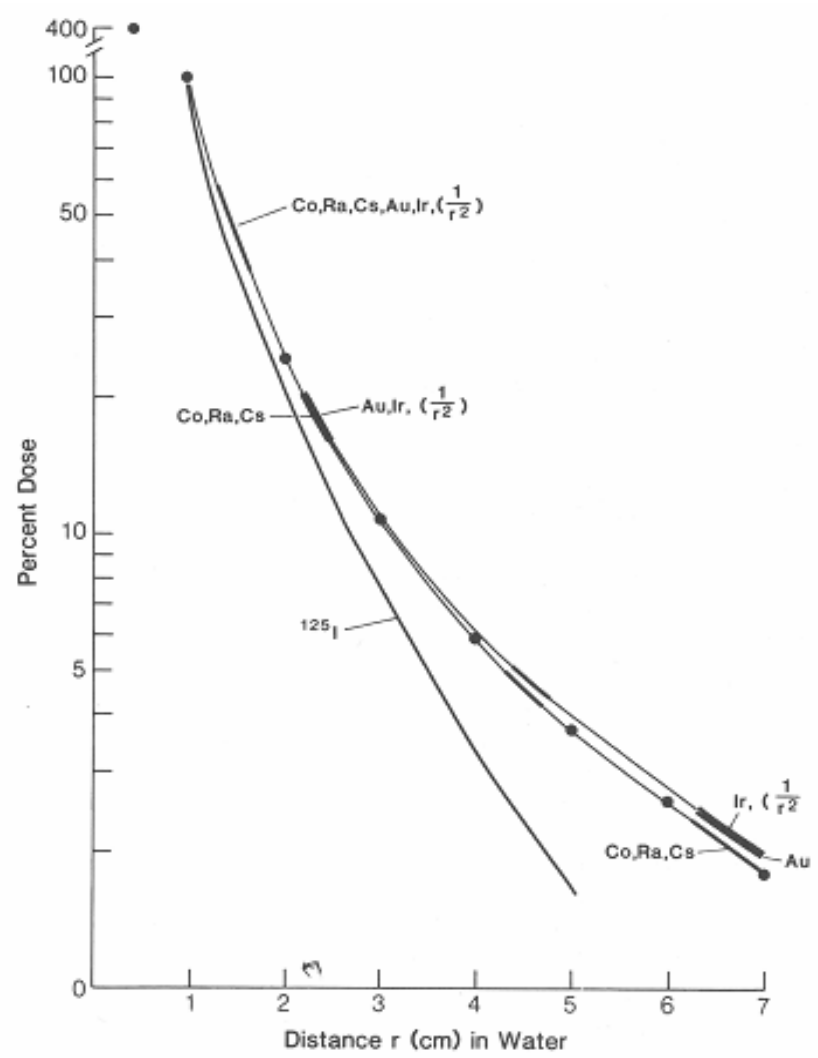

Figure 2: Percent dose variation with distance in water for point sources of ${ }^{60} \mathrm{Co},{ }^{226} \mathrm{Ra},{ }^{137} \mathrm{Cs}$, ${ }^{198} \mathrm{Au},{ }^{192} / \mathrm{r}$ and ${ }^{125}$. Function $\left(1 / \mathrm{r}^{2}\right)$ represents inverse square fall off.

\subsection{IN VIVO DOSIMETRY IN RADIOTHERAPY}

Radiotherapy is a complex process involving many steps before the actual treatment begins. The process starts with the diagnosis and the decision to treat the patient with ionizing radiation. Next a complex simulation and dose calculation procedure starts to arrive at the optimal treatment plan for a particular patient to be given in one or an extended set of fractions (figure 3). Each step in this treatment chain has one or more sources of error and it is therefore important that each single step is executed with the greatest accuracy possible. The uncertainty in each step may influence the accuracy of subsequent steps and, therefore impact the overall treatment result.

Patient dose verification, in vivo dosimetry, serves as an important part of a QA programme in radiotherapy and has been recommended for quality improvement of patient care in radiation therapy by several organizations such as the American 
Association of Physicists in Medicine ${ }^{5}$, and the European Society of Therapeutic Radiology and Oncology 6 . In vivo dosimetry is a tool to measure the radiation dose delivered to patients during radiotherapy. The aim of in vivo dosimetry is to compare these dose measurements with the dose values specified by the radiation oncologist and the dose values calculated by the TPS, to ensure that the accuracy of the prescribed dose to the target volume is within acceptable limits. In addition to the use of in vivo dosimetry for assessing the dose delivered to an individual patient for the detection of various types of errors in the dose delivery process, it can also serve as a tool to verify new treatment techniques in the clinic, after the initial validation of the procedure using phantoms, or as an indicator to assess the dose in organs at risk.

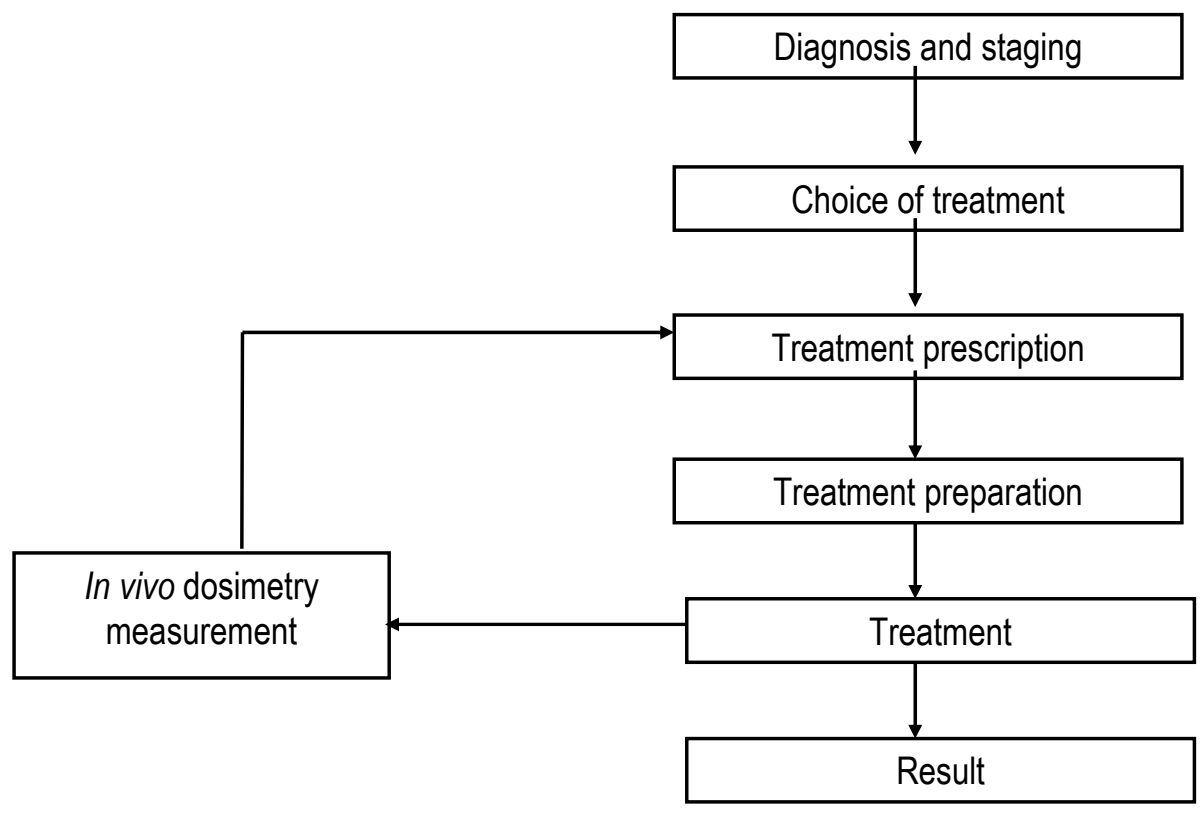

Figure 3: Treatment chain of a radiotherapy treatment.

\subsubsection{In vivo dosimetry as part of a routine QA programme}

There are a lot of control mechanisms to evaluate the accuracy of the single steps in the treatment procedure. These checks are based on QA of the treatment equipment, including the linac and treatment planning system, and on the data transfer among the various types of equipment, such as transfer of data from a CT-scanner to the TPS and from the TPS to the linear accelerator. However, also human procedures are subject to error and determine the accuracy of the actual patient treatment. In practice, despite these control mechanisms random or systematic errors still may occur7, 8. The dosimetric impact of these errors is unique for each case and depends on the 
proportion of fields in error and volume mistreated 7 . An additional check during the actual treatment delivery provides information about the actual dose delivery and gives the ability to correct the dose before the next treatment fraction, when dose errors occur.

\subsubsection{In vivo dosimetry as a tool to validate a treatment technique}

Techniques in radiotherapy are improving very fast. The development of a new treatment technique or an improvement of the actual treatment procedure is a complex procedure. Several steps are required performed by a variety of staff, varying from the radiation therapy technologist and the physicist to the medical doctor. When implementing a new technique, generally phantom measurements are performed. Depending on the extent of the alteration, the accuracy of each single step of the new treatment procedure should be as high as possible and the uncertainty within prescribed limits. Furthermore, the uncertainty of the final dose delivery should be within a pre-defined level. Finally, the new procedure has to be described in a practical guideline in order to ensure that each worker uses the same and correct treatment steps in the entire treatment procedure, and to ensure that the actual treatment of each patient is conform predefined guideliness. Supplementary to a well-defined validation of the new treatment procedure, a final check in clinical practice is essential to determine the accuracy of the new treatment technique during the actual patient treatment and to link the delivered 3D dose distribution to patient related factors.

\subsubsection{In vivo dosimetry to check the dose in organs at risk}

The primary objective of radiation oncology is to make the quality of life for the patient during and after the radiotherapy treatment as high as reasonably achievable. For curative radiotherapy, it is important to deliver a high radiation dose to the tumor, to destroy all cancer cells, thereby limiting the dose to normal tissue, to avoid serious complications during and after the treatment. A high accuracy of the actual treatment is important to provide the desired tumor control rates and the limited dose values at the OARs. In practice, several uncertainties may influence the accuracy of the radiation dose to the target and the OAR, such as uncertainties in the delineation of the regions of interest, in treatment planning related factors, in accelerator performance or seed activity related factors, and errors in the treatment procedure. In vivo dosimetry gives the ability to check the dose in or close to OARs to reduce side effects of a treatment originating from too high doses in a specific organ. The obtained dose information gives the opportunity to adapt the dose or improve the treatment technique. Furthermore, knowing the in vivo dose in OARs is essential to link clinical side effects to absorbed dose values in these organs. 


\subsection{OBJECTIVES AND OUTLINE OF THE THESIS}

In 1994 the first publications concerning a new type of dosimeter, the Metal-Oxide Semiconductor Field Effect Transistor (MOSFET) were published, demonstrating that a MOSFET is a promising new detector in the area of in vivo dosimetry 9,10 . After these initial publications, other publications followed, mostly concerning the properties of the detector in radiotherapy applications ${ }^{11-14}$.

In January 2001, MAASTRO CLINIC defined their wishes to extend the QA programme in treatment delivery with the goal to set-up a broad dose verification programme including a dosimetry check in all patient treatments. Our long term perception for photon beam in vivo dosimetry was based on using Electronic Portal Imaging Devices (EPIDs). EPIDs measure transit dose values, this 2D dose information, can be used for in vivo dosimetry. The use of the EPID for in vivo dosimetry was, however, very new at that time and the clinical use of the system could not be obtained within our defined deadline. To achieve our short time in vivo dosimetry goals in photon beams, combined with our aim to perform in vivo dose measurements at extended distance, in the surface region, in electron beams and for brachytherapy purposes, a point detector was selected. The MOSFET dosimeter, a relatively new dosimeter in the range of detectors suitable for in vivo dosimetry in radiotherapy, seemed a suitable detector for our range of applications. The selection was primarily based on the broad spectrum of applications where the MOSFET detector was supposed to be useful as a clinical dosimeter. A secondary advantage of the MOSFET system was the simple dose readout method and the lack of correction factors. Furthermore, the portability of the system with the opportunity to perform dose measurements at several linacs using one dose verification system, if necessary combined with an additional set of dosimeters with accompanying bias supply, was a major advantage in order to reduce costs. The simplicity of the system to implement the dose measurement and to integrate the measurement protocol into the work processes were other important factors.

The MOSFET dosimeter is a miniature non-intrusive semiconductor radiation detector. The basic MOSFET structure is depicted in figure 4a. The MOSFET is a sandwich type device with a positively doped (P-type) silicon semiconductor substrate in addition to an insulating oxide layer separating it from the negatively doped ( $\mathrm{N}$-type) silicon. Prior to radiation a sufficiently large negative voltage is applied to the poly-silicon gate, with the result that a significant number of minority carriers (holes) will be attracted to the oxide-silicon surface from both the bulk silicon substrate and the source and drain regions ${ }^{9}$. Once a sufficient concentration of holes has accumulated there, a conduction channel is formed, allowing current to flow between the source and drain. The voltage necessary to initiate current flow is known as the device threshold voltage. When a MOSFET device is irradiated, three things happen within the silicon dioxide layer (sensitive region): a build-up of trapped charge in the oxide; the increase in the number of interface traps; and the increase in the number of bulk oxide traps. Electron-hole 
pairs are generated within the silicon dioxide by the incident radiation. Electrons move out of the gate electrode while holes move in a stochastic fashion towards the $\mathrm{Si} / \mathrm{SiO}_{2}$ interface where they become trapped in long term sites, causing a negative threshold voltage shift, which can persist for years. The difference in voltage shift before and after exposure can be measured, and is proportional to dose (figure $4 \mathrm{~b}$ ) 9 .

The aim of the studies described in this thesis is to investigate the use of MOSFET dosimeters in various areas of radiotherapy. The validation of the detector, the phantom measurements to test the measurement procedure, the results of patient measurements, and methods for the implementation of in vivo dosimetry in daily practice for both internal and external beam radiotherapy are described, with the goal to: (1) detect various types of errors in the dose delivery process, (2) to validate new treatment techniques in the clinic and (3) to assess the dose to organs at risk.
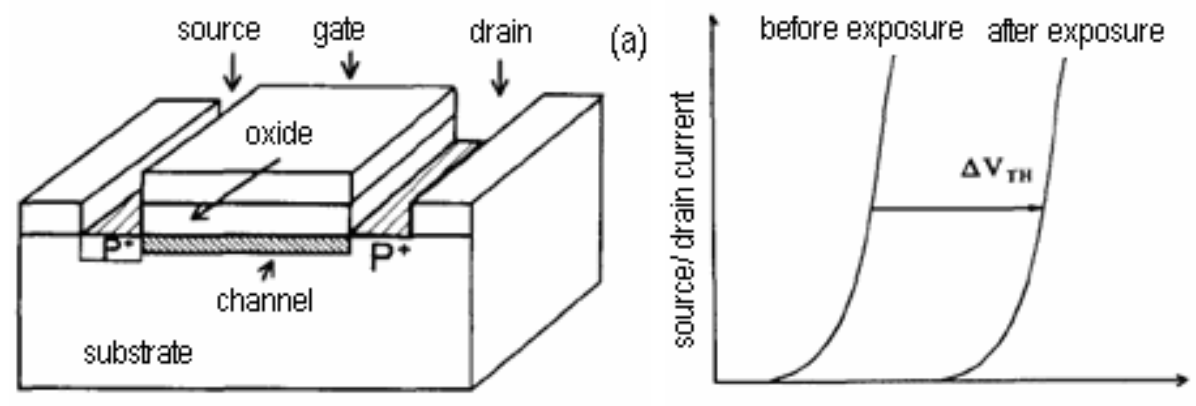

(b)

Figure 4a: The basic MOSFET structure, showing the oxide, $\mathrm{SiO}_{2}$, the substrate, Si, the source and the drain and b: gate voltage before and after exposure as a function of source/ drain current.

In chapters 2 and 4 results are presented of in vivo dose measurements performed to validate new treatment techniques in clinical practice, whereas in chapter 3 the use of MOSFETs for verifying the existing practice of applying electron beams is elucidated.

In chapter 2 the first goal was to describe the accuracy of the MOSFET detector for dose measurements in a junction field technique, using half beams. The second goal was to evaluate the dose across the split line. The accuracy of the detector was determined by an extended set of phantom measurements and the results of this phantom study were used for patient measurements, performed on 5 patients receiving loco-regional treatment for breast cancer, during 10 sequential treatment fractions. The influence of factors related to mechanical properties and QC procedures of the linear accelerator are also discussed.

In chapter 3, we describe the use of MOSFET dosimeters for entrance in vivo dose verification during electron beam treatment, performed without build-up material on the detector to prevent the target volume from shadowing effects by the detector. A 
comprehensive set of phantom measurements was performed to determine factors to convert the surface readings to absolute dose values at depth of dose maximum. Patient measurements were performed to evaluate the detectors under clinical conditions and to test the in vivo procedure in daily practice.

The first aim of the study, described in chapter 4 , was to verify a new dose calculation technique, a 3-dimensional (3D)-dose calculation for TBI, with the goal to optimize this treatment by using $3 \mathrm{D}$ treatment planning for individual patients. A set of phantom measurements was performed, to check the accuracy of a commercial TPS under TBI conditions. The second aim of this study was to use MOSFET detectors for in vivo dose verification of the actual delivered dose and to compare these values with the 3Ddose distribution calculated for our TBI technique by a commercial TPS, and with measured TLD results.

In chapters 5 and 6, the application of MOSFET dosimetry for dose verification in organs at risk during brachytherapy is described. In chapter 5, a new linear 5MOSFET-array dosimeter was validated for in vivo dose verification after permanent prostate implantation using ${ }^{125}$. Patient measurements were performed in order to provide information about the actual dose in the urethra. The results were used to evaluate the implantation technique, including the accuracy of the TPS and will be used in the future to link dose distributions to OARs and clinical side effects.

The goal of the study described in chapter 6 was to investigate the feasibility of a linear-MOSFET-array for real-time dosimetry during permanent prostate brachytherapy with the opportunity to adapt the treatment plan if the dose in the urethra would be too high. The results were used to check the treatment chain and prevent patients from getting urinary complications as a consequence of too high doses in this organ at risk.

Chapter 7 discusses the results of the studies described in this thesis, their clinical relevance and their implementation in clinical practice. Furthermore future developments are presented in this chapter. 


\section{REFERENCES}

1. Ferlay J, Autier $\mathrm{P}$, Boniol M, et al. Estimates of the cancer incidence and mortality in Europe in 2006. Ann Oncol 2007;18:581-592.

2. Van Dyk J, J. M. Galvin, G. P. Glasgow, E. B. Podgorsak. Physical aspects of total and half body irradiation. Report of Task Group 29, American Association of Physicists in Medicine (AAPM). 1986.

3. Salembier C, Lavagnini P, Nickers $P$, et al. Tumour and target volumes in permanent prostate brachytherapy: a supplement to the ESTRO/EAU/EORTC recommendations on prostate brachytherapy. Radiother Oncol 2007;83:3-10.

4. Locke J, Ellis W, Wallner K, et al. Risk factors for acute urinary retention requiring temporary intermittent catheterization after prostate brachytherapy: a prospective study. Int J Radiat Oncol Biol Phys 2002;52:712-719.

5. Kutcher GJ, Coia L, Gillin M, et al. Comprehensive QA for radiation oncology: report of AAPM Radiation Therapy Committee Task Group 40. Med Phys 1994;21:581-618.

6. Ferreira IH, Dutreix A, Bridier A, et al. The ESTRO-QUALity assurance network (EQUAL). Radiother Oncol 2000;55:273-284.

7. Klein EE, Drzymala RE, Purdy JA, et al. Errors in radiation oncology: a study in pathways and dosimetric impact. J Appl Clin Med Phys 2005;6:81-94.

8. Noel A, Aletti $P$, Bey $P$, et al. Detection of errors in individual patients in radiotherapy by systematic in vivo dosimetry. Radiother Oncol 1995;34:144-151.

9. Soubra M, Cygler J, Mackay G. Evaluation of a dual bias dual metal oxide-silicon semiconductor field effect transistor detector as radiation dosimeter. Med Phys 1994;21:567-572.

10. Gladstone DJ, Lu XQ, Humm JL, et al. A miniature MOSFET radiation dosimeter probe. Med Phys 1994;21:1721-1728.

11. Scalchi P, Francescon P. Calibration of a MOSFET detection system for 6-MV in vivo dosimetry. Int J Radiat Oncol Biol Phys 1998;40:987-993.

12. Ramani R, Russell S, O'Brien P. Clinical dosimetry using MOSFETs. Int J Radiat Oncol Biol Phys 1997;37:959-964.

13. Butson MJ, Rozenfeld A, Mathur JN, et al. A new radiotherapy surface dose detector:the MOSFET. Med Phys 1996;23:655-658.

14. Gladstone DJ, Chin LM. Real-time, in vivo measurement of radiation dose during radioimmunotherapy in mice using a miniature MOSFET dosimeter probe. Radiat Res 1995;141:330-335. 




\title{
CHAPTER
}

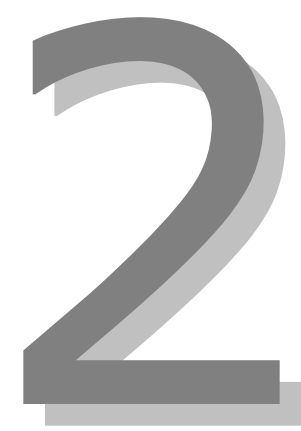

\section{Clinical dosimetry with MOSFET dosimeters to determine the dose along the field junction in a split beam technique}

RADIOTHERAPY AND ONCOLOGY 67 (2003) 351-357

\author{
Esther J. Bloemen-van Gurp \\ Wim F.J. du Bois \\ Peter A. Visser \\ laïn Bruinvis \\ Derk Jalink \\ Joep Hermans \\ Philippe Lambin
}




\section{ABSTRACT}

Background and purpose: We report on the accuracy of MOSFET detectors to measure dose distributions in the region of a field junction in a split beam technique, compared to ionisation chamber and photographic film. We present a study on 5 patients receiving loco-regional treatment for breast cancer.

Materials and methods: The dose variation at the junction was measured with the Patient Dose Verification System Model TN-RD-50 (MOSFET system). Phantom measurements were performed to investigate the MOSFET accuracy in a half field matching method and the influence of factors related to the accelerator. The aim of the patient measurements was to determine the effect of patient related factors on the dose at the junction over a period of 10 irradiation fractions.

Results: The MOSFET detector overestimates the dose in the junction area, therefore a correction factor was determined to correct the MOSFET results. Phantom measurements showed overdosages as well as underdosages, depending on the matching field direction ( $\mathrm{X}$ or $\mathrm{Y}$ ). Patient measurements showed dose values that deviated up to $133 \% \pm 3 \%$ (2SD). The average values of 10 irradiation sessions showed a continuous, almost linear, variation of dose value across the junction.

Conclusions: (1) Significant dose deviations in the junction area average out over repeated treatments; (2) One individual measurement in the junction area should not lead to any action; (3) A linac QA procedure to check the dose at the junction line should simulate the clinical situation sufficiently; (4) MOSFET dosimeters overestimate dose values in the penumbra region. 


\subsection{INTRODUCTION}

Adjacent treatment fields are commonly employed in external beam therapy. There are several reasons why a treatment volume is treated with adjacent fields; an important one is the extension of the treatment volume. If the treatment volume is longer than the maximum field size, more than one beam has to be used (e.g. irradiation of the entire muscle compartment in soft-tissue sarcoma). Another reason may be the anatomy of the patient: the patient's contour and normal tissue constraints may require more than one beam configuration (e.g. irradiation of head and neck tumours when lateral neck fields are placed adjacent to an anterior supraclavicular field).

A number of techniques have been developed to achieve dose uniformity in the area of the field junction. One technique uses split beams. With this method, the beam is split along the plane containing the central axis by using a half-beam block, thus removing the geometric divergence of the beams at the split line. The split beam technique, using asymmetric collimator jaws, is easy to perform and reduces the treatment time significantly. In theory, the split beams should match perfectly. In the clinical situation however, there is a possibility of introducing dose deviations across the junction. These can originate from factors related to mechanical properties and quality assurance (QA) procedures of the linear accelerator's jaws and to the treatment set-up, e.g. patient movements. Consequently, this region is at risk for tumour recurrence if it is underdosed or for complications if it is overdosed.

To determine the dose across the split line, in vivo dosimetry may be used. Various techniques have been employed, e.g. TLD ${ }^{10,17}$, standard film ${ }^{1,2,9,13}$, gafchromic film ${ }^{14}$ and semiconductors $2,3,5,13$. Recently there has been an increasing interest in the use of Metal Oxide-silicon Semiconductor Field Effect Transistors (MOSFETs) as a device for in vivo dosimetry7, 8. Since January 2001, our institute has applied MOSFET dosimetry as part of a quality assurance programme for treatment delivery. In the present study we investigated the use of such detectors to measure dose distributions in the region of a field junction in a split beam technique.

In this paper we report on the accuracy of the MOSFET detector in such an application, compared to ionisation chamber and photographic film and we present a study on 5 patients receiving loco-regional treatment for breast cancer. This loco-regional technique consists of three half-fields, using split beams. A dose of 50 Gy in 25 fractions is delivered. The beams are split along the plane containing the central axis by closing the diaphragms or leaves, thus removing the geometric divergence (fig. 1). Measurements were performed at the centre of the junction between the upper supraclavicular and the lower breast fields. The influence of factors related to the mechanical properties and QA procedures of the linear accelerator, are also dicussed. 

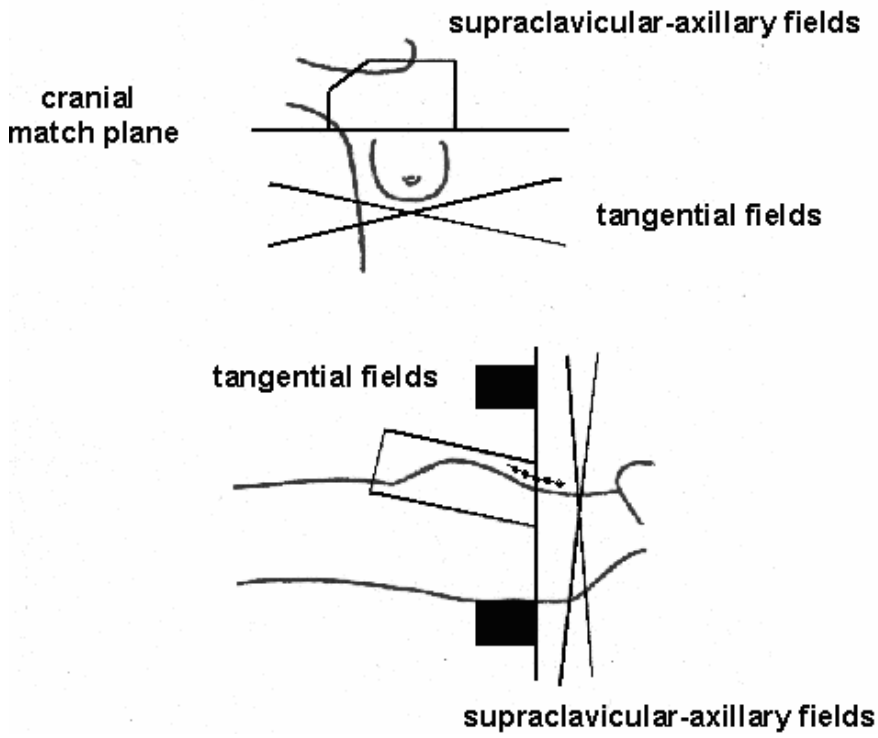

Figure 1: Loco regional radiation technique. Three matching fields are employed in the breast cancer treatment technique; a: anterior view of the treatment technique; $b$ : lateral view of the treatment technique.

\subsection{METHODS AND MATERIALS}

\subsubsection{Linear accelerator}

Measurements were performed using an Elekta SLi15 linear accelerator with a multileaf collimator (MLC). The MLC consists of 40 pairs of leaves, each projecting to a width of $1 \mathrm{~cm}$ at the isocenter (100 cm source-axis- distance). The leaves are made of tungsten and are $7 \mathrm{~cm}$ thick. The leaves replace the $\mathrm{Y}$ - blocks, while the X-blocks are standard. Each leaf can travel $12.5 \mathrm{~cm}$ across the central beam axis (collimator rotation axis) and has a positional accuracy of $1 \mathrm{~mm}$. The end of each leaf is rounded which causes a broader penumbra than with focussed collimator blocks. The entire MLC assembly fits inside the standard treatment head, providing shaping for the full $40 \times 40$ $\mathrm{cm}^{2}$ treatment field 6,11 .

\subsubsection{Dosimeter system}

To determine the dose across the junction, the patient Dose Verification model TN-RD50 (MOSFET system), produced by Thompson and Nielsen Electronics Ltd. (Ottawa, Canada), was used. The MOSFET dosimeter is an electronic device, which integrates the radiation dose. A silicon chip of $1 \mathrm{~mm} \times 1 \mathrm{~mm}$ (active area $0.2 \mathrm{~mm} \times 0.2 \mathrm{~mm}$ ) is 
attached to a $1 \mathrm{~mm}$ layer of black epoxy at the end of a flexible cable. For high-energy photon beams (above $1 \mathrm{MV}$ ) the flat side of the dosimeter is facing the beam. The water equivalent depth of the MOSFET is about $0.8 \mathrm{~mm}$ or $1.8 \mathrm{~mm}$, depending on which sensor side faces the beam ${ }^{15}$. In this study the detectors were placed with the round side of the bulb in contact with the surface of the patient, so measurements were performed at an equivalent depth of $0.8 \mathrm{~mm}$. The MOSFET dosimeter is sensitive to radiation damage and one of its electrical properties ("threshold voltage") changes continuously with the absorption of radiation. This voltage is a function of absorbed dose. The signal can be permanently stored. Variation in signal with temperature changes is within the detector's reproducibility ${ }^{15,}{ }^{16}$. A negligible variation of the dosimeter response was determined for gantry angles up to 25 degrees. For angles between 25 to 90 degrees, the response decreased up to 2.5\%15. Dose- rate independency, as stated in literature ${ }^{7}$, was checked. To simulate variation in dose per pulse, measurements were performed with MOSFETs and an ionisation chamber at 80,100 and $140 \mathrm{~cm}$ from the focus using $6 \mathrm{MV}$ photon beams. This resulted in relative dose per pulse values of 1.6, 1.0 and 0.5 . The standard deviation for the measured ratios of the two signals was within $1.5 \%$.

A standard bias supply (TN-RD-20), equipped with a $9 \mathrm{~V}$ battery, provided a regulated bias voltage of $7.4 \mathrm{~V}$ to the standard dosimeters (TN502), with a sensitivity of 1 $\mathrm{mV} / \mathrm{cGy}$. It also provided the interface from dosimeter to reader; this connection is necessary during "zero-ing" and "reading" the dosimeters. The total threshold voltage was read out in $\mathrm{mV}$ and converted to the corresponding dose in cGy.

Calibration was performed under full build-up, with the $6 \mathrm{MV}$ photon beam and a cylindrical ionisation chamber (PTW type 31002, Physikalisch Technische Werkstaetten, Freiburg) as a reference. The calibration factors for the individual dosimeters ranged from 1.05 to 1.10 . The reproducibility of these factors is about \pm 3 $\%(2 \mathrm{SD})$ at about $100 \mathrm{mV}$ voltage shift 15,16 .

The measurements were divided into two parts: phantom measurements and patient measurements.

\subsubsection{Phantom measurements}

All phantom measurements described below, were performed with $6 \mathrm{MV}$ photon beams, using a rectangular polystyrene phantom $(30 \mathrm{~cm} \times 30 \mathrm{~cm} \times 15 \mathrm{~cm})$ at $100 \mathrm{~cm}$ SSD and $0^{\circ}$ gantry angle. Five dosimeters were placed over a length of $1.4 \mathrm{~cm}$, with 1 $\mathrm{mm}$ distance between them. Two half-blocked, $10 \mathrm{~cm} \times 10 \mathrm{~cm}$, adjacent fields using collimator angles of 0,90 and 270 degrees were delivered. Dosimeters 1 and 2 were placed at the superior border of the inferior fields, dosimeter 3 exactly on the match line and dosimeters 4 and 5 at the inferior border of the superior field. The detectors were covered with $1.5 \mathrm{~cm}$ of a water-equivalent build-up material. The fields were set-up using laser lines. The central beam axis was positioned at the centre of the phantom 
and the automated field set-up system was used; i.e. collimator angle and jaws were set without moving the treatment table.

The dose variation in the match region was evaluated in the following way; a straight line was fitted through a plot of the readings of dosimeter 1, 2, 4 and 5. In case of an ideal match the plot of the reading of dosimeter 3 was expected to coincide with this line (within 1 SD). Over- and underdosage were defined as the ratio of the dosimeter 3 reading and the mean value of dosimeter $1,2,4$ and 5 readings.

The aim of the phantom measurements was to investigate the MOSFET accuracy in a half field matching method (2.3.1), the influence of factors related to the accelerator (2.3.2) and the effect of contaminant electrons on the patient measurements (2.3.3). The results of these phantom measurements were used to determine the matching characteristics under optimal conditions, without deviations caused by patient movements.

\subsubsection{MOSFET accuracy in a half field matching method}

To determine the accuracy of the MOSFET detector in the junction area, comparisons with film and ionisation chamber were made. Kodak-Xomat $\mathrm{V}$ films were positioned under $1.5 \mathrm{~cm}$ of tissue equivalent build-up material on a polystyrene phantom and densities were readout with a Multidata model 9710 densitometer (MultiData, St-Louis). A PTW type 31002 cylindrical ionisation chamber was scanned across the junction at $1.5 \mathrm{~cm}$ depth in water, with the chamber's axis positioned parallel to the junction. The Semi Flex Tube Chamber has a diameter of $0.7 \mathrm{~cm}$ and a volume of $0.125 \mathrm{~cm}^{3}$. Five repeated sets of measurements were performed and the results were averaged.

\subsubsection{The influence of accelerator related factors}

The measurements to investigate the accelerator related factors were divided in two parts; measurements to investigate the accuracy of collimator angle and $X$ - and $Y$ block setting and measurements to determine the reproducibility of block positioning.

The accuracy of collimator angle and block settings was investigated for three situations. In the first situation the $X_{1}$ - and $X_{2}$ - blocks were matched. The same MOSFET set up was then used matching the $X_{2}$ - block with the $Y_{1}$-leaves (situation 2) and matching the $X_{2}$ - block with the $Y_{2}$ - leaves (situation 3). The three situations are described in table 1. For each situation, three sets of measurements were performed and the results were averaged.

The reproducibility of block positioning matching the $X_{1}$ - and $X_{2-}$ blocks, was determined with the ionisation chamber. Five repeated measurements were performed and for each measurement the blocks were positioned using the automated set- up system. 


\begin{tabular}{lcccccc} 
Field & \multicolumn{2}{c}{ situation 1 } & \multicolumn{2}{c}{ situation 2} & \multicolumn{2}{c}{ situation 3} \\
Field size & AP inferior & AP superior & AP inferior & AP superior & AP inferior & AP superior \\
$\mathrm{X}_{1}$ & & & & & & \\
$\mathrm{X}_{2}$ & 0.0 & 10.0 & 5.0 & 10.0 & 5.0 & 10.0 \\
$\mathrm{Y}_{1}$ & 10.0 & 0.0 & 5.0 & 0.0 & 5.0 & 0.0 \\
$\mathrm{Y}_{2}$ & 5.0 & 5.0 & 0.0 & 5.0 & 10.0 & 5.0 \\
Gantry & 5.0 & 5.0 & 10.0 & 5.0 & 0.0 & 5.0 \\
Collimator & 0 & 0 & 0 & 0 & 0 & 0 \\
\hline
\end{tabular}

Table 1: Field set-up of phantom measurements investigating the influence of collimator, block and leaf setting.

\subsubsection{The effect of contaminant electrons on the junction dose}

The MOSFET measurements on the patients were performed without any build-up material (see below); therefore the effect of contaminant electrons on the determined over- or underdosage values was investigated. The MOSFETs were covered with 0 , $0.5,1.0$ and $1.5 \mathrm{~cm}$ build-up material, respectively. For all depths five repeated sets of measurements were performed, the results were averaged and the ratios of the dosimeter 3 reading and the mean value of dosimeter 1,2, 4 and 5 readings were computed. The same measurements were performed with the ionisation chamber in water and the average ratios were compared to those of the MOSFETS.

\subsubsection{Patient measurements}

The aim of the patient measurements was to determine the effect of patient related factors, such as patient movement, on the dose at the junction over a period of 10 irradiation fractions. Measurements were performed on 5 patients. Three of them had received a complete mastectomy. Two patients had received breast-conserving surgery. Two half-blocked adjacent fields using $6 \mathrm{MV}$ photon beams were delivered. In one patient a $10 \mathrm{MV}$ beam for the upper supraclavicular field was used (the correction for energy dependence when measuring in a $10 \mathrm{MV}$ beam instead of in a $6 \mathrm{MV}$ beam is less than $1 \%$ ). For all patients, the $X_{2}$ - block of the cranial field matched the $Y_{1}$ - and $\mathrm{Y}_{2}$ - leaves of the caudal fields. The details of field parameters are described in table 2. Five dosimeters were taped on the skin, at the same location during all measurements. The fields were set up in an isocentric technique, using laser lines, and the automated set-up system, without moving the treatment table. Five MOSFETs were placed as described in the first paragraph of 2.3. To determine the precise location of the MOSFET dosimeters during the 10 irradiation fractions, the MOSFET location was indicated on the patient's skin. One person performed all measurements. 
Build-up material on the patient's skin during 10 irradiation fractions would significantly increase the skin dose under the detector and would reduce the dose at depth. This effect is unacceptable over 10 irradiation fractions. Therefore measurements were performed without build-up. The purpose was to compare the readings of the five dosimeters, in order to assess the dose variation over the junction during 10 radiation fractions. Since the conversion to dose is uncertain when no build-up is used, the results are stated as relative readings.

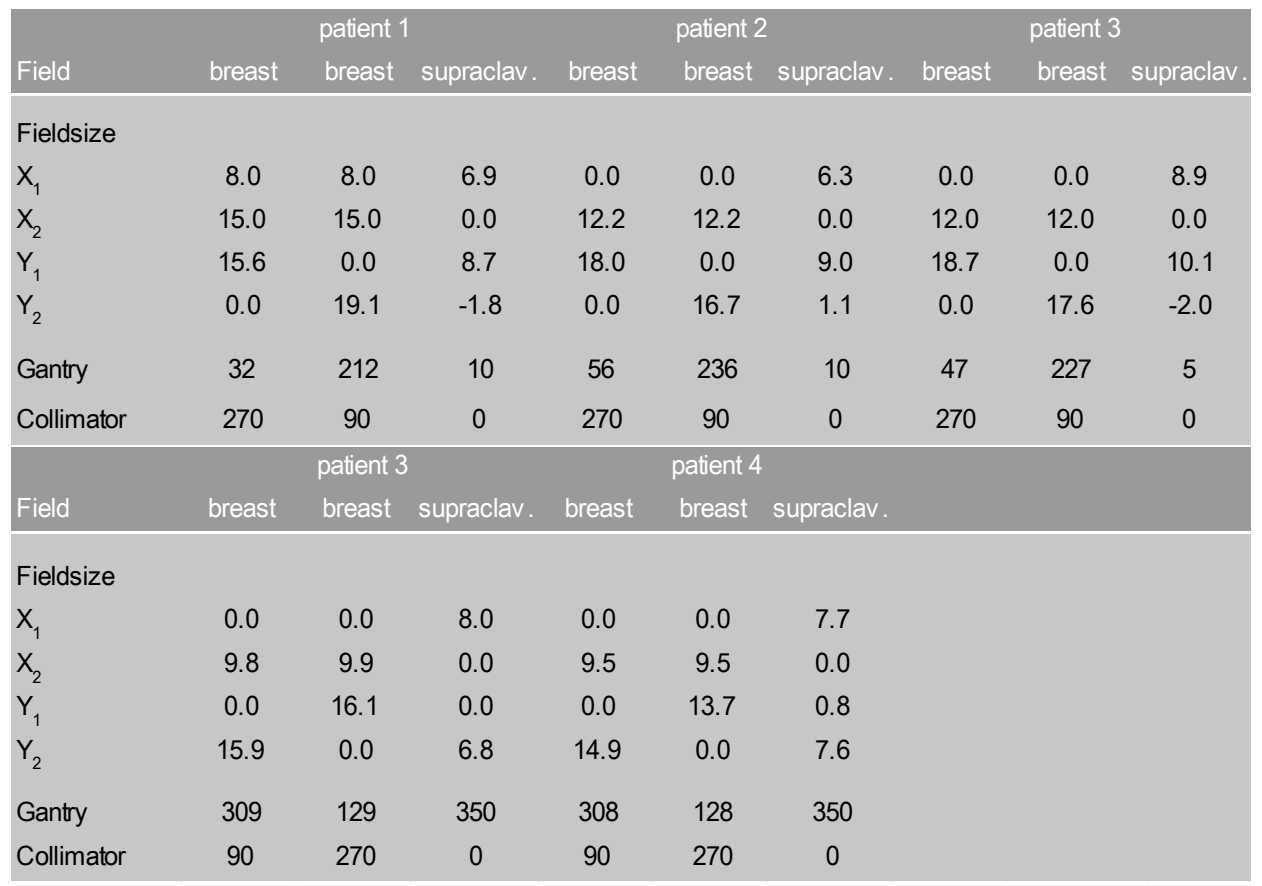

Table 2: Field set-up of patient measurements. 


\subsection{RESULTS}

\subsubsection{Phantom measurements}

\subsubsection{MOSFET accuracy in a half field matching method}

Matching $X_{1}$ - and $X_{2-}$ blocks, the MOSFET measurements showed an overdosage of $111 \% \pm 1.7 \%$ ( $1 \mathrm{SD}$ ). For film measurements this value was $106 \% \pm 2.0 \%$ (1 SD) and for ionisation chamber measurements $105 \% \pm 0.7 \%$ (1 SD). It appears that the MOSFET detector gives higher readings in the penumbra region. Therefore a correction factor was determined to correct the MOSFET results. This factor is equal to the ratio of the ionisation chamber and MOSFET overdose values and has the value of $0.95 \pm 0.02$ ( $1 \mathrm{SD}$ ). Matching $\mathrm{X}_{2}$ - blocks and $Y_{1-}$ - leaves, the MOSFETs showed a relative dose value of $100 \% \pm 2.0 \%$ at the junction area. Film measurements showed an underdose of $95 \% \pm 1.5 \%$ (1 SD). The correction factor matching $X_{2}$ - blocks and $Y_{1}$ - leaves has the value of $0.95 \pm 0.02$ (1SD), equal to the factor matching $X_{1}$ - and $\mathrm{X}_{2}$ - blocks. All measurement results described below are corrected with this factor.

\subsubsection{The influence of factors related to the accelerator}

The measurement results of the three test situations, investigating the accuracy of collimator angle and block and leaf setting, are shown in table 3 . Matching $X_{1}$ - and $X_{2}$ blocks (situation 1) gives an overdose of $105 \% \pm 1.7 \%$ (1 SD). Matching $X_{2^{-}}$block and the $Y_{1}$ - leaves (situation 2) gives an underdose of $95 \% \pm 1.4 \%$ (1 SD) and matching the $X_{2}$ - block and the $Y_{2}$ - leaves (situation 3 ) an underdose of $91 \% \pm 1.7 \%$ $(1 \mathrm{SD})$ at the match line.

The test to investigate the reproducibility of block positioning showed for the $X_{1-}$ and $X_{2}$ - blocks a mean overdose value of $106.7 \%$. The standard deviation was $0.53 \%$ (1 SD). The overdose value ranges between $106.1 \%$ and $107.4 \%$.

\begin{tabular}{lcccccc} 
& \multicolumn{2}{c}{ situation 1 } & \multicolumn{2}{c}{ situation 2} & \multicolumn{2}{c}{ situation 3} \\
dosimeter & mean reading & S.D. & mean reading & S.D. & mean reading & S.D. \\
\hline 1 & 103.6 & 2.12 & 111.2 & 0.00 & 103.5 & 2.12 \\
2 & 107.0 & 2.83 & 107.2 & 0.71 & 101.3 & 0.71 \\
3 & 117.6 & 1.41 & 109.6 & 1.41 & 98.0 & 1.41 \\
4 & 106.0 & 0.71 & 109.2 & 0.71 & 99.0 & 0.00 \\
5 & 107.4 & 2.12 & 109.2 & 2.12 & 102.5 & 3.54
\end{tabular}

Table 3: Results of phantom measurements. For all situations three measurements were performed and the results were averaged. 


\subsubsection{The effect of contaminant electrons on the junction dose}

The ratio of the readings performed at various depths, gives an almost horizontal line over the measuring depths for both MOSFET and ionisation chamber. No demonstrable influence of contaminating electrons on the MOSFET reading at the surface was found.

\subsubsection{Patient measurements}

Fifty measurements were performed, during 10 different irradiation sessions of 5 patients. For all patients, the $X_{2}$ - block of the cranial field matched the $Y_{1}$ - and $Y_{2^{-}}$ leaves of the caudal fields. The phantom measurements in these situations (3.1.1) showed that a correction factor of 0.95 has to be applied. The average of the 10 measurements, corrected for overestimation of the detector, is given in figure 2a-e. A point depicts the mean value for each single detector. The error bars indicate the standard deviation of the measurements. An ideal field match will show a smooth line through the data points.

For patient 2 build-up was used in the inferior tangential breast fields, required for adequate treatment of skin involvement. This caused the relatively high readings of dosimeter 1 and 2 in the caudal region. The build-up influenced the reading of dosimeter 3 up in this caudal region. As expected, the reading of this dosimeter was higher than the reading of dosimeter 3 for the other patients, but was still in a line with the reading of dosimeter 2 and 4 for this patient. We can conclude that there is a smooth dose variation in the measurement area, in spite of the partial build-up in the junction area. Measurements on patient 4 showed low readings of dosimeter 4 and 5 in the superior supraclavicular region. In this region, a $10 \mathrm{MV}$ beam was used. Due to this higher energy (10 MV instead of $6 \mathrm{MV}$ ) there are less contaminating and secondary electrons in the surface region. This explains the lower skin dose, measured with the MOSFET dosimeters in this superior supraclavicular area. The reading of dosimeter 3 was influenced by the use of this $10 \mathrm{MV}$ beam, but was still in a line with the reading of dosimeter 2 and 4 . There was a homogeneous dose in the whole junction area, in spite of the use of two different energies.

The mean results for all patients showed no significant deviations, because of the relative high SD of the measurements in the central junction area. The individual irradiation session measurements showed, in contrast to the mean results, large dose errors across the junction. Errors of up to $133 \% \pm 3.0 \%$ (2SD) were measured. These matching errors, caused by patient movement during radiation, are responsible for the high SD. 

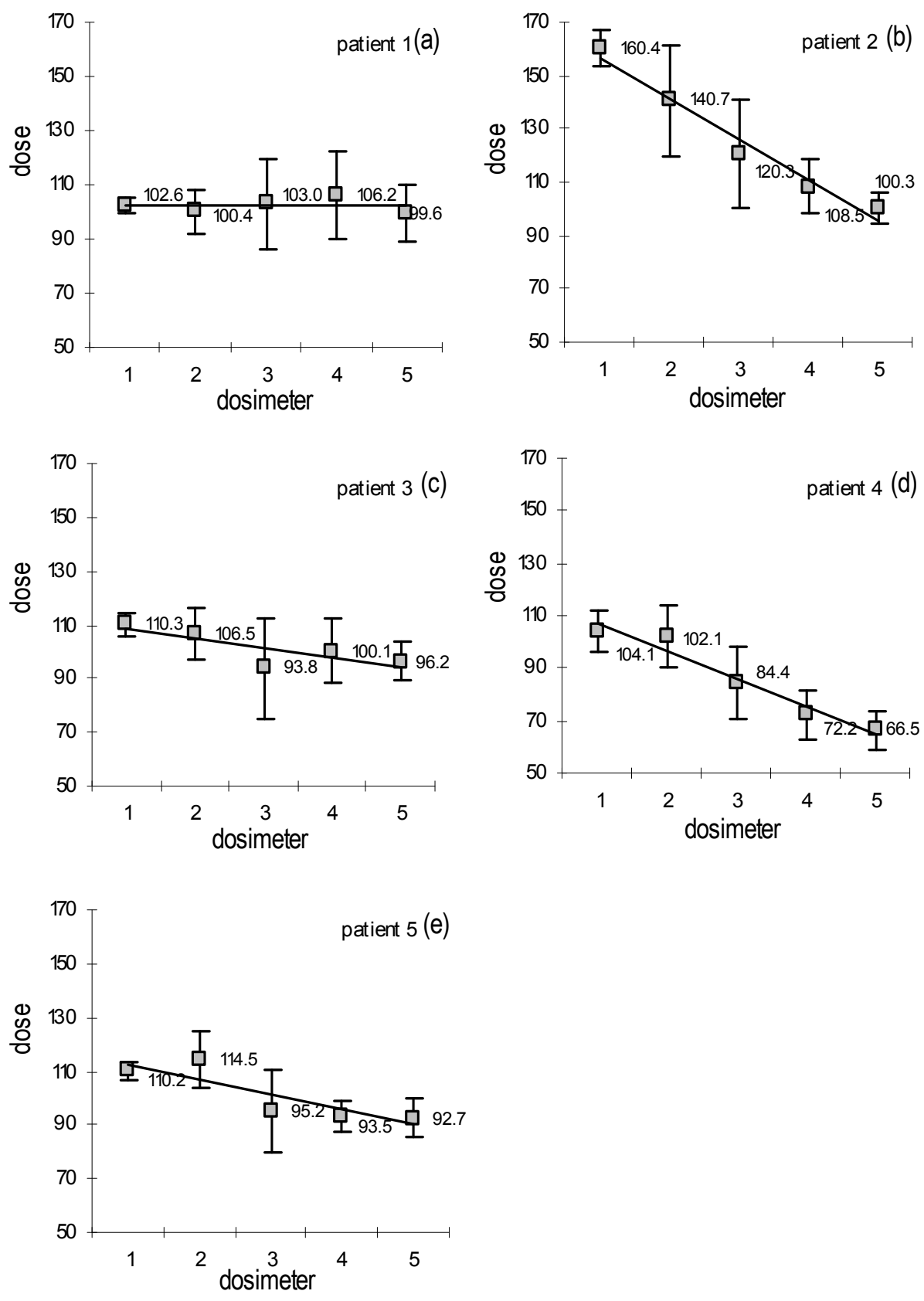

Figure 2a-e: Patient measurements. Average readings of 10 patient measurements for 5 patients. A point depicts the average value for each single detector. The vertical lines indicate the standard deviations of the 10 measurements. 
The standard deviation for the individual MOSFET-dosimeters, during 50 measurements, is illustrated in figure 3. The dosimeter on the junction (3) presents the highest SD. Movements mostly affects the dose at the junction. The smallest standard deviations are seen for dosimeter 1 and 5 .

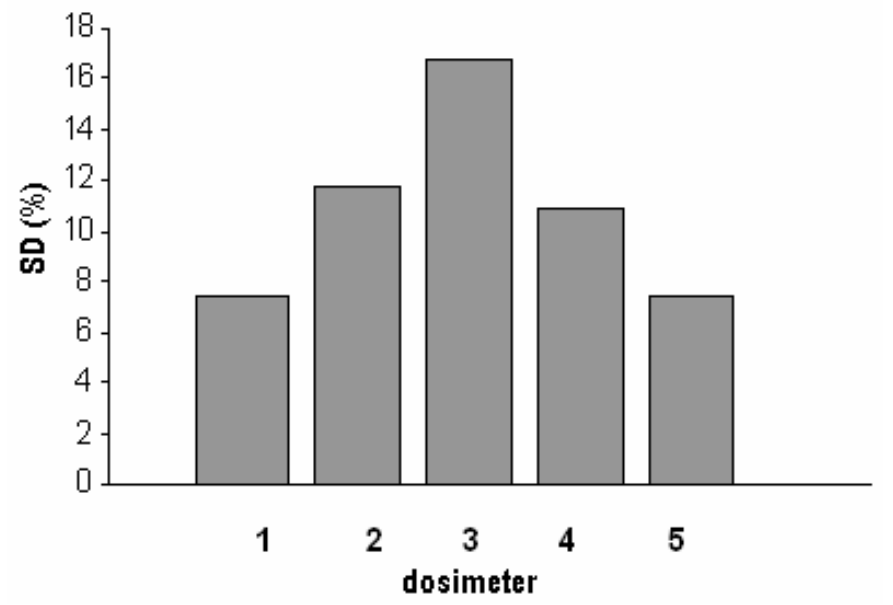

Figure 3: Patient measurements. Standard deviations of the 5 MOSFET readings during 50 patient measurements. Dosimeter 1 and 2 were placed at the border of the inferior tangential breast fields, dosimeter 3 exactly on the match line and dosimeter 4 and 5 at the border of the superior supraclavicular field.

\subsection{DISCUSSION}

Significant dose variation at the junction can be caused by a minimal positioning inaccuracy of the jaws at the position of the central axis (offset). A $10 \%$ deviation of the dose value around the $50 \%$ point on a beam profile is related to a misalignment of $1 \mathrm{~mm}$ at a SSD of $100 \mathrm{~cm}$ for one block or $0.5 \mathrm{~mm}$ per block when the deviation for both blocks is in opposite position. Our QA programme is performed using film dosimetry and a deviation less then $10 \%$ at the junction line is accepted, even if the penumbral shapes are unequal (junction between a collimator and MLC). In practice this means that an offset, or disagreement between light field and radiation field edge $(50 \%)$ at the central axis, of $0.5 \mathrm{~mm}$ or less will be accepted.

Patient measurements showed evident over- and underdosages across the junction, up to $133 \% \pm 3 \%$ (2SD). A $140 \%$ overdose at the junction equates to an overlap of approximately $2 \mathrm{~mm}$ at the junction line. The region of overdose around the match line 
is $3-4 \mathrm{~mm}$ wide ${ }^{13}$. The most likely cause of these over- and underdosages across the junction is patient movement during treatment. Patient movements can originate from muscle tension and breathing during positioning. Relaxing during the treatment affects the position of the patient in relation to the irradiation beam. Also variations in head position can affect the dose in the junction area.

Using MOSFET dosimeters appears to be a suitable method to measure the dose across a junction. The detectors are small; thus many detectors can be placed together in a relatively small area. However, the effect of overestimating the dose in the penumbra region has to be taken in account.

\subsection{CONCLUSIONS}

Based on the presented results, our recommendations for an adjacent field method, using a split beam technique with Multi Leaf Collimators are:

1. For individual fractions of a treatment significant deviations may be present at the junction. These deviations average out over repeated treatments to give relatively smooth dose distributions over the junction area;

2. One individual measurement in the junction area should not lead to any action, because random errors greatly affect the measurement;

3. The QA procedure to check the dose at the junction line should simulate the clinical situation as accurately as possible, in order to recognise deviations that occur in clinical situations. This means that the field size, collimator- and gantry angles used in the QA procedure must be similar to the clinical situation.

4. If using MOSFET detectors in a match line region, a correction has to be made to the measurement results, to correct for the overestimation of the detector in the penumbra region. 


\section{REFERENCES}

1. Abdel-Hakim K, Nishimura T, Takai M, Suzuki S, Sakahara H. Abutment region dosimetry for the monoisocentric three-beam split field technique in the head and neck region using asymmetrical collimators. Br j Radiol. 2002; 75(893):428-434.

2. Ahmad M, Nath R. Three-dimensional radiotherapy of head and neck and esophageal carcinomas: a monoisocentric treatment technique to achieve improved dose distributions. Int J Cancer 2001; 96(1): 55-65.

3. Alecu R, Loomis T, Aleca J, Ochran T. Guidelines on the implementation of diode in vivo dosimetry programs for photon and electron external beam therapy. Med Dosimetry 1999; 24 (1): 5-12.

4. Chuang CF, Verhey LJ, Xia P. Investigation of the use of MOSFET for clinical IMRT dosimetric verification. Med. Phys. 2002; 29 (6): 1109-1115.

5. Essers M, Battum L, Heijmen BJM. A linear diode array (JFD-5) for match line in vivo dosimetry in photon and electron beams; evaluation for a chest wall irradiation technique. Radiother Oncol. 2001; 61 (2): 185-192.

6. Fernandez EM, Shentall GS, Mayles WPM, Dearnaley DP. The acceptability of a multileaf collimator as a replacement for conventional blocks. Radiother Oncol. 1995; 36: 65-74.

7. Gladstone DJ, Chin LM. Automated Data Collection and Analysis System for MOSFET Radiation Detectors. Med Phys. 1991; 18: 542- 548.

8. Hughes RC, Huffman D, Snelling JV, Zipperian JE, Ricco AJ, Kelsey A. Miniature Radiation Dosimeter for In-Vivo Radiation Measurements. Int J Radiat Oncol Biol Phys. 1988; 14: 963-967.

9. Idzes $\mathrm{MH}$, Holmberg $\mathrm{O}$, Mijnheer BJ, Huizenga $\mathrm{H}$. Effect of set-up uncertainties on the dose distribution in the match region of supraclavicular and tangential breast fields. Radiother Oncol. 1998; 46 (1): 91-98

10. Meeks SL, Williams RO, Bova FJ, Mendenhall WM, Buatti JM. The midline dose distribution for a three-field radiotherapy technique. Med Dosimetry 1999; 24 (2): 91 98

11. Palta JR, Yeung DK, Frouhar V. Dosimetric considerations for a multileaf collimator system. Med Phys. 1996; 23 (7): 1219-1224.

12. Ramani R, Russell S, O'Brien P. Clinical dosimetry using MOSFET's. Int J Radiat Oncol Biol Phys. 1997; 37 (4); 959-964.

13. Rosenthal DI, MCDonough J, Kassaee A. The effect of independent collimator misalignement on the dosimetry of abutted half-beam blocked fields for the treatment of head and neck cancer. Radiother Oncol. 1998; 49(3):273-278.

14. Sanders M, Sayeg J, Coffey C, Patel P, Walsh J. Beam profile analysis using GafChromic films. Strereotact Funct Neurosurg 1993;61 Suppl 1:124-129.

15. Scalchi $P$, Francescon P. Calibration of a MOSFET detection system for 6 MV in vivo dosimetry. Int J Radiat Oncol Biol Phys. 1998; 40 (4): 987-993.

16. Soubra M, Cygler J. Evaluation of a dual metal oxide- silicon semiconductor field effect transistor detector as radiation dosimeter. Med Phys. 1994; 21(4): 567572. 
17. Zhu L, Kron T, Barnes K, Johansen S, O'Brien P. Junction of lateral and anterior fields in head and neck cancer: a dosimetric assesment of the monoisocentric technique (including reproducibility). Int J Radiat Oncol Biol Phys. 1998; 41(1): 227-232. 



\title{
C H A P T E R
}

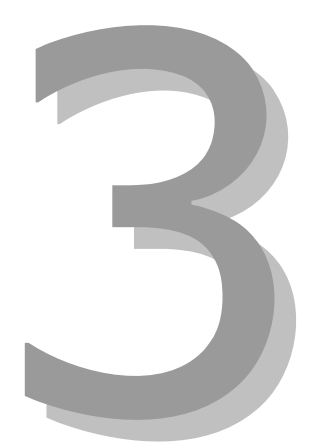

\section{Clinical implementation of MOSFET detectors for dosimetry in electron beams}

RADIOTHERAPY AND ONCOLOGY 80 (2006) 288-295

\author{
Esther J. Bloemen-van Gurp \\ Andre W. H. Minken \\ Ben J. Mijnheer \\ Cary J.G. Dehing- Oberye \\ Philippe Lambin
}




\section{ABSTRACT}

Background and purpose: To determine the factors converting the reading of a MOSFET detector placed on the patient's skin without additional build-up to the dose at the depth of dose maximum $\left(D_{\max }\right)$ and investigate their feasibility for in vivo dose measurements in electron beams.

Materials and methods: Factors were determined to relate the reading of a MOSFET detector to $D_{\max }$ for 4 to $15 \mathrm{MeV}$ electron beams in reference conditions. The influence of variation in field size, SSD, angle and field shape on the MOSFET reading, obtained without additional buid-up, was evaluated using 4,8 and $15 \mathrm{MeV}$ beams and compared to ionization chamber data at the depth of dose maximum $\left(z_{\max }\right)$. Patient entrance in vivo measurements included 40 patients, mostly treated for breast tumours. The MOSFET reading, converted to $D_{\max }$, was compared to the dose prescribed at this depth.

Results: The factors to convert MOSFET reading to $D_{\max }$ vary between 1.33 and 1.20 for the $4 \mathrm{MeV}$ and $15 \mathrm{MeV}$ beam, respectively. The SSD correction factor is approximately $8 \%$ for a change in SSD from $95 \mathrm{~cm}$ to $100 \mathrm{~cm}$, and $2 \%$ for each $5-\mathrm{cm}$ increment above $100 \mathrm{~cm}$ SSD. A correction for fields having sides smaller than $6 \mathrm{~cm}$ and for irregular field shape is also recommended. For fields up to $20 \mathrm{~cm} \times 20 \mathrm{~cm}$ and for oblique incidence up to $45^{\circ}$, a correction is not necessary. Patient measurements demonstrated deviations from the prescribed dose with a mean difference of $-0.7 \%$ and a standard deviation of $2.9 \%$.

Conclusion: Performing dose measurements with MOSFET detectors placed on the patient's skin without additional build-up is a well suited technique for routine dose verification in electron beams, when applying the appropriate conversion and correction factors. 


\subsection{INTRODUCTION}

Patient dose verification has been recommended for quality improvement of patient care in radiation therapy by several organisations such as the American Association of Physics in Medicine ${ }^{11}$, and the European Society of Therapeutic Radiology and Oncology, 23 . In vivo dosimetry has become an important part of a quality assurance (QA) programme in a radiotherapy department and has proven to be very useful in assessing the dose delivered to an individual patient, as well as in the detection of various types of errors in the dose delivery process ${ }^{7,13}$. Several dosimetry systems are available for use in radiotherapy. The most commonly used detector types for patient dose verification are diodes ${ }^{7,12,17,23}$ and thermo-luminescence dosimeters (TLDs) $)^{7,15,23}$. Recently there has been an increased interest in the use of the metal oxide-silicon semiconductor field effect transistor (MOSFET) for in vivo dosimetry purposes $1,2,3,4,5,6$, $9,14,18,19,20,21,22$. The system uses miniature non-intrusive MOSFET semiconductor radiation dosimeters (size less than $4 \mathrm{~mm}^{2}$ ). Other characteristics of MOSFET devices are the direct and simple dose readout, the portability of the system and the recording of the accumulated dose of each detector. The output can be converted from $\mathrm{mV}$ to cGy by entering a calibration factor in the reader for each single dosimeter. MOSFETs exhibit in addition a good linearity of their response $\mathrm{s}^{5,22}$.

Only limited information is available about the use of MOSFET detectors for dosimetric verification of radiotherapeutic treatments. After the initial evaluation of Soubra et al. ${ }^{22}$ showing that the characteristics of MOSFET detectors make them very promising for clinical dosimetry, only a few publications described their actual use in the clinic. Ramani et al. ${ }^{18}$ determined important physical characteristics of the detectors to demonstrate the usefulness of MOSFETs as clinical dosimeters. Measurements were performed at different sites of the patients' body, with both photon and electron beams of various energies. MOSFET and TLD measured dose values were compared and related to the expected dose. However, not many details (number of patients, the use of build-up, the presence of an insert or air gap, and the angular dependence during the electron beam measurements) are provided. Scalchi and Francescon ${ }^{21}$ calibrated MOSFET detectors to perform in vivo dosimetry during 6-MV treatments, both in normal set-up and during total body irradiation conditions. However, these authors did not present results of clinical studies. More recently the use of MOSFETs for clinical IMRT dosimetric verification has been reported ${ }^{5}$, but that study also concerned only phantom measurements.

Since January 2001, our department uses MOSFET dosimeters as part of a quality assurance programme of treatment delivery ${ }^{1}$. Since July 2002, in vivo dosimetry has been performed to check the entrance dose for all curative and elective treatments applying photon beams. In December 2002, we also implemented MOSFET dosimetry for $Q A$ of electron beams. 
In this paper we present the results of a systematic study of MOSFETs for measurements in electron beams. The goal of the study was to perform entrance in vivo measurements without additional build-up on the detector to prevent the target volume from shadowing by the build-up material of the detector. However, in most cases we are not interested in the dose at the surface. For this reason we converted the readings obtained with MOSFET dosimeters, placed on the patient's skin/ phantom without build-up, to absolute dose values of $Z_{\max }$ under reference conditions. A comprehensive set of phantom measurements was performed to determine these conversion factors. Measurements in the surface region are, however, sensitive to differences in energy and spatial distribution of electrons in clinical conditions compared to reference conditions. Supplementary measurements were performed to investigate the influence of these non-reference conditions, such as SSD, gantry angle, field size and shape on a MOSFET measurement, yielding an additional set of correction factors. Patient measurements were analysed with the aim to evaluate the use of MOSFET detectors under clinical conditions.

\subsection{MATERIALS AND METHODS}

\subsubsection{MOSFET dosimeters}

All measurements were performed using TN-502RD MOSFET dosimeters, produced by Thomson and Nielsen Electronics Ltd (Ottawa, Canada). For phantom measurements the MOSFET AutoSense system (TN-RD-60), developed for on-line radiotherapy applications, was used. The standard TN-RD-50 patient verification system, which does not allow on-line dose reading, was used for patient measurements. The two systems are virtually identical; the only difference is that the AutoSense system can be used for repetitive measurements as the control box of the MOSFET system can be read out without entering the treatment room.

The sensitivity of the MOSFET detector depends on the oxide thickness and on the gate voltage provided by the bias supply. Thomson and Nielsen have two types of MOSFET detectors, standard/ high sensitivity and two voltages on the bias supply, standard sensitivity $\left(1 \mathrm{mV} \mathrm{cGy}^{-1}\right)$ and high sensitivity $\left(3 \mathrm{mV} \mathrm{cGy}{ }^{-1}\right)$. At lower dose level ( $<1 \mathrm{~Gy}$ ) the high sensitivity bias and/ or the high sensitivity MOSFETs are recommended in order to cover this low dose range with an optimal reproducibility. However, the lower sensitivity dosimeters associated with the lower gate bias, enable the MOSFETs to be irradiated to a higher accumulated dose.

The TN502RD MOSFET dosimeter is a dual-bias dual-MOSFET detector. This kind of detector consists of two identical MOSFETs, fabricated on the same silicon chip and operating at two different gate biases (1V and 15V). During irradiation each sensor will produce a threshold voltage shift, the gate voltage necessary to allow charge 
conduction through the MOSFET. The reader measures and records the threshold voltages by passing current through the cable to the MOSFET chips. The difference between the two threshold voltage shifts is a function of absorbed dose and will be displayed on the reader. The bias supply provides a constant bias voltage on the MOSFET during irradiation. Details of the process involved in the use of a MOSFET as a dosimeter can be found elsewhere ${ }^{5}, 18,21,22$. The silicon chip (1 mm square, active area $0.2 \mathrm{~mm} \times 0.2 \mathrm{~mm}$ ) is covered with an epoxy bulb on top of the MOSFET gate oxide. The water equivalent thickness of the MOSFET is about $0.8 \mathrm{~mm}$ (flat side) and $1.8 \mathrm{~mm}$ (epoxy bulb side), under normal beam incidence in a $10 \times 10 \mathrm{~cm}^{2}$ field of a 6 MV X-ray beam at $100 \mathrm{~cm} \mathrm{SSD}{ }^{21}$.

For this study, a set of standard MOSFETs with the bias supply set at standard sensitivity was used. The MOSFETs were taped on the surface of the phantom, or skin of the patient, with the flat side of the detector facing the beam. After each irradiation the dose was read out by connecting the bias supply to the readout device. Before use, the MOSFETs were calibrated in a $6 \mathrm{MV}$ photon beam, as will be described below.

\subsubsection{Calibration procedure}

Calibration is performed to convert the dose-induced dosimeter voltage shift to cGy. It has to be executed prior to the first measurement with a new dosimeter and after any lengthy period of not being used. After applying the appropriate calibration factor $\left(F_{\text {cal }}\right)$, the system may yield dose values at specific sites ${ }^{18}$, including organs at risk and in junction areas ${ }^{1}$. Although the goal of the study was to perform entrance in vivo measurements in electron beams without build-up material on the detector, $F_{c a l}$ is obtained in photon beams under full build-up, to obtain maximum accuracy and repeatability. In our hospital the MOSFETs are first calibrated at depth in a photon beam, and the same detectors are then used for entrance in vivo dose measurements in both photon beams (with build-up) and electron beams (without build-up).

MOSFET calibration was performed using a $6 \mathrm{MV}$ photon beam, $10 \mathrm{~cm} \times 10 \mathrm{~cm}$ field size and $100 \mathrm{~cm}$ SSD. Five detectors were placed in a circle in a PMMA home-made calibration phantom at depth of $D_{\max }$, at minimal distance from the central axis of the beam. Ten measurements were performed and the results were averaged. We compensated for actual accelerator output by measuring this output with a calibrated cylindrical ionisation chamber (PTW type 31002, Physikalisch-Technische Werkstaetten, Freiburg, Germany), following the Dutch code of practice for the dosimetry of high-energy photon beams. $F_{\text {cal }}$ was determined as the ratio of the measured output voltage of the dosimeter and the actual $D_{\text {max }} . F_{\text {cal }}$ was entered into the MOSFET reader to obtain a reading in CGy. For entrance in vivo measurements in electron beams without build-up, conversion factors were established. 


\subsubsection{Phantom measurements}

Phantom measurements were performed (1) to determine the conversion factor $\left(F_{\text {conversion }}\right)$ between the readings of the MOSFET performed without additional build-up (based on photon calibration), to the $D_{\max }$ in electron beams (2.3.1.) and (2) to determine correction factors (CFs) for varying SSD, beam angle, field size and shape on the measurement result (2.3.2.). For all measurements, an Elekta SL-15i linear accelerator was used. Square applicators with dimensions ranging from $6 \mathrm{~cm}$ to $20 \mathrm{~cm}$ and a length of $42 \mathrm{~cm}$ were combined with inserts yielding field sizes ranging from 4 $\mathrm{cm}$ tot $20 \mathrm{~cm}$ square. The applicator is fitted to the surface of the phantom/ patient at $95 \mathrm{~cm} \mathrm{SSD}$. A radiation dose of $100 \mathrm{cGy}$ was delivered for $Z_{\max }$. The detector was placed with the round epoxy side of the detector on the surface of a PMMA phantom. The measurement results were compared to absolute dose values obtained with a Markus ionization chamber placed at the depth of $D_{\max }$ (PTW type M23343, Physikalisch-Technische Werkstaetten, Freiburg, Germany).

\subsubsection{Conversion factors}

For an accurate comparison of MOSFET reading with the prescribed dose (i.e. corresponding to $D_{\max }$ ), the MOSFET readings at the surface (performed without buildup) were converted to $D_{\max }$. Because the magnitude of $F_{\text {conversion }}$ depends on the energy of the beam, measurements were performed using 4, 6, 8, 10, 12 and $15 \mathrm{MeV}$ beams. Three MOSFET detectors were taped on a phantom and for each energy 10 measurements were performed under reference conditions $(100 \mathrm{~cm} \mathrm{SSD}$ and a $10 \mathrm{~cm}$ $x 10 \mathrm{~cm}$ applicator and insert size). Because in the clinical situation, the basic procedure is to irradiate patients at $95 \mathrm{~cm} \mathrm{SSD}$, we converted these factors to values at $95 \mathrm{~cm}$, using the correction for SSD (including the inverse square law). Markus chamber measurements were performed at $Z_{\max }$. The measurement results were averaged and $F_{\text {conversion }}$ was determined as:

Fconversion $(E)=\frac{\text { dose }\left(E, Z_{\text {max }}, 10 \times 10,95\right)}{R(E, \text { surface, } 10 \times 10,95) * F_{\text {cal }}(6 \mathrm{MV})}$

where $R$ is the MOSFET reading in $\mathrm{mV}$, with the MOSFET at the surface of the phantom. The dose at the depth of dose maximum for non-reference conditions, which is generally called entrance dose during in vivo dosimetry 8 , is now calculated from the reading of the MOSFET $(\mathrm{mV})$ at the surface, according to:

Dentrance $=R^{*} F_{\text {cal }}(6 \mathrm{MV}){ }^{*} F_{\text {conversion }}(\mathrm{E}){ }^{*} \Pi \mathrm{CFi}_{\mathrm{i}}(\mathrm{E})$

\subsubsection{Influence of varying SSD, beam angle, field size and shape}

Additional measurements were performed to determine $\mathrm{CFi}(\mathrm{E})$, the variation in the ratio between the reading of the MOSFET at the surface and the dose determined with an 
ionisation chamber placed at $z_{\max }$, when changing the reference conditions (SSD, angle of incidence, field size and field shape).

To investigate the influence of variation in SSD on the MOSFET reading, measurements were performed using an SSD varying from 95 to $110 \mathrm{~cm}$ for a $10 \mathrm{~cm}$ x $10 \mathrm{~cm}$ insert. Distances of 95, 100, 105 and $110 \mathrm{~cm} \mathrm{SSD}$ were used in an $8 \mathrm{MeV}$ electron beam, while measurements at 95 and $110 \mathrm{~cm} \mathrm{SSD} \mathrm{were} \mathrm{performed} \mathrm{for} 4$ and $15 \mathrm{MeV}$ electron beams. Three MOSFETs were taped at the centre of the field, on the surface of the phantom. Ten measurements were performed, and the results were averaged and related to the Markus chamber measurements performed at $Z_{\max }$.

Older studies reported a large angular dependence for MOSFETs in photon beams ${ }^{18,}$ 21 , although a more recent study showed a much smaller angular dependence, within $2.5 \%$ for gantry angles varying between 0 and 180 degrees $^{5}$. No information is available about the angular dependence of MOSFET detectors in electron beams. The angular dependence of the MOSFET detectors was therefore investigated by placing three MOSFETs at the centre of a PMMA cylindrical mini-phantom with $1.5 \mathrm{~cm}$ radius (Fig.1a). The mini-phantom was placed on a small Styrofoam block, which is almost air equivalent material, to approach free air geometry. The centre of the phantom was positioned in the middle of a $10 \mathrm{~cm} \times 10 \mathrm{~cm}$ electron field at $100 \mathrm{~cm}$ SDD, with the flat side of the detector facing the beam at $0^{\circ}$ gantry angle. Gantry angles were varied between 0 and $90^{\circ}$ in steps of $15^{\circ}$ for measurements in the $8 \mathrm{MeV}$ beam, and from $0^{\circ}$, $45^{\circ}$ to $90^{\circ}$ degrees using the 4 and $15 \mathrm{MeV}$ beams. Measurements were repeated 10 times for each gantry angle and the readings were related to the readings at $0^{\circ}$. The output variation of the linear accelerator as a function of gantry angle was determined to be less than $1 \%$ for the three beam energies.

Beside the earlier described non-isotropic response of the MOSFET, in the clinical situation the effect of gantry angle rotation on the MOSFET reading (performed without build-up) is due to two factors: the ratio MOSFET reading at the surface/ ionisation chamber at $z_{\max }$ and differences in the contribution of electron scatter from the surface of the phantom when changing the angle of incidence of the beam. To approach this situation and to investigate the influence of this scatter component, measurements were performed with the detector fixed on the flat surface of a rectangular PMMA phantom (Fig 1b.). Three MOSFET dosimeters were positioned at the centre of a 10 $\mathrm{cm} \times 10 \mathrm{~cm}$ field at $100 \mathrm{~cm}$ SSD. Gantry angles were varied between 0 and $90^{\circ}$ in steps of $15^{\circ}$ for measurements in the $8 \mathrm{MeV}$ beam, and from $0^{\circ}, 45^{\circ}$ to $90^{\circ}$ degrees using the 4 and $15 \mathrm{MeV}$ beams. Measurements were repeated 10 times for each gantry angle and the readings were related to the readings at $0^{\circ}$. 


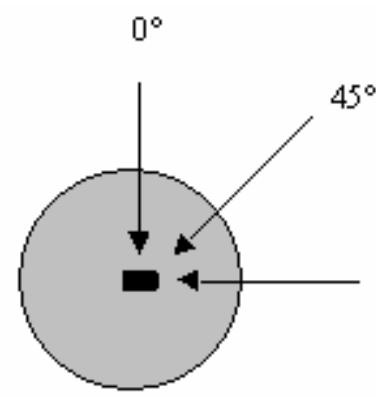

(a)

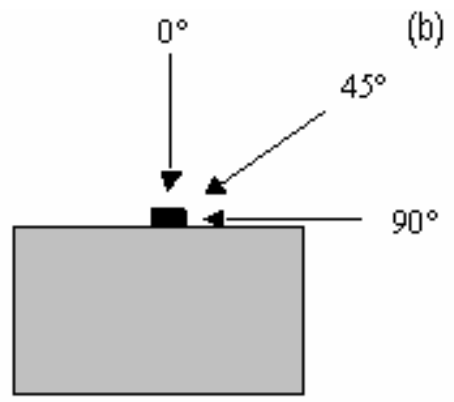

Figure 1: Experimental set-up for the determination of the angular dependence of the MOSFETs, using a cylindrical PMMA (a) and a flat phantom (b).

The influence of field size was investigated using $4 \mathrm{~cm} \times 4 \mathrm{~cm}$ and $6 \mathrm{~cm} \times 6 \mathrm{~cm}$ inserts ( $6 \mathrm{~cm} \times 6 \mathrm{~cm}$ applicator), $10 \mathrm{~cm} \times 10 \mathrm{~cm}$ insert $(10 \mathrm{~cm} \times 10 \mathrm{~cm}$ applicator) and $20 \mathrm{~cm} \times$ $20 \mathrm{~cm}$ insert $(20 \mathrm{~cm} \times 20 \mathrm{~cm}$ applicator) and the detectors were positioned at the surface of the phantom placed at $95 \mathrm{~cm} \mathrm{SSD} \mathrm{in} \mathrm{a} 8 \mathrm{MeV}$ electron beam. For the $4 \mathrm{~cm} \mathrm{x}$ $4 \mathrm{~cm}$ and $20 \mathrm{~cm} \times 20 \mathrm{~cm}$ field sizes with corresponding applicators also the $4 \mathrm{MeV}$ and $15 \mathrm{MeV}$ electron beams were investigated. Three MOSFET dosimeters were irradiated with a dose of $100 \mathrm{cGy}$ and for each situation 10 measurements were performed. lonization chamber measurements were performed at $Z_{\max }$, determined for each field size and energy. The MOSFET measurements were averaged and related to the ionization chamber results.

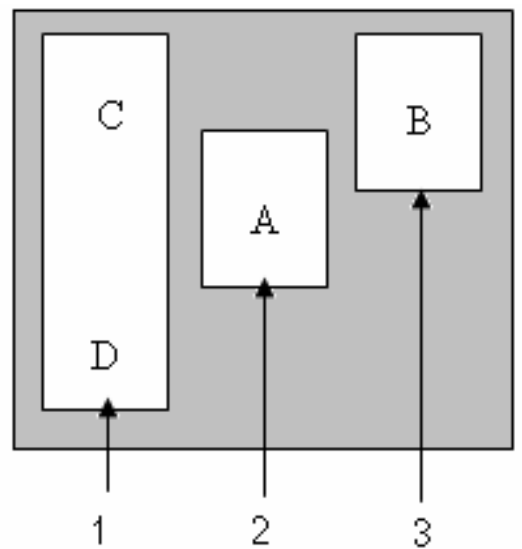

Figure 2: A shaped insert, made of Cerrobend, used to investifgate the influence of shape, size and position of the opening in the insert on the MOSFET reading. Numbers 1,2 and 3 are the cut-outs and $A, B, C$ and $D$ are the measuring locations. 
To investigate the influence of field position and field shape on the MOSFET response, a limited set of measurements was performed using a home-made insert for the $20 \mathrm{~cm}$ $x 20 \mathrm{~cm}$ applicator with three openings (Fig. 2). Measurements at the centre of opening 1 (location A) and 2 (location B) (both $6 \mathrm{~cm} \times 6 \mathrm{~cm}$ ) were performed to investigate the influence of field position in the applicator. Measurements close to the upper (location C) and lower edge (location D) of opening $3(5 \mathrm{~cm} \times 18 \mathrm{~cm}$ ) were performed to investigate the influence of field shape. Three MOSFET dosimeters were placed at the surface of the phantom for each location, using beam energies of 4, 8 and $15 \mathrm{MeV}$. Measurements were repeated 10 times, the results were averaged and related to ionization chamber measurements performed at $z_{\max }$ at the same distance from the field axis. The value of $z_{\max }$ was determined for a $6 \mathrm{~cm} \times 6 \mathrm{~cm}$ field size and 4,8 and 15 $\mathrm{MeV}$ electron beams. Based on data available in the literature, it was assumed that $Z_{\max }$ for a $6 \mathrm{~cm} \times 6 \mathrm{~cm}$ field size is almost identical to that of a $5 \mathrm{~cm} \times 18 \mathrm{~cm}$ field size ${ }^{16}$.

\subsubsection{Patient measurements}

Forty patients were included in the clinical study. The patients were irradiated at several locations; 31 on the breast/ thorax wall, 6 on the internal mammary lymph node region, 2 on the skull and one at the testis. The radiation dose per fraction ranged between 1.8 to 2.0 Gy and 6, 8, 10,12 and $15 \mathrm{MeV}$ electron beams were used. Field sizes varied between $6 \mathrm{~cm}$ and $20 \mathrm{~cm}$ diameter (round or square). For 9 patient treatments a customised insert was used, while for 14 patients an air gap between the insert and the skin, ranging between 0.5 and $4 \mathrm{~cm}$, was present (SSD 95.5 up to 99 $\mathrm{cm})$.

Patient entrance in vivo measurements were performed during the first fraction of the treatment. The detectors were located at the centre of the insert, with the round epoxy side of the detector in contact with the skin, for every beam given to the patient. The SSD at the centre of the field was measured for each beam. After the application of the MOSFET and the measurement of the SSD, the patient was irradiated and after the completion of the beam the MOSFET was read out. The MOSFET reading at the

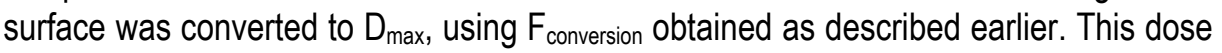
obtained from the MOSFET measurement, recalculated to $Z_{\max }$ and corrected for nonreference conditions $\left(D_{\text {entrance}}\right)$, was compared to the prescribed dose $\left(D_{\text {plan }}\right)$. The deviation is presented as $\left(D_{\text {entrance }}-D_{\text {plan }}\right) / D_{\text {plan }}{ }^{*} 100$. In 8 patients the electron beam was given in combination with one or more photon beams within a fraction and the number of monitor units (MUs) was calculated using the FOCUS treatment planning system version 3.0 (CMS Inc., Saint Louis, MO, USA). FOCUS applies a modified version of the Hogstrom dose calculation algorithm. For the other patient irradiations, where the electron beam was given alone, a home-made interpolation program based on measurements was used to calculate the radiation dose. A maximum deviation of about $3 \%$, based on experimental verification, can be attributed to these calculations due to limitations of this algorithm. 


\subsection{RESULTS}

\subsubsection{Phantom measurements}

$F_{\text {cal }}$ for the individual dosimeters ranged from 1.05 to 1.10 . The mean reproducibility of $F_{\text {cal }}$ and $C F s$ is about $2.0 \%$ (1 SD) in photon beams with full equilibrium, 10 successive measurements at about $100 \mathrm{mV}$ and standard sensitivity.

\subsubsection{Conversion factors ( $\left.F_{\text {conversion }}\right)$}

The results of the measurements shown in Fig. 3 demonstrate that the ratio of the MOSFET reading at the surface (in cGy) and the ionisation chamber dose at $z_{\max }$ increases with increasing beam energy. The result varies between $0.75 \pm 0.02$ and $0.83 \pm 0.02(1 \mathrm{SD})$ for an energy range of $4-15 \mathrm{MeV}$. This means that $F_{\text {conversion, }}$ necessary to convert the MOSFET reading to $D_{\max }$, decreases with increasing energy of the beam. $F_{\text {conversion is }} 1.33$ for the 4 and $6 \mathrm{MeV}$ beam and 1.30, 1.26, 1.24 and 1.20 for the $8,10,12$ and $15 \mathrm{MeV}$ beams, respectively, at $95 \mathrm{~cm} \mathrm{SSD}$ for a $10 \mathrm{~cm} \times 10 \mathrm{~cm}$ field size. The reproducibility of the MOSFET detector measurements varied between $1.1 \%$ and $2.5 \%$ (1 SD).

\subsubsection{Influence of varying SSD, beam angle, field size and shape $\left(\mathrm{CF}_{\mathrm{i}}\right)$}

The ratio of the MOSFET reading at the surface/ ionisation chamber dose $\left(z_{\max }\right)$, decreases with increasing SSD, which would result in an underestimate of the entrance dose by the MOSFET. The results are shown in Fig. 4a. The decrease of the ratio MOSFET reading at the surface /ionisation chamber dose $\left(z_{\max }\right)$ is $8.3 \%$ for the $8 \mathrm{MeV}$ beam, for an SSD variation from $95 \mathrm{~cm}$ to $100 \mathrm{~cm} \mathrm{SSD} \mathrm{(ratio} 0.92 \pm 0.03$ ). Further increase to $110 \mathrm{~cm} \mathrm{SSD} \mathrm{gives} \mathrm{a} \mathrm{smooth} \mathrm{underestimation} \mathrm{of} \mathrm{(1.7 \% ),} \mathrm{up} \mathrm{to} \mathrm{a} \mathrm{ratio} \mathrm{of}$ $0.90 \pm 0.03$ (1 SD). The $4 \mathrm{MeV}$ beam gives a $10.4 \%$ underestimation in dose for an SSD variation from $95 \mathrm{~cm}$ to $110 \mathrm{~cm} \mathrm{SSD} \mathrm{(ratio} 0.90 \pm 0.03$ ). For the $15 \mathrm{MeV}$ beam an underestimation of $7.4 \%$ is observed $(0.93 \pm 0.03)$. These measurements prove that a correction for SSD is necessary, especially for SSD up to $100 \mathrm{~cm}$.

The results of the measurements performed using a cylindrical mini-phantom, demonstrate that the angular dependence of the MOSFET detector in electron beams is small. For the $4 \mathrm{MeV}$ beam the reading decreases $3.2 \%$ with increasing gantry angle: from $61.6 \pm 1.3 \mathrm{cGy}$ at $0^{\circ}$ to $60.3 \pm 1.4 \mathrm{cGy}$ at $45^{\circ}$, and $59.6 \pm 1.1 \mathrm{cGy}$ at $90^{\circ}$. For the $8 \mathrm{MeV}$ beam, the variation in detector response with gantry angle is negligible (within $1.0 \%$ ). Also for the $15 \mathrm{MeV}$ beam, the angular dependence of the detector is small, within $2.9 \%$ : from $88.5 \pm 1.4 \mathrm{cGy}$ at $0^{\circ}$ to $89.7 \pm 1.7 \mathrm{cGy}$ at $45^{\circ}$ and $87.1 \pm 2.0$ cGy at $90^{\circ}$. 


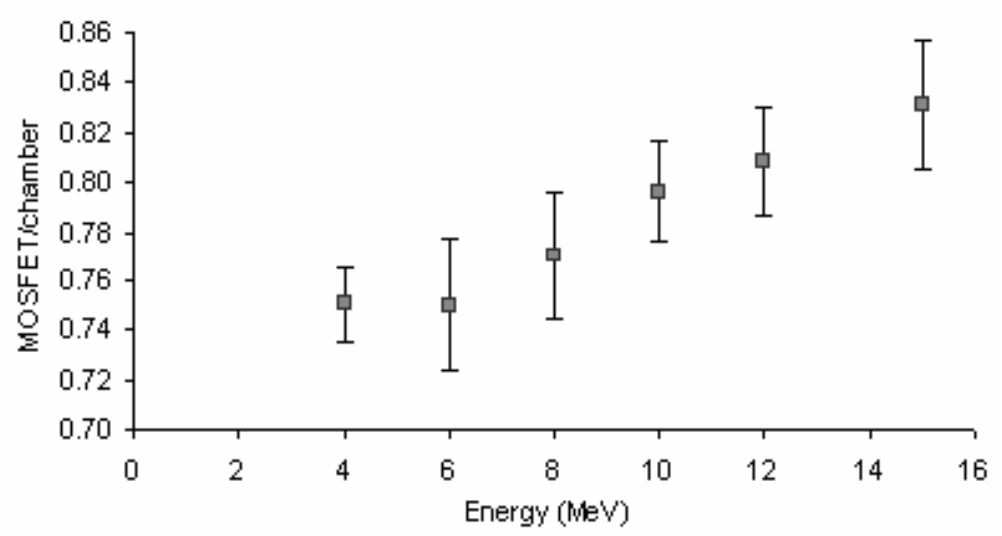

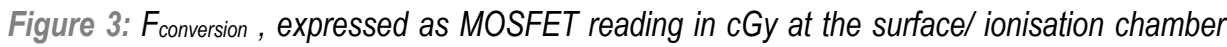
dose at the depth of dose maximum $\left(D_{\max }\right)$ in $c G y$, for 4, 6, 8, 10, 12 and $15 \mathrm{MeV}$ beams at 95 $\mathrm{cm} S S D \mathrm{~cm}$ and a $10 \mathrm{~cm} \times 10 \mathrm{~cm}$ applicator. The mean result of 10 measurements using three MOSFETs and the standard deviation are given.

The results of the measurements investigating the MOSFET reading at the surface lionisation chamber dose $\left(D_{\max }\right)$ and the contribution of electron scatter during gantry rotation are shown in Fig. 4b. The variation in dose caused by a variation in angle towards $45^{\circ}$, the range of angles that is clinically most important for electron beams, is within $3 \%$ for 4,8 and $15 \mathrm{MeV}$ beams. The ratio of the readings at 45-degree gantry angle and at 0 degrees are for both MOSFET 1 and 3, $1.03 \pm 0.03,0.99 \pm 0.02$ and $1.00 \pm 0.03$ ( $1 \mathrm{SD}$ ), for the 4,8 and $15 \mathrm{MeV}$ beams, respectively. The ratio of the readings for MOSFET 2 is $1.14 \pm 0.02,1.06 \pm 0.02,1.04 \pm 0.01$ ( 1 SD), for the same energies. For larger angles towards $75^{\circ}$, an increase of reading was detected for all MOSFETs. The average ratio of the reading at $75^{\circ}$ compared to that at zero degrees is for the three MOSFETs at $8 \mathrm{MeV} 1.24 \pm 0.03$ (1 SD). The results of MOSFET 2 were kept out of this analysis, because of its abnormal behaviour compared to the other detectors. For gantry angles up to $45^{\circ}$, a correction is not necessary. For gantry angles larger than $45^{\circ}$, a significant deviation was demonstrated; however these angles are not very relevant in clinical situations.

The ratios of the MOSFET reading at the surface/ ionisation chamber dose (Dmax) for changes in field size are shown in Fig. 4c. The value of $F_{\text {conversion }}$ does not depend on field size, for field sizes larger than $6 \mathrm{~cm} \times 6 \mathrm{~cm}$. For fields smaller than $6 \mathrm{~cm} \times 6 \mathrm{~cm}$, the MOSFET overestimates the entrance dose by about $5 \%$ depending on the electron beam energy. Using $4 \mathrm{MeV}$ beams, the ratio is almost independent of field size, while for a field size of $4 \mathrm{~cm} \times 4 \mathrm{~cm}$ for the $8 \mathrm{MeV}$ and $15 \mathrm{MeV}$ beam the ratio is 1.05 and 1.06 , respectively. 

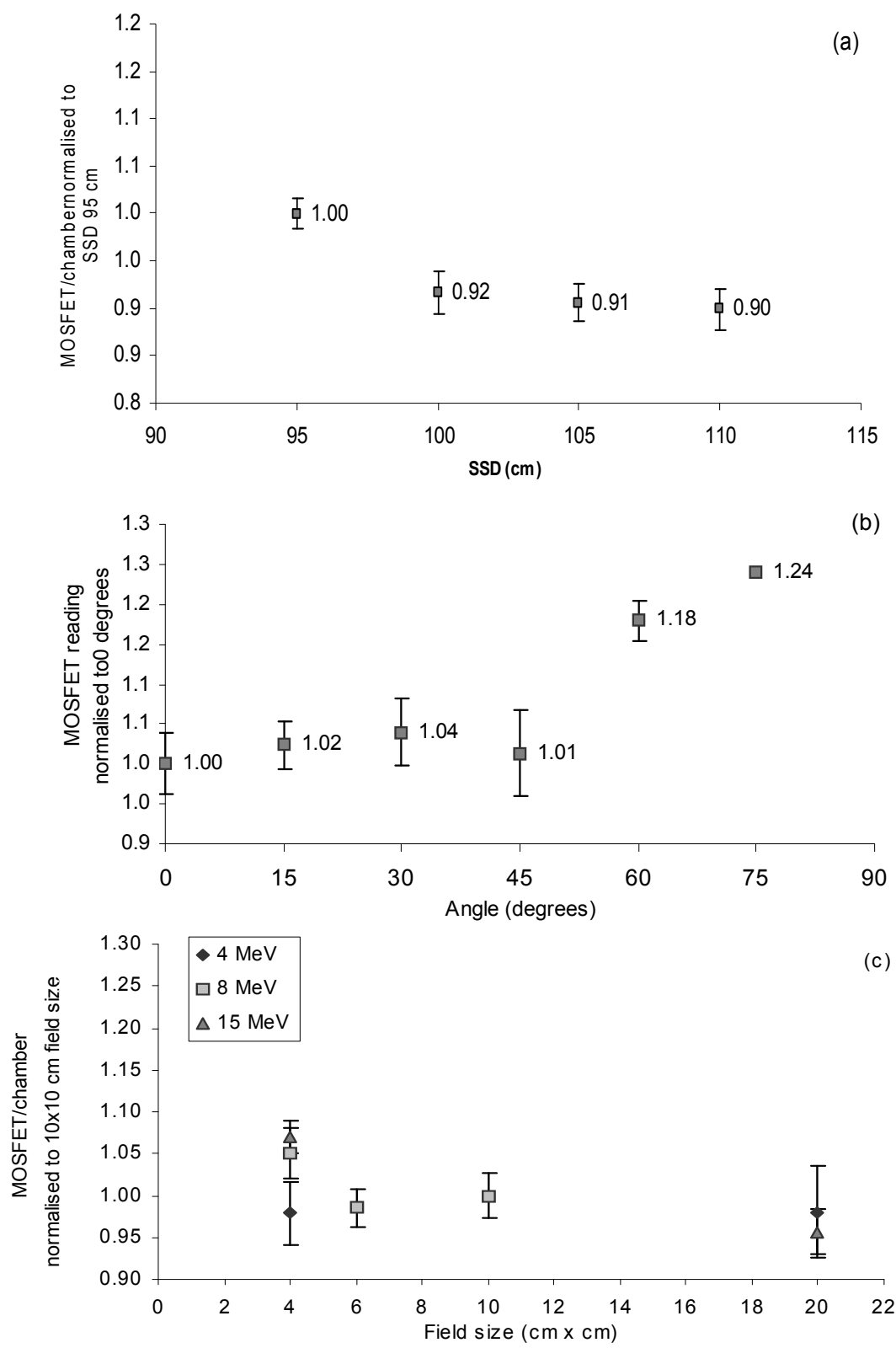

Figure 4a: MOSFET reading at the surface/ ionisation chamber dose ( $\mathrm{Zmax})$, as a function of $S S D$ and normalized to $95 \mathrm{~cm}$ SSD for $8 \mathrm{MeV}$ electron beams. b: Angular dependence of MOSFET reading at the surface, normalized to 0 degrees using $8 \mathrm{MeV}$ electron beams. c: MOSFET reading at the surface/ ionisation chamber dose ( $\left.\mathrm{Z}_{\max }\right)$ as a function of field size and normalized to a $10 \mathrm{~cm} \times 10 \mathrm{~cm}$ field size, for 4, 8 and $15 \mathrm{MeV}$ electron beams. The mean result of 10 measurements using three MOSFETs and the standard deviation are given in these three figures. 
The shape of the insert and the position of the dosimeter in the radiation field, affect a MOSFET measurement without additional build-up. The magnitude of this effect depends on the energy of the beam. The influence of field position and field shape on the MOSFET response is within $4.0 \%$ for the $4 \mathrm{MeV}$ beam. The ratios of the relative readings for locations $B, C$ and $D$, compared to location $A$ for the $4 \mathrm{MeV}$ beam are: $0.99 \pm 0.01,1.02 \pm 0.02$ and $1.04 \pm 0.02$ ( $1 \mathrm{SD}$ ), respectively. For the $8 \mathrm{MeV}$ beam the ratios of the readings are $1.01 \pm 0.01,0.95 \pm 0.03$ and $1.04 \pm 0.02(1 \mathrm{SD})$ and for the $15 \mathrm{MeV}$ beam $0.94 \pm 0.04,0.95 \pm 0.03$ and $0.95 \pm 0.03$ ( $1 \mathrm{SD}$ ). These results demonstrate that for the lower energies (4 and $8 \mathrm{MeV}$ ) there is no significant deviation in MOSFET reading during variation in field position $(B)$ and shape $(C$ and $D)$. For higher energies $(15 \mathrm{MeV})$ however, the reading decreases in these situations with 5.9 $\%$.

\subsubsection{Patient measurements}

MOSFET entrance in vivo measurements were performed during the first treatment session of 40 patients. The MOSFET reading at the surface was converted to dose at

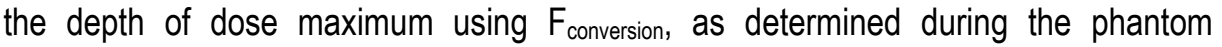
measurements. Field sizes varied between $6 \mathrm{~cm}$ and $20 \mathrm{~cm}$ diameter (round or square) and therefore a field size correction was not necessary. For 14 patients a correction for SSD was applied for distances between the insert and the skin ranging between 0.5 and $4 \mathrm{~cm}$. The patient measurements $\left(D_{\text {entrance }}\right)$, first converted to $D_{\max }$ and then corrected for SSD, were compared with the prescribed dose $\left(D_{\text {plan }}\right)$.

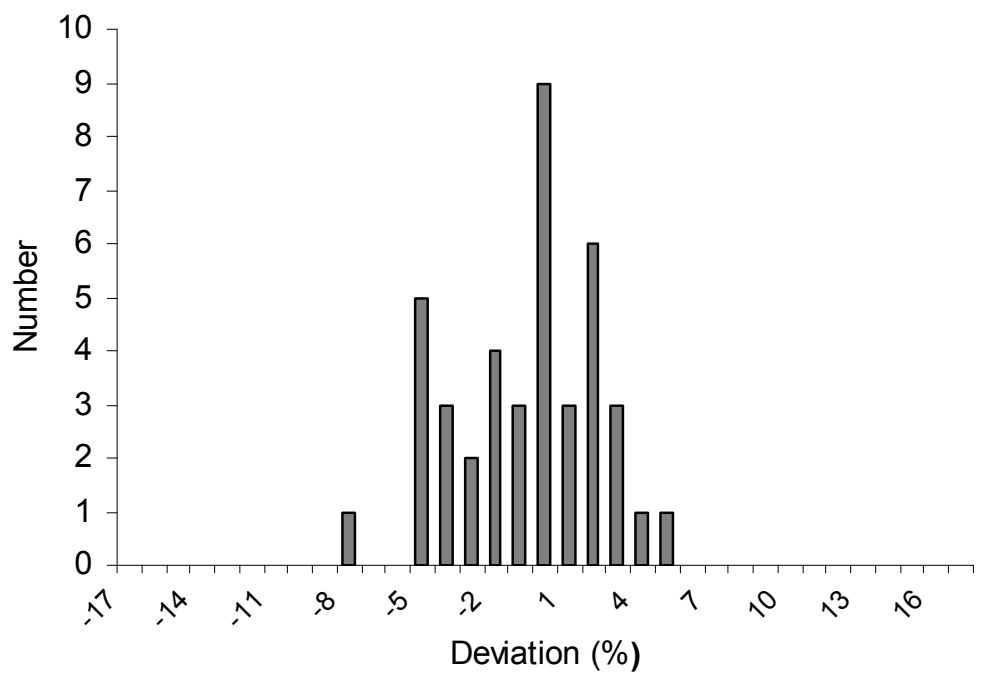

Figure 5: The distribution of dose deviations for 40 patients irradiated with electron beams, calculated as the MOSFET reading converted to $D_{\max }$ and corrected for SSD, compared with the prescribed $D_{\max }$. 
The deviations between the prescribed dose values and the MOSFET results ranged between $-8.1 \%$ and $4.7 \%$. The mean result of all measurements was $-0.7 \%$ with a standard deviation of $2.9 \%$. The data for all 40 patients are presented in Fig. 5.

\subsection{DISCUSSION AND CONCLUSION}

The ratio of the MOSFET reading at the surface and the ionisation chamber dose at the depth of $D_{\max }$ increases with increasing beam energy. This variation may be caused, in part, by the lower surface dose in lower energy electron beams. However part of it could also be caused by an energy dependence.

This study shows that the mean MOSFET reproducibility is $2.0 \%$ (1 SD) for readings of $100 \mathrm{mV}, 10$ successive measurements and standard sensitivity of the bias supply. This is of the same order of magnitude as observed by other groups. Jornet et al. determined an intrinsic precision of MOSFETs working in the standard sensitivity mode of $2.5 \%$ (1 SD) for doses of approximately $1 \mathrm{~Gy}^{9}$. At higher doses the precision of the detector increases. Ramani et al. performed measurements with a MOSFET at standard sensitivity and readings of $2 \mathrm{~Gy}$, measuring a standard deviation of the readings of $1 \%{ }^{18}$. The overall uncertainty in dose determination using the above described calibration methodology is about 4\% (1SD). This uncertainty is determined as the root-sum-square of the individual errors of $C F, F_{\text {conversion, }} F_{\text {cal }}$ and $R$, which are all estimated to be about $2 \%$, given as $1 \mathrm{SD}$.

By using the MOSFETs without any build-up, less shadowing by the detector of the target volume occurs. The price to be paid for this advantage is that the detector becomes more sensitive for electrons scattered by the collimating system and the insert. This is reflected in a rather strong dependence of the MOSFET response on SSD. As a consequence, for each patient measurement, the SSD has to be measured to perform a correction. This absence of build-up led also to the insert- shape dependence and higher readings for fields smaller than $6 \mathrm{~cm} \times 6 \mathrm{~cm}$, which is probably caused by the electrons scattered from the edge of the insert.

The angular dependence of the MOSFET response as measured inside a miniphantom is small. Up to 45 degrees the angular dependence can be ignored, whilst at larger angles the effect is about $3 \%$ at maximum and seems to be somewhat energy dependent. This small angular dependence was also observed by Chuang et al. ${ }^{5}$, and was explained by these authors as a result of improvement in detector design compared to previous detectors ${ }^{18,21}$. The increment in dose, measured with the MOSFET detector at the surface of the phantom without additional build-up, may be caused by an increase in the contribution of electrons scattered from the phantom into the detector, leading to a reading that increases with the angle of irradiation. 
The importance of the role of quality assurance in radiotherapy is generally accepted, due to the complexity and many steps involved in the radiotherapeutic process. However, the workload involved in developing and assessing an in vivo dosimetry programme, is often a problem for a radiotherapy department. In our institution we developed an in vivo dosimetry protocol, using MOSFETs, for all curative and elective photon and electron beam treatments, and for special indications such as the determination of the dose in organs at risk, or for junction measurements ${ }^{1}$. Due to the intensive use of MOSFETs for in vivo dosimetry purposes in our institution, special attention is therefore paid to making it an easy to use technique with acceptable accuracy.

The use of the detector is simple and in vivo dosimetry using MOSFETs is easy to integrate into the work processes. In our institution the radiation technologists perform the patient measurements. We developed a user interface in which the radiation technologists have to type in some specific treatment data. This program automatically converts the reading to $D_{\max }$ and corrects for the influence of SSD. The deviation of the measurement is given as a percentage of the prescribed dose. Action levels of $\pm 7-10$ $\%$, depending on the treatment site, are applied. These action levels are based on our experience with entrance dose measurements in photon beams and defined as the level were $85 \%$ of the measurements are within the action level during the first measurement. The result of this study demonstrates that the overall uncertainty in dose determination for entrance in vivo dosimetry in electron beams is about $4 \%, 1 \mathrm{SD}$. Action levels of $7 \%$ for all treatment sites, treated with electron beams using the described methodology, are therefore adequate. If deviations exceed the action level, all treatment data are checked, a physicist will evaluate the measurement result, and the measurement has to be repeated.

In this study we performed a comprehensive set of phantom measurements to determine CFs with a reproducibility of about $2 \%$ (1 SD), using standard bias voltage, standard MOSFETs and a radiation dose corresponding to $100 \mathrm{MUs}$. These CFs can be taken the same for all MOSFETs of a certain batch. However, some MOSFET detectors may show a deviating behaviour, in non-reference conditions. In our situation, one MOSFET responded differently to gantry angle rotation during the phantom measurements. For estimating generally applicable CFs, this is a practical difficulty. We decided not to take individual CFs into account, which may introduce some additional uncertainty. The inaccuracy that is introduced using such a procedure is accepted in order to keep the workload adequate and is taken into account in our tolerance levels. To evaluate the inter-batch variation and variation in MOSFET design we recommend to repeat part of the phantom measurements periodically.

When performing entrance in vivo measurements without build-up, $F_{\text {conversion is }}$ necessary to convert the MOSFET reading at the skin to $D_{\max }$, whilst a correction for SSD and small fields $(<6 \mathrm{~cm})$ also has to be applied. To estimate accurate conversion 
and CFs, an extensive set of phantom measurements has to be performed, for which an on-line system is recommended 10,19 .

The results of this study demonstrate that the characteristics of MOSFETs in electron beams (reproducibility, field size and angular dependence) make them well suited for in vivo dosimetry. Under these circumstances, in vivo dosimetry measurements can give supplementary information about the actual absorbed dose at a specified point. In addition, due to the current lack of electronic data transfer in our department, this method has become an essential part of our quality assurance programme.

As a result of this study, protocols for in vivo entrance dose measurements of electron treatments are developed and all patients treated with curative intent will undergo these measurements.

\section{ACKNOWLEDGEMENTS}

We are thankful to Thomson and Nielsen and PI-Medical, their Dutch representative, for providing us with the automated reader for this study. 


\section{REFERENCES}

1. Bloemen-van Gurp EJ, du Bois W, Visser PA, et al. Clinical dosimetry with MOSFET dosimeters to determine the dose along the field junction in a split beam technique. Radiother. Oncol. 2003; 67: 351-357.

2. Bower MW, Hintenlang DE. The characterization of a commercial MOSFET dosimeter system for use in diagnostic x ray. Health Phys. 1998; 75: 197-204.

3. Brauer-Krisch E, Bravin A, Lerch M, Rosenfeld A, Stepanek J, Di Michiel M, Laissue JA. MOSFET dosimetry for micro beam radiation therapy at the European Synchrotron Radiation Facility. Med. Phys. 2003; 30: 583-589.

4. Butson MJ, Rozenfeld A, Mathur JN, et al. A new radiotherapy surface dose detector: The MOSFET. Med. Phys. 1996; 23: 655-658.

5. Chuang CF, Verhey LJ, Xia P. Investigation of the use of MOSFET for clinical IMRT dosimetric verification. Med. Phys. 2002; 29: 1109-1115.

6. Cheung T, Butson MJ, Yu PK. Effects of temperature variation on MOSFET dosimetry. Phys. Med. Biol. 2004; 49: N191-196.

7. Essers M, Mijnheer BJ. In vivo dosimetry during external photon beam therapy: a critical review. Int. J. Radiat. Oncol. Biol. Phys. 1999; 43: 245-259.

8. Huyskens D, Bogaerts R, Verstraete J, et al. Practical Guidelines for the Implementation of in vivo Dosimetry with Diodes in External Radiotherapy with Photon Beams (Entrance Dose). Physics for Clinical Radiotherapy Booklet № 5 2001; ESTRO: Brussels: Belgium.

9. Jornet N, Carrasco P, Jurado D, Ruiz A, Eudaldo T, Ribas M. Comparison study of MOSFET detectors and diodes for entrance in vivo dosimetry in $18 \mathrm{MV}$ x-ray beams. Med. Phys. 2004; 31: 2534-2542

10. Kron T, Rosenfeld A, Lerch M, Bazley S. Measurements in radiotherapy beams using on-line MOSFET detectors. Radiat.Prot. Dosim. 2002; 101 N1/4:445-448.

11. Kutcher GJ, Coia L, Gillin M, et al. Comprehensive QA for Radiation Oncology: report of AAPM Radiation Therapy Committee Task Group 40. Med. Phys.1993; 21: 581618.

12. Lee PC, Sawicka JM, and Glasgow GP. Patient dosimetry quality assurance program with a commercial diode system. Int J. Radiat. Oncol. Biol. Phys. 1994; 29: 11751182.

13. Leunens G, Van Dam J, Dutreix A, et al. Quality assurance in radiotherapy by in vivo dosimetry. Determination of the target absorbed dose. Radiother. Oncol. 1990; 19: 73-87.

14. Marcié S, Charpiot E, Bensadoun R, Ciais G, Hérault J, Costa A, Gérard J. In vivo measurements with MOSFET detectors in oropharynx and nasopharynx intensitymodulated radiation therapy. Int J. Radiat. Oncol. Biol. Phys. 2005; 61: 1603-1606.

15. Mc Ghee P, Humphreys S, Dunscombe P. An efficient approach to routine TL dosimetry. Med. Dosim. 1993; 18: 187-191.

16. Niroomand-Rad A. Film dosimetry of small elongated electron beams for treatment planning. Med. Phys.1989; 16: 655-662.

17. Noel A, Aletti P, Bey $P$, et al. Detection of errors in individual patients in radiotherapy by systematic in vivo dosimetry. Radiother. Oncol. 1995; 34: 144-151. 
18. Ramani R, Russell S, O'Brien P. Clinical dosimetry using MOSFETs. Int. J. Radiat. Oncol. Biol. Phys. 1997; 37: 959-964.

19. Ramaseshan R, Kohli KS, Zhang TJ, Lam T, Norlinger B, Hallil A and Islam M. Performance characteristics of a microMOSFET as an in vivo dosimeter in radiation therapy. Phys. Med. Biol. 2004; 49: 4031-4048.

20. Rosenfeld AB, Lerch MLF, Kron T, Brauer-Krisch E, Bravin A, Holmes- Siedle A. Feasibility study of on- line, high spatial resolution MOSFET dosimetry in static and pulsed X-ray radiation fields. IEEE Trans. Nucl. Sci. 2001; NS-48 N6: 2061-2068.

21. Scalchi $P$, Francescon P. Calibration of a MOSFET detection system for $6 \mathrm{MV}$ in vivo dosimetry. Int. J. Radiat. Oncol. Biol. Phys. 1998; 40: 987-993.

22. Soubra M, Cygler J, Mackay G. Evaluation of a dual bias dual metal oxide silicon semiconductor field effect transistor detector as a radiation dosimeter. Med. Phys. 1994; 21: 567-572.

23. Van Dam J, Marinello G. Methods for in vivo dosimetry in external radiotherapy. Physics for Clinical Radiotherapy Booklet No 1 1994; ESTRO: Brussels: Belgium. 


\title{
CHAPTER
}

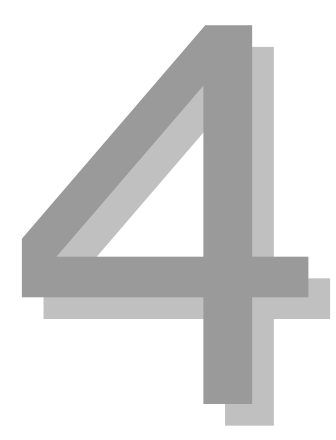

\section{Total body irradiation, towards optimal individual delivery: dose evaluation with MOSFETs, TLDs and a treatment planning system}

Int. J. Radiation Oncology Biol. Phys., Vol. 69, No. 4, pp $1297-$ 1304, 2007

\author{
Esther J. Bloemen-van Gurp \\ Andre W. H. Minken \\ Ben J. Mijnheer \\ Cary J.G. Dehing- Oberye \\ Philippe Lambin
}




\section{ABSTRACT}

Background and purpose: Predicting the 3-dimensional (3D)-dose distribution of our Total Body Irradiation (TBI) technique, using a commercial Treatment Planning System (TPS). In vivo dosimetry, using MOSFETs and thermoluminescence detectors (TLDs), was used to verify the calculated dose distributions.

Materials and methods: A total body CT scan was performed, loaded into our TPS and a 3D-dose distribution was generated. In vivo dosimetry was performed at five locations on the patient. Entrance and exit dose values were converted to midline doses using conversion factors, previously determined with phantom measurements. TPS predicated dose values were compared with MOSFET and TLD in vivo dose values.

Results: MOSFET and TLD dose values agreed within $3.0 \%$; MOSFET and TPS data within $0.5 \%$. The convolution algorithm of the TPS, which is routinely applied in the clinic, overestimated the dose in the lung region. Using a superposition algorithm reduced the calculated lung dose with approximately $3 \%$. The dose inhomogeneity as predicted by the TPS, can be reduced by using a simple IMRT technique.

Conclusions: The use of a TPS for calculating dose distributions in individual patients during TBI treatments is strongly recommended. Using a TPS gives good insight in over- and under-dosage in the patient and the influence of patient positioning on dose homogeneity. MOSFETs are suitable for in vivo dosimetry purposes during TBI, when using appropriate conversion factors. MOSFET, TLD and TPS results agree within acceptable margins. 


\subsection{INTRODUCTION}

Total Body Irradiation (TBI) is a complex radiotherapeutic technique, used for the treatment of certain haematological, oncologic, and immunologic diseases. The role of $\mathrm{TBI}$ is to destroy the recipient's bone marrow and tumour cells and to immunosuppress the patient sufficiently to avoid rejection of the donor bone marrow transplant. The dose in a TBI treatment is often prescribed to a point inside the body in the pelvic region at the height of the umbilicus, referred to as the dose prescription point ${ }^{1}$. It is generally assumed that the dose in the rest of the body should be within $10 \%$ of this prescribed dose ${ }^{1}$. Mostly, Monitor Unit (MU) calculation is done by point-dose determination at the dose prescription point. Computed Tomography (CT)-based treatment planning, however, can provide a much more detailed calculation of dose delivery to the whole body of the patient. This is important because of the special irradiation technique, the uncertainties in patient positioning and the fact that the quality of life and long term side effects become more and more important with the increasing survival rates ${ }^{2}, 3$. Most treatment planning systems do not allow the set up of patients at a very large SSD, because the system is not designed for these techniques and has to be validated under these circumstances. A set of phantom measurements has therefore to be performed, to check the accuracy under TBI conditions. Several authors described the use of a TPS for TBI treatments, based on home-made CT-aided PC-based planning systems ${ }^{4}$ and conventional CT-based treatment planning systems ${ }^{5-9}$. These groups demonstrated that a commercially available TPS is in principle suitable for predicting the dose distribution in a patient under TBI conditions. However, the use of a TPS for the calculation of a 3D-dose distribution in the patient's total body, correlated to individual patient treatment data and patient positioning procedures, has not been described. Abraham et al. ${ }^{9}$ evaluated the positioning of the arms related to the lung dose, however, the influence of position on other structures was not investigated.

It was the first aim of this study to investigate whether TBI treatments could be optimized by using 3D treatment planning of individual patients.

In vivo dosimetry during TBI gives the opportunity to check the dose in the patient and to verify whether predicted and measured dose values correspond within certain levels, but is difficult to perform due to the complexity of the irradiation technique. To obtain meaningful results, a TBI in vivo dosimetry method must first be validated with phantom experiments before it can be implemented clinically. TLDs have been proven to be useful detectors for measurements under TBI circumstances due to their small dependence on direction, dose rate, temperature, and energy in the therapeutic range, and their wide applicable dose range ${ }^{6,10-14}$. Accuracies within 3-4 \% (1SD) can be reached ${ }^{10-14}$. Compared to TLDs, the Metal Oxide Field Effect Transistor (MOSFET), a relatively new kind of detector, has the advantage of direct dose readout. Earlier publications described important physical characteristics of these detectors to demonstrate the usefulness of MOSFETs as a clinical dosimeter of radiotherapeutic 
treatments with photon ${ }^{15-24}$, electron ${ }^{25}$ and proton $^{26}$ beams, junction of fields ${ }^{27}$, IMRT fields ${ }^{19}, 28,29$ brachytherapy ${ }^{30-32}$ and intra-operative irradiation ${ }^{33}$, 34. The system uses miniature non-intrusive MOSFET semiconductor radiation dosimeters (size less than $4 \mathrm{~mm}^{2}$ ) and shows a good linearity of the response with no sensitivity variation with temperature and accumulated dose in the therapeutic high-energy range ${ }^{15}, 28,35,36$. Reports about the clinical use of MOSFET dosimeters for in vivo measurements during TBI treatments are, to the best of our knowledge, not available. Scalchi and Francescon ${ }^{37}$ calibrated MOSFET detectors to perform in vivo dosimetry during 6-MV treatments, both in normal set-up and during TBI conditions. However, these authors did not present results of clinical studies.

The second aim of this paper was to use both MOSFETs and TLDs for the in vivo verification of the actual delivered dose and to compare these values with the 3D-dose distribution calculated for our TBI technique by a commercial TPS.

\subsection{MATERIALS AND METHODS}

\subsubsection{Treatment planning system}

For this study, our clinically applied TPS was used (XiO 4.3.0, CMS Inc., Saint Louis, MO, USA), to evaluate the dose distribution in the full patient's body. In our institution we apply routinely the so-called Fast-Fourier Transform Convolution (FFTC) dose calculation algorithm present in the TPS, a relatively simple algorithm that does not account for electron transport. XiO has another algorithm available, the Multi-Grid Superposition (MGS) algorithm, which takes electron transport into account and is therefore more accurate in areas with large inhomogeneities such as the lung region 38 , ${ }^{39}$, but is more time consuming. For this study we used the FFTC algorithm for 3D-dose prediction, as routinely applied in our clinic. The MGS algorithm was also used for a better prediction of the dose in the lung region. CT slices were loaded into the TPS, the dose was calculated using inhomogeneity corrections and a 3D-dose view was generated to evaluate the dose distribution over the patient's body.

\subsubsection{MOSFET detector}

MOSFET dosimeters, semiconductor transistors with dimensions of $0.2 \mathrm{~mm}$ by $0.2 \mathrm{~mm}$ by $1 \mathrm{~mm}$ produced by Thomson and Nielsen Electronics Ltd (Ottawa, Canada), were used. For all measurements standard MOSFETs (TN-502RD) were applied, with the bias supply set at standard sensitivity $\left(1 \mathrm{mVcGy}^{-1}\right)$. The TN-RD-50 patient verification system was used for patient measurements. For phantom measurements, which required a lot of repetitive measurements, the MOSFET AutoSense system (TN-RD60 ) developed for on-line radiotherapy was applied in order to shorten measurement 
times, as the control box of the MOSFET system can be read out without entering the treatment room.

Calibration was performed to convert the radiation-induced dosimeter voltage shift to cGy and the calibration coefficient was defined as the ratio of the measured voltage shift of the dosimeter and the actual dose measured with the ionization chamber at the depth of dose maximum $\left(D_{\max }\right)$. A homemade polystyrene phantom, full build-up and reference conditions (6-MV photon beam, $10 \times 10 \mathrm{~cm}^{2}$ field size and $100 \mathrm{~cm} \mathrm{SSD)}$ were used, to obtain maximum accuracy and repeatability. The accelerator output was measured with a calibrated cylindrical ionization chamber (PTW type 31002, Physikalisch-Technische Werkstaetten, Freiburg, Germany), following the Dutch code of practice for the dosimetry of high-energy photon beams ${ }^{40}$. Once calibrated, the dosimeter may be used with or without build-up material when applying appropriate conversion factors.

\subsubsection{TI detector}

For this study, LiF chips type TLD-100 (Harshaw, Solon, USA) were used. Each detector consists of three TLD rods $(1 \mathrm{~mm} \times 1 \mathrm{~mm} \times 6 \mathrm{~mm}$ ) packaged together in a plastic pouch. For all measurements, the readings of the three detectors were averaged.

Before irradiation, the standard annealing procedure was carried out using a Victoreen oven (Model 2600-62, Victoreen Inc., Cleveland, USA). During this process the TLDs were positioned on an aluminum planchet and heated at a temperature of $400^{\circ} \mathrm{C}$ for 1 hour, followed by a lower temperature phase of $105^{\circ} \mathrm{C}$ for 1.5 hours. After annealing, the TLDs were packaged and for each patient twelve packages from the same batch were used, seven for calibration and five for patient measurements.

In order to establish the dose as a function of TLD reading, a calibration was performed using reference conditions. The relationship between TLD response and absorbed dose was expressed by a linear fit for a dose range between 0.2 and $3.0 \mathrm{~Gy}$. One TLD package was read out without receiving radiation to determine the background signal. The detectors were read out with a commercial semiautomatic TLD reader (Harshaw 4000 , Solon, USA). The heating cycle of this oven has two parts, a 5-second pre-heat zone heating the tray to a temperature of $150^{\circ} \mathrm{C}$ and a reading-phase of 10 -seconds using a linear heating rate of $25^{\circ} \mathrm{C} / \mathrm{s}$ followed by a constant temperature plateau of $235^{\circ} \mathrm{C}$. One person performed both the annealing and heating, following strict procedures.

\subsubsection{Phantom measurements}

The accuracy of the central axis Percentage Depth Dose (PDD) data, beam profiles and absolute dose values calculated by the TPS were checked under TBI 
circumstances. The actual dose values were measured under TBI circumstances using an ionization chamber at the center of a polystyrene phantom $(40 \mathrm{~cm} \times 40 \mathrm{~cm} \times 30$ $\mathrm{cm})$. For the PDD measurements the depth varied between 2 and $28 \mathrm{~cm}$. The off-axis dose values were measured at a depth of $10 \mathrm{~cm}$ with an interval of $2 \mathrm{~cm}$ up to off- axis positions of $20 \mathrm{~cm}$, and intervals of $4 \mathrm{~cm}$ for further positions, by shifting the position of the phantom. The actual dose values were compared to the dose values calculated by the TPS for these positions, inside a rectangular phantom of $40 \mathrm{~cm} \times 40 \mathrm{~cm} \times 30 \mathrm{~cm}$.

A conversion factor under TBI conditions was determined to convert entrance and exit in vivo readings, obtained without build up material and using the calibration factor, Fcal ${ }_{(10 \times 10,100)}$, determined under reference conditions $\left(100 \mathrm{~cm} \mathrm{SSD}, 10 \times 10 \mathrm{~cm}^{2}\right.$ field size), to midline dose data under TBI circumstances. To obtain this factor, three calibrated MOSFET detectors were irradiated under TBI conditions, positioned at the entrance and exit point of a full scatter phantom close to the isocentre of the beam. The diameter of the phantom was $10 \mathrm{~cm}, 20 \mathrm{~cm}$ or $30 \mathrm{~cm}$. An ionization chamber (PTW type 31002, Physikalisch Technische Werkstaetten, Freiburg, Germany) was placed at the center of this phantom. A $1.5 \mathrm{~cm}$ thick PMMA screen was positioned between the source and the phantom to bring the position of dose maximum to the surface. An Elekta SL-15i linear accelerator with a 10-MV photon beam was used. MOSFET entrance and exit readings for a field size of $40 \times 40 \mathrm{~cm}^{2}$ at $400 \mathrm{~cm}$ SSD (Rentr (40*40,400) $^{*}$ and Rexit $\left(_{\left(40^{*} 40,400\right)}\right)$ were compared to midline ionization chamber dose values (Dchamber). The factor to convert entrance and exit MOSFET readings to midline dose is called Fconversion. The value of Fconversion was determined for diameters (d) of 10,20 and $30 \mathrm{~cm}$ and dose rates (D) of 100 and $400 \mathrm{MU} / \mathrm{min}$. Fconversion was then calculated as:

$$
\text { F } \text { conversion }_{(\mathrm{d}, \mathrm{D})}=\frac{\mathrm{Dchambe}_{(\mathrm{d}, \text { midline })}}{\left(\operatorname{Rent}_{(\mathrm{d}, 40 \times 40,400)}{ }^{*} \mathrm{~F} \operatorname{cal}_{(10 \times 10,109}+\operatorname{Rexit}_{(\mathrm{d}, 40 \times 40,400)}{ }^{*} \mathrm{~F} \operatorname{cal}_{(10 \times 10,100)}\right) / 2}
$$

Fconversion values were determined in the same way for TLD entrance and exit measurements. Once Fconversion is known, midline dose values (Dmidline) can be obtained from measured entrance and exit MOSFET readings using:

$$
\begin{aligned}
& \text { Dididine }_{(\mathrm{d}, \dot{\mathrm{D}})}= \\
& {\left[\left(\operatorname{Rentr}_{(\mathrm{d}, 40 \times 40,400)}{ }^{*} \mathrm{~F} \operatorname{cal}_{(10 \times 10,100)}+\operatorname{Rexit}_{(\mathrm{d}, 40 \times 40,400)}{ }^{*} \mathrm{Fcal}_{(10 \times 10,100)}\right) / 2\right]{ }^{*} \mathrm{~F} \text { conversion }}
\end{aligned}
$$

In the clinical situation the patient-to-screen distance varies for each part of the body, depending on the position and thickness of the patient. The build up effect of the screen and the influence of varying phantom-screen distance on the entrance and exit dose values was therefore also investigated under TBI conditions. The build up effect was tested by relating the dose values measured with the screen positioned directly in 
contact with the phantom, to the dose values performed without the screen. The influence of the screen-phantom distance on the dose values was investigated by varying the position of the PMMA screen between 0 and $40 \mathrm{~cm}$ distance from the phantom. Both entrance and exit measurements were performed using three MOSFET dosimeters placed at the entrance point and two detectors at the exit point of the beam. For each situation, ten measurements were performed.

\subsubsection{Patient measurements}

Patient measurements were performed to investigate dose homogeneity and the influence of patient position in each individual patient and to compare TPS predicted data with MOSFET and TLD in vivo dose data. Before treatment a total body CT scan was made with the patient in treatment position, i.e. lying in a vaccuum mattress on the right side of the body. Rice bags were positioned around the extremities, to reduce the dose at these positions during irradiation. By performing the CT scan with these rice bags, the dose calculated using the $\mathrm{CT}$ data should be equal to that during the clinical situation and the calculated and measured dose data can then directly be compared. A set of $1 \mathrm{~cm} \mathrm{CT}$ slices was made, from the ankle to the patient's head. The CT images were loaded into our clinical TPS. Dose distributions were normalised to the dose at the prescription point, midline at the position of the umbilicus. Midline dose values were determined at five locations in the patient's body, i.e. (1) jugulum; (2) right lung; (3) umbilicus; (4) upper leg and (5) ankle.

For the treatment, an Elekta SL-15i linear accelerator and a 10-MV photon beam were used, applying the maximum field size of $40 \times 40 \mathrm{~cm}^{2}$, whilst the collimator was rotated over 45 degrees. By positioning the gantry at $274^{\circ}$ and the couch alongside the treatment room wall, an extended source mid-line distance of $400 \mathrm{~cm}$ could be achieved, in order to cover the patient's entire body with the radiation field. Parallel opposed beams were used by delivering each fractional dose in two equal set-ups and switching the patient's position between the two fields. Because the TBI protocol does not require skin sparing, a $1.5 \mathrm{~cm}$ thick PMMA screen was positioned between the source and the patient to raise the surface dose to at least $90 \%$ of the prescribed TBI dose. The number of MUs was calculated using:

$$
\# M U s=\frac{T T D}{O F} *[1+(P D U-20) / 100]
$$

where TTD is the Total Target Dose, OF is the Output Factor of the linear accelerator in cGy per MU at a distance of $400 \mathrm{~cm}$ at a depth of $10 \mathrm{~cm}$ in water, and PDU is the Patient Diameter at the position of the Umbilicus in $\mathrm{cm}$. The number of MUs is prescribed at the patient's midline. The second part of equation 3 accounts for the difference in position between the dose prescription point and OF depth. An increase in depth of $1.0 \mathrm{~cm}$, which corresponds to an increase in patient diameter of $2.0 \mathrm{~cm}$, results in an increase in \# MUs of $2.0 \%$. This equation is similar to using a tissue- 
phantom ratio varying linearly with depth, which is a valid assumption at this extended SSD and relevant depths of 10 to $15 \mathrm{~cm}$. In this equation, 20 is the diameter of the OF phantom in $\mathrm{cm}$. MOSFET and TLD detectors were taped to the patient, with the round epoxy side of the detector towards the skin, at the entrance and exit point at five locations on the patient's body, previously determined during CT scanning. The measured entrance and exit MOSFET and TLD readings were converted to midline dose, using Fconversion, as described earlier. MOSFET and TLD midline dose values were compared to midline dose values calculated by the $\mathrm{XiO}$ treatment planning system, at these five locations.

For a sub-group of patients an additional CT scan was made to investigate the influence of patient positioning on the 3D-dose prediction. Two sets of CT scans were made, one with the patient having the arms crossed before the thorax and the other with one or two arms along the body, depending on the ability of the patient to lay comfortably in such a position. The CT scans were loaded in the TPS and a 3D-dose calculation was made for both positions.

\subsection{RESULTS}

\subsubsection{Phantom measurements}

The accuracy of the TPS along the central axis of the beam at a depth of $10 \mathrm{~cm}$ using TBI conditions was excellent. The output of the accelerator and the absolute dose calculated by the TPS under these circumstances agreed within $1.7 \%$, the calculated value being lower. The PDD calculated by the TPS up to a depth of $28 \mathrm{~cm}$, was within $2.0 \%$ of the actual dose measured with the ionization chamber. The dose profile of the linear accelerator is almost flat; the off-axis dose values were within $2.0 \%$ of the dose on the central axis. These measured dose values, compared to the dose values calculated by the TPS for these positions, agreed within $1.2 \%$, as can be seen in figure 1.

Fconversion, as determined for the MOSFET dosimeters, is $1.12 \pm 0.01,1.08 \pm 0.01$ and $1.06 \pm 0.01$, for phantom diameters of $10 \mathrm{~cm}, 20 \mathrm{~cm}$ and $30 \mathrm{~cm}$, respectively. We interpolated this data using the equation $0.000069 x^{2}-0.00565 x+1.1675\left(R^{2}=1\right)$ to determine the factor for other diameters. The conversion factors to convert TLD entrance and exit values to midline dose values are $1.15 \pm 0.02,1.10 \pm 0.01$ and 1.07 \pm 0.01 , using the equation $0.000112 x^{2}-0.00858 x+1.2248\left(R^{2}=1\right)$ to interpolate the data. Fconversion is independent of dose rate. The results are shown in figure 2. 


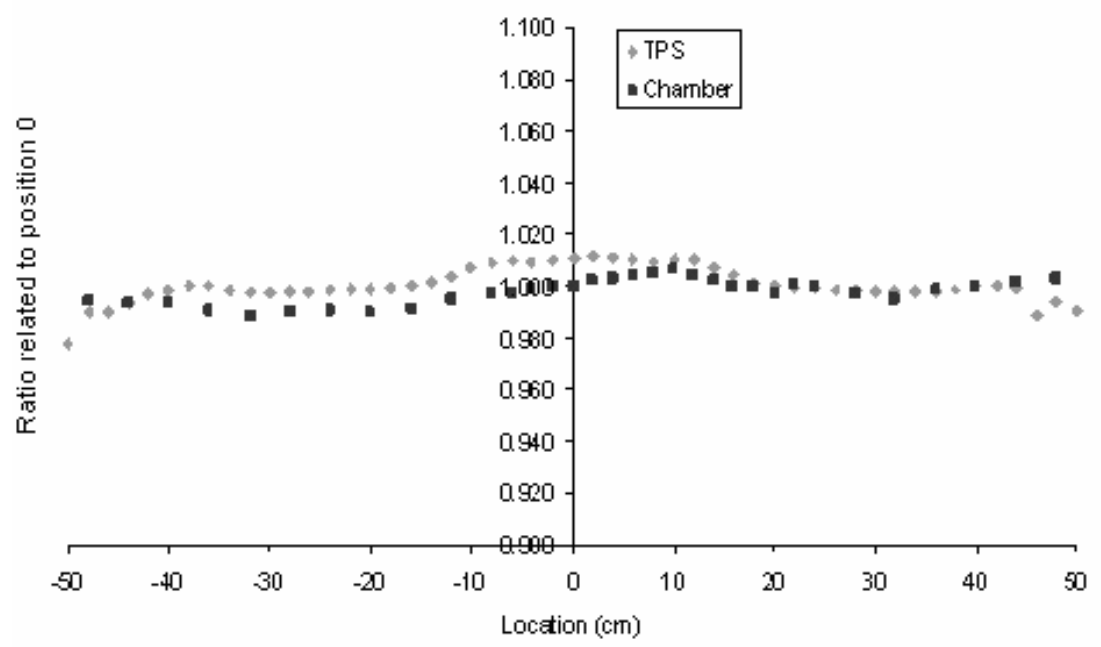

Figure 1: Dose profile of the TBI beam of the linear accelerator at a depth of $10 \mathrm{~cm}, 40 \times 40$ $\mathrm{cm}$ field size and $400 \mathrm{~cm}$ SSD, measured with an ionization chamber and calculated by the Treatment Planning System (TPS).

The ratio of the entrance dose values measured with a MOSFET dosimeter in the surface region (water-equivalent thickness of the MOSFET is $0.8 \mathrm{~mm}$, flat sided), with and without the PMMA screen, was 1.51. The dose in the surface region decreases with increasing phantom-to-screen distance. For distances of 5, 10, 20 and $40 \mathrm{~cm}$ the ratios, normalized to the dose value with the screen at $0 \mathrm{~cm}$ distance, are $0.97,0.94$, 0.92 and 0.83 respectively (figure 3 ).

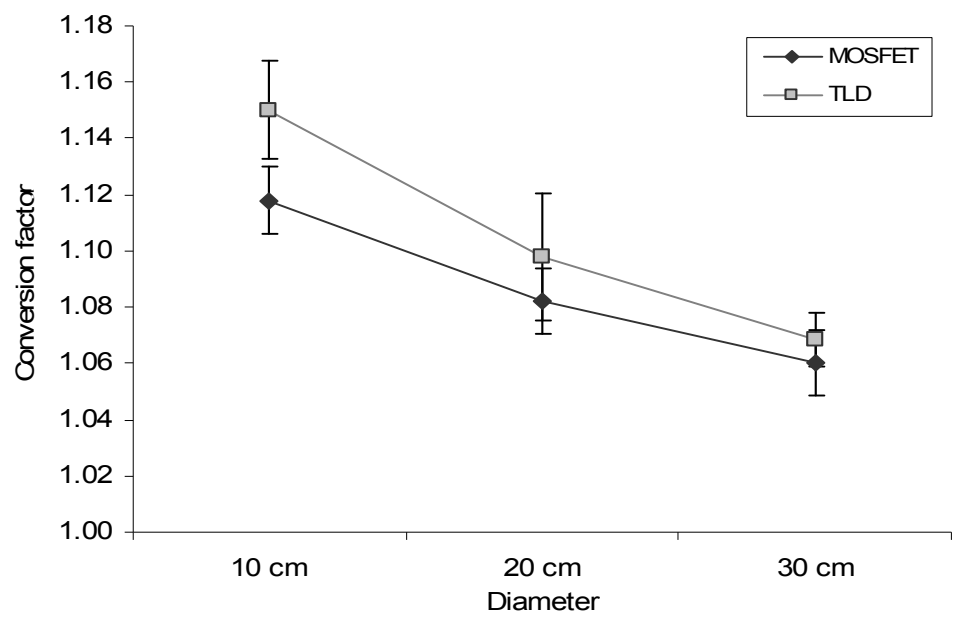

Figure 2: Fconversion, determined in a 10-MV photon beam using a dose rate of $100 \mathrm{MU} / \mathrm{min}$. Phantom diameters ranged between $10 \mathrm{~cm}$ and $30 \mathrm{~cm}$. 


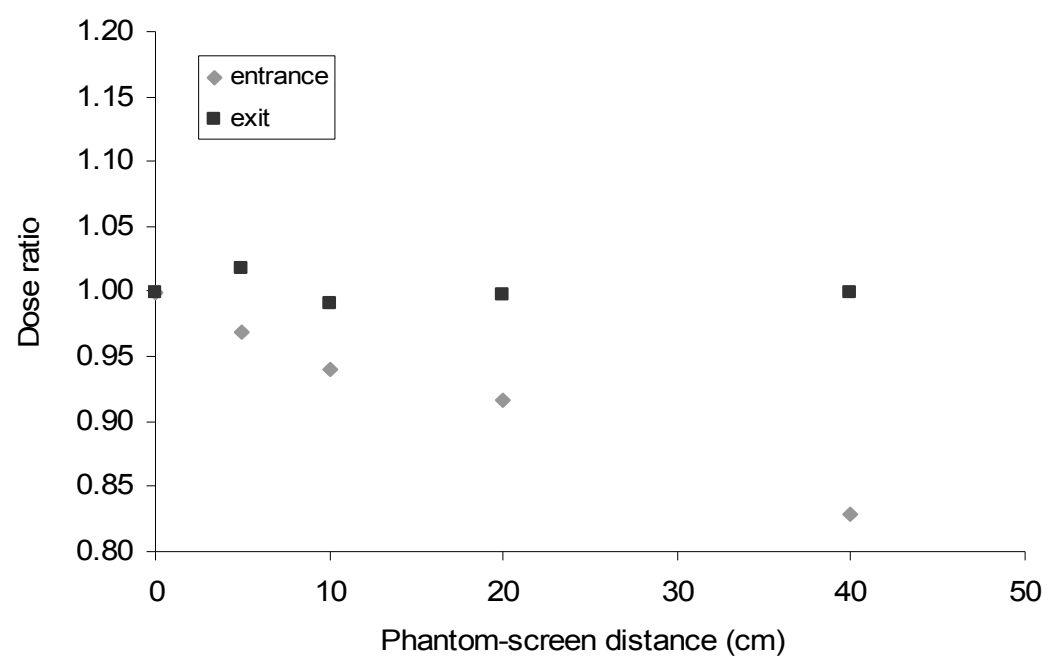

Figure 3: Entrance and exit dose measurements as a function of phantom-screen distance, normalized to $0 \mathrm{~cm}$. Average results of ten measurements are shown for each distance.

\subsubsection{Patient measurements}

Ten patients were included in the clinical study and fifty entrance- and exitmeasurements were performed. A radiation dose of $200 \mathrm{cGy}$ prescribed at the dose prescription point, was given in one single fraction. The PDU, used to calculate the number of MUs, ranged from $24 \mathrm{~cm}$ to $36 \mathrm{~cm}$. MOSFET and TLD measured entrance and exit readings were converted to midline dose using Fconversion and the relevant radiological diameters of the patient taken from the CT-data. The mean difference between the MOSFET and TLD results was $-3.0 \pm 0.2 \%$ (1SD), where the mean MOSFET reading was lower compared to the TLD reading. The maximum deviation was found in the upper leg and the ankles, where a deviation of $-4.2 \%$ and $-4.0 \%$, respectively, was found. The reason might be that the MOSFET and TL detector were taped next to each other at these smaller parts of the body where relatively large dose gradients exist. The mean MOSFET dose values were $0.5 \pm 0.1 \%$ (1SD) lower compared to the TPS dose values, while the TLD results were $2.4 \pm 0.1 \%$ (1SD) higher compared to the TPS data. Part of the observed differences may be due to the somewhat lower dose calculated by the TPS, as observed during the phantom measurements. Mean MOSFET, TLD and TPS results for the five measurement locations are given in table 1. 


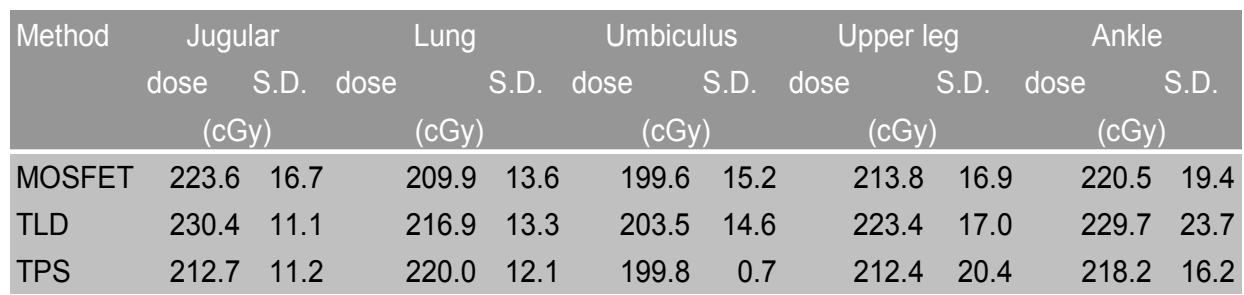

Table 1: Mean MOSFET, TLD and TPS dose values for the five measurement locations. The TPS data were calculated using the MGS algorithm at the position of the lung and the FFTC algorithm for the other locations.

Several authors have shown that the rather simple tissue inhomogeneity correction in the FFTC dose calculation algorithm overestimates the dose in lung dose regions. To quantify the influence of the FFTC algorithm, which is applied routinely in our clinic, on the 3D dose distribution, calculations were also performed using the MGS algorithm present in the TPS 38,39 . Using this algorithm reduced the calculated lung dose by approximately $3 \%$, yielding better agreement between the measured and TPS calculated dose values. For the other, relative homogeneous areas, using the MGS algorithm has no significant advantage, however extends the calculation times significantly. The data, using the MGS algorithm for the lung dose points and the FFTC algorithm for the other locations, are presented in figure 4.

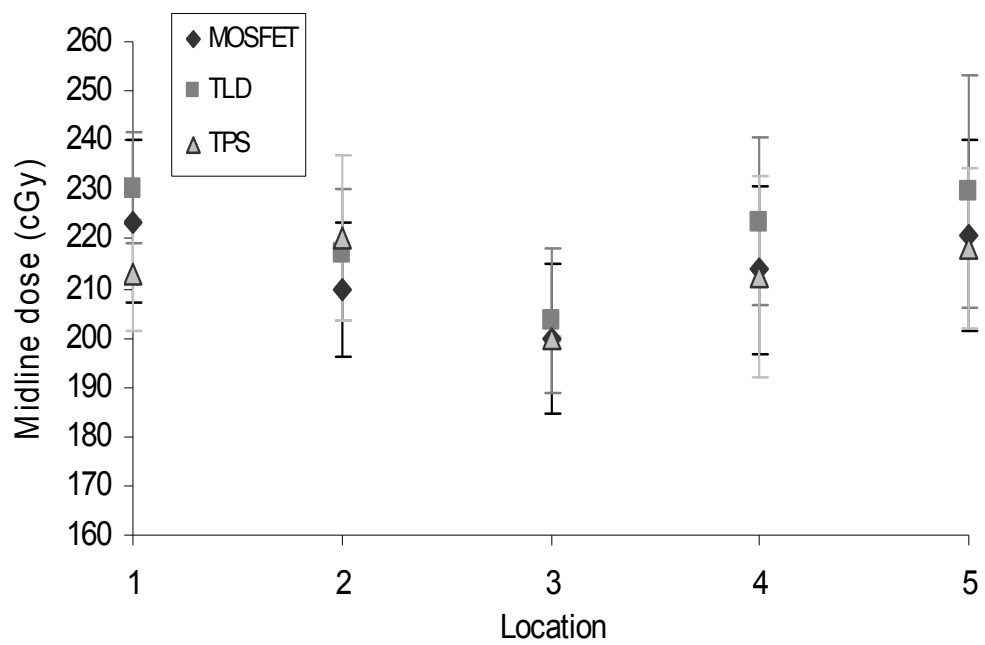

Figure 4: Midline patient dose values measured with MOSFET and thermoluminescence detectors (TLD), and calculated with theTreatment Planning System (TPS). The mean result of 8 patients and the standard deviation are given. The locations 1 to 5 are positioned at the jugulum, right lung, umbilicus, upper leg and ankle, respectively. 
Individual patient measurements demonstrated high dose values, particularly in the extremities, where deviations of $24 \%$, compared to the prescribed dose, were found. The 3D-dose distributions, calculated by the TPS, showed even higher local dose values. To get an impression of the high dose regions in one patient during a TBI treatment, a 3D view is presented in figure $5 \mathrm{a}$, illustrating the areas having $110 \%$ or higher dose values and a local dose of $246.3 \mathrm{cGy}$. Figure $5 \mathrm{~b}$ shows isodoselines in a transversal plane in the pelvic region and figure $5 \mathrm{c}$ isodoselines in a transversal plane in the thoracic region.
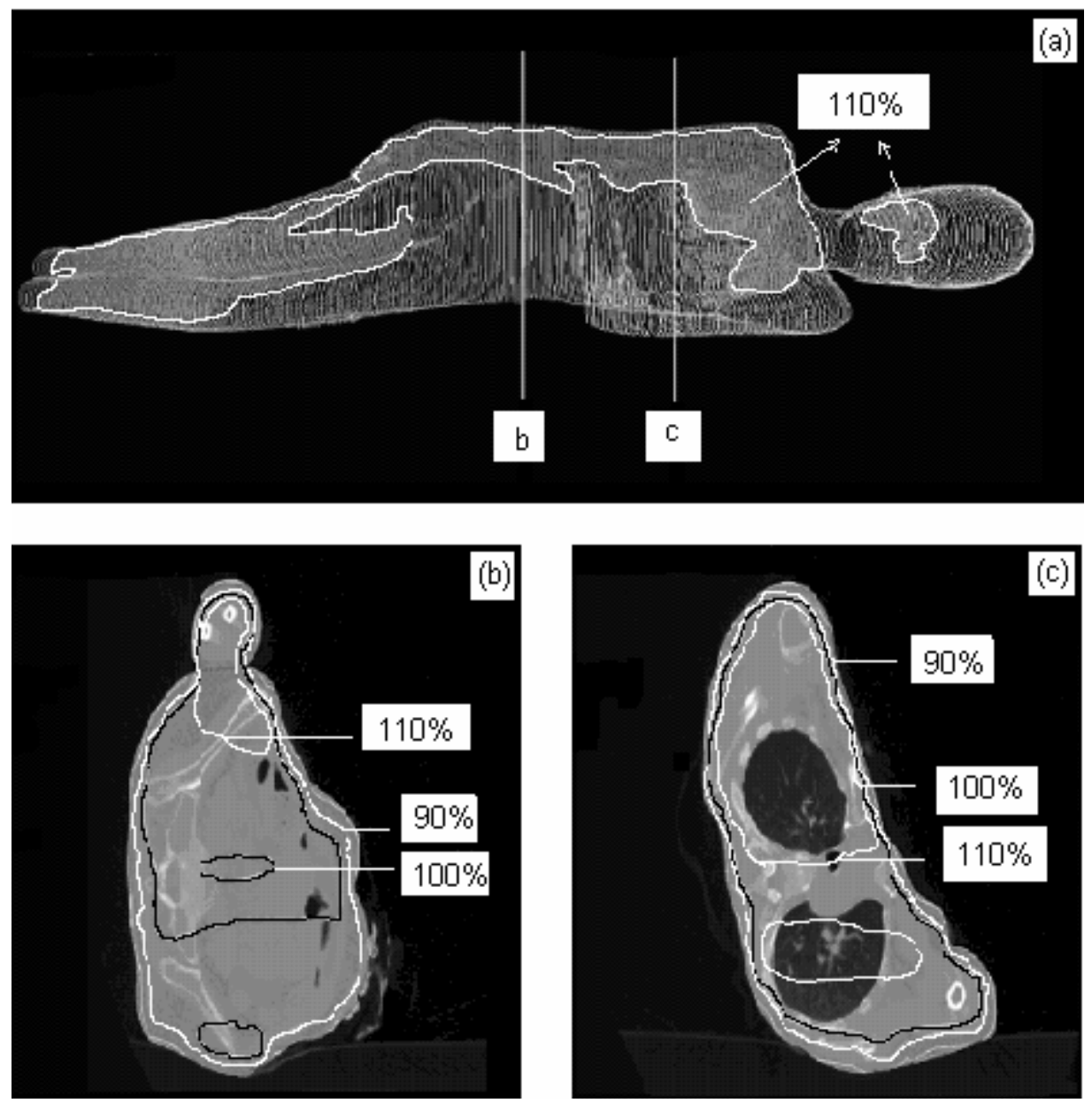

Figure 5a: 3-dimensional (3D)-dose distribution of an individual patient, indicating areas having a dose higher than $110 \%$ (grey area). b: 110\% (white line), 100\% (black line) and 90\% (white line) isodoselines in a transverse plane, in the pelvic region. c: $110 \%$ (white line), $100 \%$ (black line) and $90 \%$ (white line) isodoselines in the thoracic region. 
The influence of patient positioning, investigated by the dose calculation with the patient in two positions, demonstrated that the arms crossed before the thorax gives the opportunity to reduce the higher dose values in the lungs. Such a procedure is, however, not sufficient for high-dose TBI, where the dose in the lung should be reduced by lung blocks. By crossing the arms, in some patients the upper arm is facing the beam and reducing the dose in the areas underneath, while the patient is not capable to bend the arm in front of the thorax. Crossing the arms at a lower place, e.g. in the stomach area, can reduce this inconvenience, nevertheless has the disadvantage of shadowing the belly region. By positioning the arms along the body there is no shadowing of the arms, however the dose in the upper arm will receive higher doses values caused by the smaller diameter. Thereby, most patients indicated that it was not pleasant to lie upon their arm, so in several cases the arm underneath was bend before the patient's body.

\subsection{DISCUSSION AND CONCLUSIONS}

A commercially available TPS is in principle suitable for predicting the dose distribution in a patient under TBI conditions ${ }^{6-9,}{ }^{41}$. In earlier publications, these CT-based plans were mainly used for deriving better dose values in some parts of the body, e.g. by having more accurate information of the patient thickness ${ }^{8}$. CT-based treatment planning for TBI may provide, however, precise and accurate dose calculations for the whole body and allows for the correlation of clinical outcomes with the dose values actually delivered. Dose distributions can in addition be used for evaluating the positioning of the patient. This study demonstrated a large variation in dose. Part of the high-dose values observed for the lungs could be explained by the dose calculation algorithm. Depending on its presence in the TPS, a MGS or comparable advanced dose calculation algorithm is recommended to calculate the dose in the lung region.

Most TBI protocols require a dose homogeneity along the body axis within $\pm 10 \% 1$. Due to the large variation in body thickness and internal tissue heterogeneities, it is difficult to meet this requirement and the use of compensators is necessary. As applied in many centers, we used rice bags as a bolus to reduce the dose in the extremities. In vivo dosimetry and planning system results demonstrated that this rice bags significantly reduced the higher dose regions, however were not sufficient to reduce the dose within the required $10 \%$ dose variation. Preliminary measurements to investigate the use of a Multi Leaf Collimator (MLC) as a compensator, are promising. Figure 6 illustrates an MLC compensator, for the same patient shown in figure 5 . Figure $6 a$ shows the leaf position and figure $6 \mathrm{~b}$ the 3D-dose distribution in the patient, treated with both open and MLC beams using beam weights of 1.00 and 0.12 , respectively. The high dose regions are significantly reduced. Further optimization can be obtained when more MLC segments are used. Simple intensity-modulated radiation therapy 
(IMRT) treatment techniques of TBI may therefore be a good alternative to treat these patients with an optimal dose distribution, as described by Hui et a/42. Patient data resulting from the use of IMRT in TBI treatments using conventional linear accelerators, however, has not been described and is subject of further study in our department.
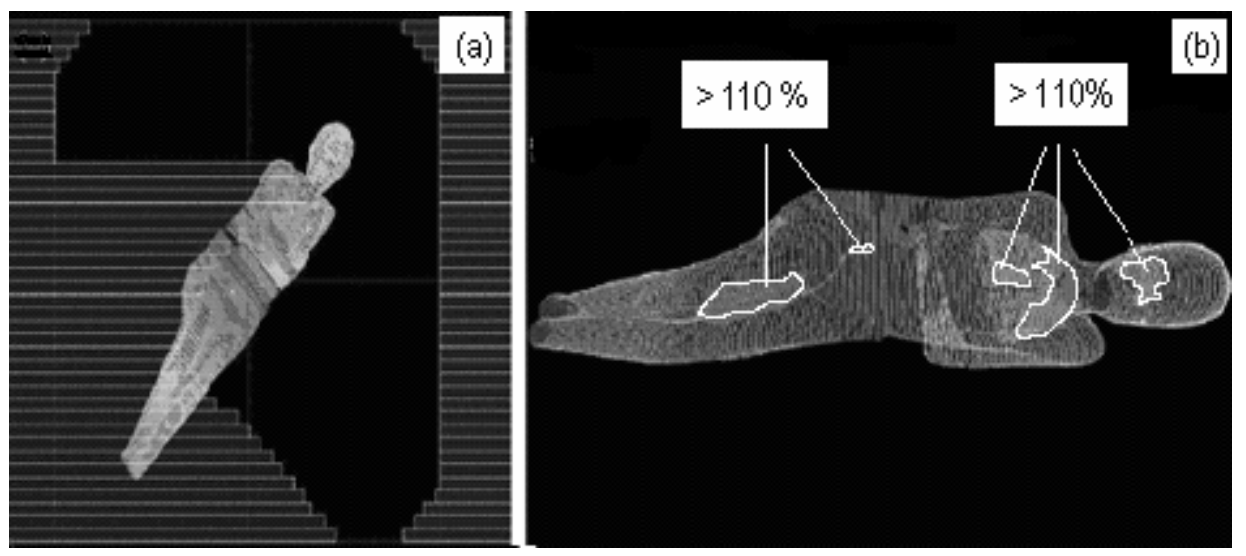

Figure 6: a: A Total Body Irradiation (TBI) beam shaped with a Multi Leaf Collimator (MLC), to reduce the high dose regions in the patient, for the same patient as presented in figure $4 a$. $b$ : The high dose regions visible in figure $4 a$, reduced as a result of using the MLC as compensator.

The influence of patient positioning on the dose values in the patient was also investigated by CT-based treatment planning. The position of the patient, resulting in the most homogeneous dose distribution, depends on the treatment technique that is used (lung blocks, compensators) and on the performance status of the patient. Caution must be used when crossing the arms before the lower thorax/belly, because the dose in the areas underneath decreases and an under-dosage is introduced. The arms along the body resulted in higher dose areas in the arms caused by the smaller diameter, however, this problem can be solved when using an MLC as a compensator to reduce the dose in this area.

To receive an acceptable dose in the surface region of the patient, it is important that there is a short patient-to-screen distance. Increasing the screen-to-phantom distance from $0 \mathrm{~cm}$ to $40 \mathrm{~cm}$, which is the clinical situation for the smaller parts of the body, reduces the dose in the surface region by $17 \%$. This causes an under-dose in the superficial areas because patient immobilization makes it almost impossible to position the patient in close contact with the screen along the body axis.

This study demonstrated that both TLDs and MOSFETs are suitable for in vivo dosimetry under TBI circumstances. The detectors, calibrated under reference conditions, were used for in vivo dose measurements under TBI circumstances and their readings were converted to the midline dose, using a conversion factor. In the 
lung region deviations between in vivo dose values and TPS data were observed. The rather simple tissue inhomogeneity correction algorithm (FFTC algorithm) in our TPS was the main reason for this deviation. The deviation decreased when using a MGS algorithm. The use of the same conversion factor for both the homogeneous and inhomogeneous situation also influenced the accuracy. The radiological diameter was used to convert the measurements and account for the lung inhomogeneity assuming the patient is homogeneous. However, if the dose specification point lies inside the lung, our simple conversion method using equation 2, is no longer valid and may underestimate the dose by about $3 \% 43$.

The overall uncertainty in dose determination using MOSFETs and the calibration methodology as applied in this study, is about $3.5 \%$ (1SD). This uncertainty is determined as the root-sum-square of the individual errors of Fconversion, Fcal and R, which are all estimated to be about $2.0 \%$, given as $1 \mathrm{SD}$. The mean MOSFET reproducibility is $1.8 \%$ (1SD) for readings of $200 \mathrm{mV}, 10$ successive measurements and standard sensitivity of the bias supply. The mean reproducibility of our TLD dose determinations was $3.7 \%$ (1SD) under the same circumstances. In this study we placed three TLDs together and averaged the results, to improve the accuracy.

This study demonstrated that CT-based 3D treatment planning of $\mathrm{TBI}$ is not only mandatory for evaluating the complete total body dose distribution, but could also indicate areas to position dosimeters for in vivo dose verification. Points along the central part of the beam are not representative of the high or low dose regions in a patient. For the evaluation of new TBI techniques such as the use of IMRT, in vivo dosimetry, for instance by using MOSFETs, is strongly recommended.

\section{ACKNOWLEDGEMENTS}

We like to thank André Dekker and André Minken for their advice during this study and Przemyslaw Krasuski for his help during the phantom measurements. 


\section{REFERENCES}

1. Van Dyk J, J. M. Galvin, G. P. Glasgow, E. B. Podgorsak. Physical aspects of total and half body irradiation. Report of Task Group 29, American Association of Physicists in Medicine (AAPM). Vol Report No. 17; 1986.

2. Roncadin $M$, Arcicasa $M$, Bortolus $R$, et al. Feasibility of total body irradiation in chronic lymphocytic leukemia and low-grade non-Hodgkin's lymphomas. Cancer Invest 1991:9:403-407.

3. Blume KG, Kopecky KJ, Henslee-Downey JP, et al. A prospective randomized comparison of total body irradiation-etoposide versus busulfan-cyclophosphamide as preparatory regimens for bone marrow transplantation in patients with leukemia who were not in first remission: a Southwest Oncology Group study. Blood 1993;81:21872193.

4. Sanchez-Nieto B, Sanchez-Doblado F, Terron JA. A CT-aided PC-based physical treatment planning of TBI: a method for dose calculation. Radiother Oncol 1997;42:77-85.

5. Planskoy B, Tapper PD, Bedford AM, et al. Physical aspects of total-body irradiation at the Middlesex Hospital (UCL group of hospitals), London 1988-1993: II. In vivo planning and dosimetry. Phys Med Biol 1996;41:2327-2343.

6. Planskoy B, Bedford AM, Davis FM, et al. Physical aspects of total-body irradiation at the Middlesex Hospital (UCL group of hospitals), London 1988-1993: I. Phantom measurements and planning methods. Phys Med Biol 1996;41:2307-2326.

7. Mangili $\mathrm{P}$, Fiorino $\mathrm{C}$, Rosso A, et al. In-vivo dosimetry by diode semiconductors in combination with portal films during TBI: reporting a 5-year clinical experience. Radiother Oncol 1999;52:269-276.

8. Hui SK, Das RK, Thomadsen B, et al. CT-based analysis of dose homogeneity in total body irradiation using lateral beam. J Appl Clin Med Phys 2004;5:71-79.

9. Abraham D, Colussi V, Shina $D$, et al. TBI treatment planning using the ADAC pinnacle treatment planning system. Med Dosim 2000;25:219-224.

10. Ribas M, Jornet N, Eudaldo T, et al. Midplane dose determination during total body irradiation using in vivo dosimetry. Radiother Oncol 1998;49:91-98.

11. Duch MA, Ginjaume M, Chakkor $\mathrm{H}$, et al. Thermoluminescence dosimetry applied to in vivo dose measurements for total body irradiation techniques. Radiother Oncol 1998;47:319-324.

12. Pacyna LG, Darby M, Prado K. Use of thermoluminescent dosimetry to verify dose compensation in total body irradiation. Med Dosim 1997;22:319-324.

13. Sanchez-Doblado F, Terron JA, Sanchez-Nieto B, et al. Verification of an on line in vivo semiconductor dosimetry system for TBI with two TLD procedures. Radiother Oncol 1995;34:73-77.

14. Palkoskova $\mathrm{P}$, Hlavata $\mathrm{H}$, Dvorak $\mathrm{P}$, et al. In vivo thermoluminescence dosimetry for total body irradiation. Radiat Prot Dosimetry 2002;101:597-599.

15. Soubra M, Cygler J, Mackay G. Evaluation of a dual bias dual metal oxide-silicon semiconductor field effect transistor detector as radiation dosimeter. Med Phys 1994;21:567-572. 
16. Ramani R, Russell S, O'Brien P. Clinical dosimetry using MOSFETs. Int J Radiat Oncol Biol Phys 1997;37:959-964.

17. Butson MJ, Rozenfeld A, Mathur JN, et al. A new radiotherapy surface dose detector:the MOSFET. Med Phys 1996;23:655-658.

18. Francescon $\mathrm{P}$, Cora $\mathrm{S}$, Cavedon $\mathrm{C}$, et al. Use of a new type of radiochromic film, a new parallel-plate micro-chamber, MOSFETs, and TLD 800 microcubes in the dosimetry of small beams. Med Phys 1998;25:503-511.

19. Rosenfeld AB. MOSFET dosimetry on modern radiation oncology modalities. Radiat Prot Dosimetry 2002;101:393-398.

20. Scalchi P, Francescon P, Rajaguru P. Characterization of a new MOSFET detector configuration for in vivo skin dosimetry. Med Phys 2005;32:1571-1578.

21. Rowbottoma CG, Jaffray DA. Characteristics and performance of a micro-MOSFET: an "imageable" dosimeter for image-guided radiotherapy. Med Phys 2004;31:609615.

22. Ramaseshan R, Kohli KS, Zhang TJ, et al. Performance characteristics of a microMOSFET as an in vivo dosimeter in radiation therapy. Phys Med Biol 2004:49:4031-4048.

23. Panettieri V, Duch MA, Jornet N, et al. Monte Carlo simulation of MOSFET detectors for high-energy photon beams using the PENELOPE code. Phys Med Biol 2007;52:303-316.

24. Bharanidharan G, Manigandan D, Devan K, et al. Characterization of responses and comparison of calibration factor for commercial MOSFET detectors. Med Dosim 2005;30:213-218.

25. Bloemen-van Gurp EJ, Minken AW, Mijnheer BJ, et al. Clinical implementation of MOSFET detectors for dosimetry in electron beams. Radiother Oncol 2006;80:288295.

26. Kohno R, Nishio T, Miyagishi T, et al. Experimental evaluation of a MOSFET dosimeter for proton dose measurements. Phys Med Biol 2006;51:6077-6086.

27. Bloemen-van Gurp E, du Bois W, Visser P, et al. Clinical dosimetry with MOSFET dosimeters to determine the dose along the field junction in a split beam technique. Radiother Oncol 2003;67:351-357.

28. Chuang CF, Verhey LJ, Xia P. Investigation of the use of MOSFET for clinical IMRT dosimetric verification. Med Phys 2002;29:1109-1115.

29. Marcie S, Charpiot E, Bensadoun RJ, et al. In vivo measurements with MOSFET detectors in oropharynx and nasopharynx intensity-modulated radiation therapy. Int $J$ Radiat Oncol Biol Phys 2005;61:1603-1606.

30. Zilio VO, Joneja OP, Popowski Y, et al. Absolute depth-dose-rate measurements for an 1921r HDR brachytherapy source in water using MOSFET detectors. Med Phys 2006;33:1532-1539.

31. Cygler JE, Saoudi A, Perry G, et al. Feasibility study of using MOSFET detectors for in vivo dosimetry during permanent low-dose-rate prostate implants. Radiother Oncol 2006;80:296-301.

32. Wang $\mathrm{B}, \mathrm{Kim} \mathrm{CH}$, Xua XG. Monte Carlo modeling of a high-sensitivity MOSFET dosimeter for low- and medium-energy photon sources. Med Phys 2004;31:10031008. 
33. Ciocca M, Piazzi V, Lazzari $\mathrm{R}$, et al. Real-time in vivo dosimetry using micro-MOSFET detectors during intraoperative electron beam radiation therapy in early-stage breast cancer. Radiother Oncol 2006;78:213-216.

34. Consorti $R$, Petrucci $A$, Fortunato $F$, et al. In vivo dosimetry with MOSFETs: dosimetric characterization and first clinical results in intraoperative radiotherapy. Int $J$ Radiat Oncol Biol Phys 2005;63:952-960.

35. Jornet N, Carrasco P, Jurado D, et al. Comparison study of MOSFET detectors and diodes for entrance in vivo dosimetry in 18 MV x-ray beams. Med Phys 2004;31:25342542.

36. Lavallee MC, Gingras L, Beaulieu L. Energy and integrated dose dependence of MOSFET dosimeter sensitivity for irradiation energies between $30 \mathrm{kV}$ and 60Co. Med Phys 2006;33:3683-3689.

37. Scalchi P, Francescon P. Calibration of a mosfet detection system for 6-MV in vivo dosimetry. Int J Radiat Oncol Biol Phys 1998;40:987-993.

38. Garcia-Vicente F, Minambres A, Jerez I, et al. Experimental validation tests of fast Fourier transform convolution and multigrid superposition algorithms for dose calculation in low-density media. Radiother Oncol 2003;67:239-249.

39. Miften M, Wiesmeyer M, Monthofer S, et al. Implementation of FFT convolution and multigrid superposition models in the FOCUS RTP system. Phys Med Biol 2000;45:817-833.

40. Mijnheer B, Wittkämper F, Aalbers A, et al. Consistency and simplicity in the determination of absorbed dose to water in high-energy beams: a new code of practice. Ratiother. Oncol. 1986;7:371-384.

41. Kirby TH, Hanson WF, Cates DA. Verification of total body photon irradiation dosimetry techniques. Med Phys 1988;15:364-369.

42. Hui SK, Kapatoes J, Fowler J, et al. Feasibility study of helical tomotherapy for total body or total marrow irradiation. Med Phys 2005;32:3214-3224.

43. Obcemea CH, Rice RK, Mijnheer BJ, et al. Three-dimensional dose distribution of total body irradiation by a dual source total body irradiator. Int J Radiat Oncol Biol Phys 1992;24:789-793. 


\section{In vivo dosimetry in brachytherapy and}

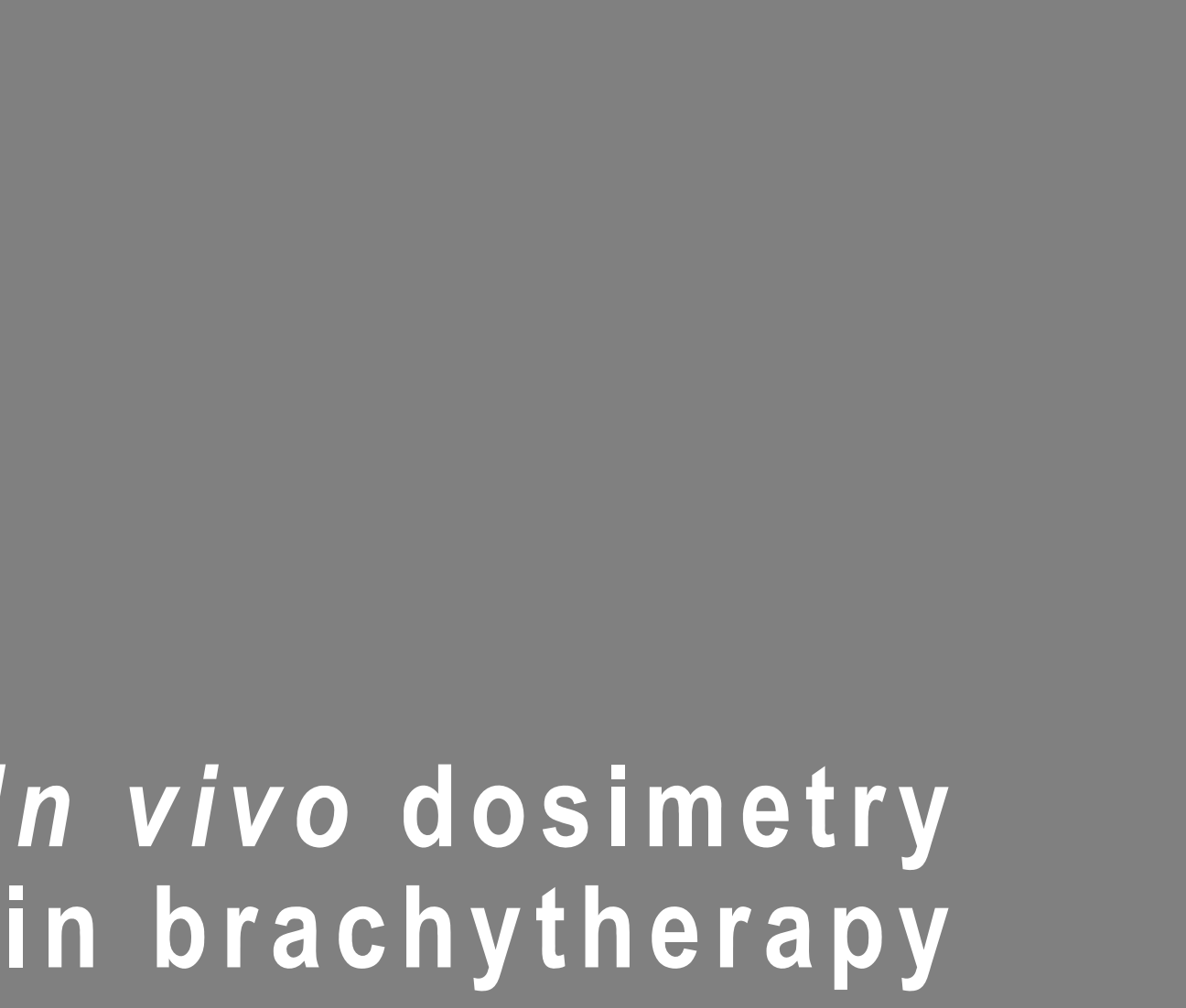





\title{
C H A P T E R
}

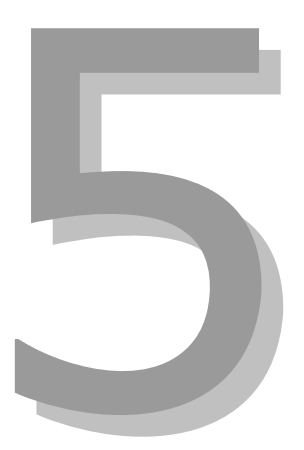

\section{In vivo dosimetry using a linear MOSFET-array dosimeter to determine the urethra dose in ${ }^{125} \mid$ permanent prostate implants}

Int. J. Radiation Oncology Biol. Phys., Vol.73, No. 1, pp. 314-321, 2009

\author{
Esther J. Bloemen-van Gurp \\ Lars H.P. Murrer \\ Björk K.C. Haanstra \\ Francis C.J.M. van Gils \\ Andre L.A.J. Dekker \\ Ben J. Mijnheer \\ Philippe Lambin
}




\section{ABSTRACT}

Purpose: In vivo dosimetry during brachytherapy of the prostate applying ${ }^{125}$ seeds is quite challenging, due to the high dose gradients and low photon energies involved. We present the results of a study using MOSFET-dosimeters to evaluate the dose in the urethra after a permanent prostate implantation procedure.

Methods and materials: Phantom measurements were carried out to validate the measurement technique, to determine the measurement accuracy and to define action levels for clinical measurements. Patient measurements were performed with a MOSFET-array in the urinary catheter directly after the implantation procedure. A CTscan was made and dose values, calculated by the treatment planning system, were compared to in vivo dose values measured with MOSFET-dosimeters.

Results: Corrections for temperature dependence of the MOSFET-array response and photon attenuation in the catheter on the in vivo dose values are necessary. The overall uncertainty in the measurement procedure, determined in a simulation experiment, is $8.0 \%$ (1SD). In vivo dose values were obtained for 17 patients. In the high dose region (>100 Gy) calculated and measured dose values agreed within $1.7 \%$ $\pm 10.7 \%$ (1SD). In the low dose region outside the prostate (<100 Gy) larger deviations occurred.

Conclusions: MOSFET-detectors are suitable for in vivo dosimetry during ${ }^{125}$ brachytherapy of prostate cancer. An action level of $\pm 16 \%$ (2 SD) for detection of errors in the implantation procedure is achievable after validation of the detector system and measurement conditions. 


\subsection{INTRODUCTION}

Brachytherapy using transperineal interstitial permanent prostate implants (TIPPB) addresses a highly conformal dose to the tumor volume. Uncertainties in seed positioning during the implantation procedure, errors in needle positioning, migration of seeds or deviations in seed activity, however, may cause deviations from the prescribed dose. Several studies evaluated the dose in the prostate and organs at risk (OARs) after TIPPB, using a post-implant CT-scan and the treatment planning system (TPS) to calculate the absorbed dose ${ }^{1}$. Using this method, errors in dose calculation, such as inter-seed attenuation, compensation for tissue inhomogeneities and errors in seed activity are not easy to detect-4. In addition, seeds with a short inter-seed distance are difficult to recognize on the CT-image. Inaccuracies in seed registration will result in inaccurate dose information. In vivo dosimetry may provide useful information about the actual dose in the urethra ${ }^{5}$. It will be useful to evaluate the implantation technique, including the accuracy of the TPS and will give us the ability to link dose distributions in OARs to clinical side effects. Nevertheless, in vivo dosimetry during brachytherapy is quite a challenge, due to the high dose gradients and the low energy of the photons emitted by the seeds.

The aim of this study is to validate the new linear 5-MOSFET-array dosimeter for dose verification after permanent ${ }^{125}$ prostate implants, in order to evaluate the dose in the urethra.

\subsection{MATERIALS AND METHODS}

125 brachytherapy sources (Intersource ${ }^{125}$, International Brachytherapy Inc) with a mean nominal activity of $0.6 \mathrm{U}(0.5 \mathrm{mCi})$ were used. The activity of each seed in a batch is stated to be within $\pm 4 \%$ of the mean nominal activity provided by the manufacturer.

Our clinically applied TPS (PSID 4.1, International Brachytherapy), designed for realtime dynamic ultrasound-guided radiation therapy of prostate cancer, was used for dose calculation. This system complies with the AAPM TG-43 recommendations ${ }^{6}$. Patient planning is based on the criteria that the $145 \mathrm{~Gy}$ isodose line covers the prostate volume, the urethra dose does not exceed $200 \mathrm{~Gy}$ and the medium rectum volume exceeding $100 \mathrm{~Gy}$ should be less than $8 \mathrm{cc}^{7}$.

For dose measurements, a high-sensitivity MOSFET-array (Linear-5ive-array TN502LA5, Best Medical, Canada) at high-sensitivity bias setting was used. The array contains five MOSFET dosimeters, which are $4 \mathrm{~mm}$ long, $1.8 \mathrm{~mm}$ wide and $1.3 \mathrm{~mm}$ thick, all fixated on a $46 \mathrm{~cm}$ flexible cable, with $2 \mathrm{~cm}$ inter-MOSFET-spacing. The 
Mobile MOSFET Dose Verification System (TN-RD-70W) of the same company was used for on-line dose readout on a remote-PC.

\subsubsection{Phantom measurements}

\subsubsection{Angular dependence of the MOSFET-array response}

Studies performed with single dosimeters ${ }^{5,8}$ and with the linear-array at ${ }^{60} \mathrm{Co}$ energies 9 , demonstrated a small angular dependence. To test the angular response of the linearMOSFET array for ${ }^{125}$ sources, the detector was placed at the center of an in-house made PMMA phantom (figure 1a). Three strengths, each containing of three seeds, were placed at $0.5 \mathrm{~cm}, 1 \mathrm{~cm}$ and $1.5 \mathrm{~cm}$ distance from the detector, in order to obtain higher dose-rates and more accurate measurements. The direction from the MOSFET to the top of the black epoxy bulb of the detector was defined as 0 degrees. The angle of the detector was varied in steps of $30^{\circ}$ from $0^{\circ}$ to $330^{\circ}$. For each angle 5 measurements were obtained and the results were averaged. The result of each angle was related to the mean result of all angles.

\subsubsection{Calibration of the detector}

A calibration coefficient was determined for each MOSFET of the linear-array as the ratio of the measured voltage shift and the actual dose. An in-house made PMMA calibration phantom $(8 \mathrm{~cm} \times 8 \mathrm{~cm} \times 15 \mathrm{~cm})$ was used, where the MOSFET-array was placed at the center and 12 seeds were positioned circularly around the detector (figure $1 \mathrm{~b}$ ). Each MOSFET was placed in the plane of the seeds, 5 measurements were performed for each MOSFET and the results were averaged and compared to the calculated dose values.

\subsubsection{Absorption of the catheter material}

The in vivo measurements are performed with the MOSFET-array placed in a catheter. The TPS assumes all material surrounding the seeds is water i.e. the influence of catheter absorption on the dose distribution is not included. To test the difference in absorption between water and the catheter material, we performed a set of phantom measurements. An in-house made PMMA phantom, similar to the calibration phantom was used, however with a larger hole in the center of the phantom allowing for catheter placement, and a second ring containing 12 holes for seed placement at $2 \mathrm{~cm}$ distance from the detector in order to approach clinical dose-rates. A Foley-catheter, a silicone 2-way catheter with an outside diameter of $6 \mathrm{~mm}$ (18 french) and a length of $40 \mathrm{~cm}$ (Ref 170605, Teleflex Medical B.V) was positioned in the center of the phantom. The MOSFET-array was placed in the catheter and 24 strands, each containing 3 seeds, were placed around the detector. The phantom was placed in a water tank, in order to 
fill the space between the MOSFET and catheter material with water (figure 1c). The position of the array was determined accurately $( \pm 0.5 \mathrm{~mm})$, having MOSFET 2 positioned in the plane of the center of the strands. The MOSFET-position was related to the marks on the flexible cable of the array, relative to the top of the phantom, and defined as MOSFET-position 1 (MP1). Five measurements were performed. To investigate the absorption of the catheter material in case the radiation passes the catheter under an oblique angle, the MOSFET-array was pulled down along the catheter with a distance of $8.8 \mathrm{~mm}$ (MP2), $17.6 \mathrm{~mm}$ (MP3), $25.1 \mathrm{~mm}$ (MP4), $33.9 \mathrm{~mm}$ (MP5) and $42.7 \mathrm{~mm}$ (MP6), again by using the marks on the flexible cable in relation to the top of the phantom. For each position 5 measurements were performed.

The results were related to an equal set of measurements in the same phantom positioned in a water tank, however with water occupying the space where the catheter material was previously. The results are presented as the ratio of the MOSFET-reading attenuated by catheter material compared to the attenuation of the same layer of water.

\subsubsection{Temperature dependence of the MOSFET-array response}

Ramaseshan et al. ${ }^{13}$ described the temperature response of micro-MOSFETs, demonstrating a $0.5 \%$ variation when increasing the temperature from $20^{\circ}$ to $40{ }^{\circ} \mathrm{C}$. The temperature dependence of the new MOSFET-array was not yet described. To test the variation in MOSFET-array sensitivity due to temperature changes, the largehole phantom (without the catheter material) and 12 single seeds were used. The temperature sensor was placed inside the hole close to the MOSFET-array without shielding the detector from the radiation. The phantom was positioned into a water tank and the water temperature was varied between $19^{\circ} \mathrm{C}$ and $40^{\circ} \mathrm{C}$. MOSFET 3 was positioned in the plane of the seeds. Measurement times of 10 minutes were used. The temperature was determined at the start and the end of the measurement, and the mean temperature was used for the results. The results were related to initial calibration conditions at room temperature $\left(20^{\circ} \mathrm{C}\right)$. 

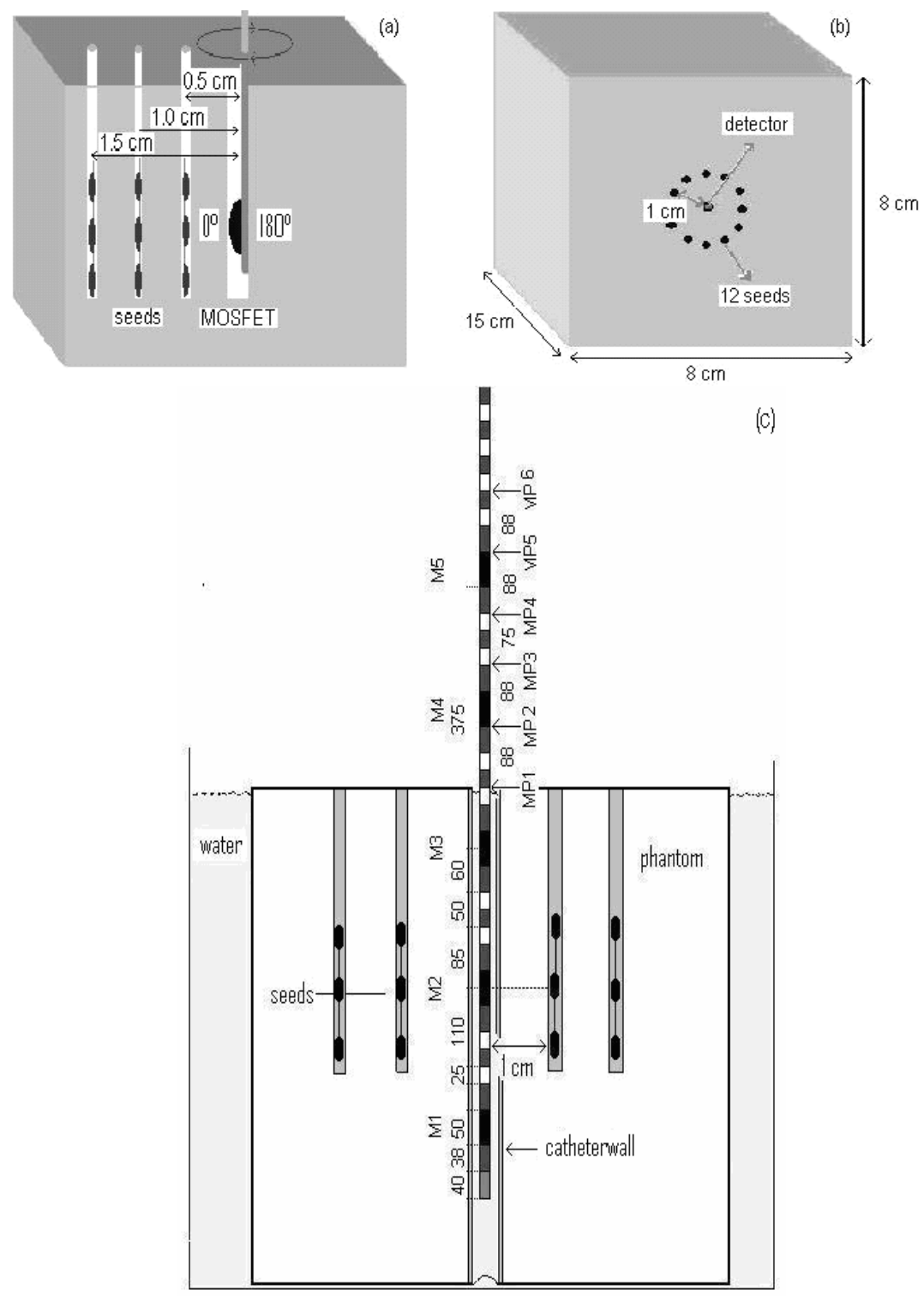

Figure 1: Phantoms used to investigate the response of the linear-MOSFET array. a: Phantom to test the angular response. Seeds were placed at $0.5 \mathrm{~cm}, 1 \mathrm{~cm}$ and $1.5 \mathrm{~cm}$ distance from the detector. The black epoxy bulb of the detector was defined as $0^{\circ}$, the angle of the detector was varied in steps of $30^{\circ}$ from $0^{\circ}$ to $330^{\circ}$. b: Phantom for calibration of the MOSFET-array to convert the radiation induced voltage shift into dose. The linear MOSFET-array is inserted in the central hole, in the plane of the seeds. c: Set-up to investigate the absorption by the catheter material. The phantom $(8 \mathrm{~cm} \times 8 \mathrm{~cm} \times 15 \mathrm{~cm})$ is placed in a water tank, a catheter placed at the centre of the phantom. The results of the 5 MOSFETs (M1-M5) and six MOSFET-array positions (MP1-MP6) were related to an equivalent set of measurements without the catheter material. 
(d)
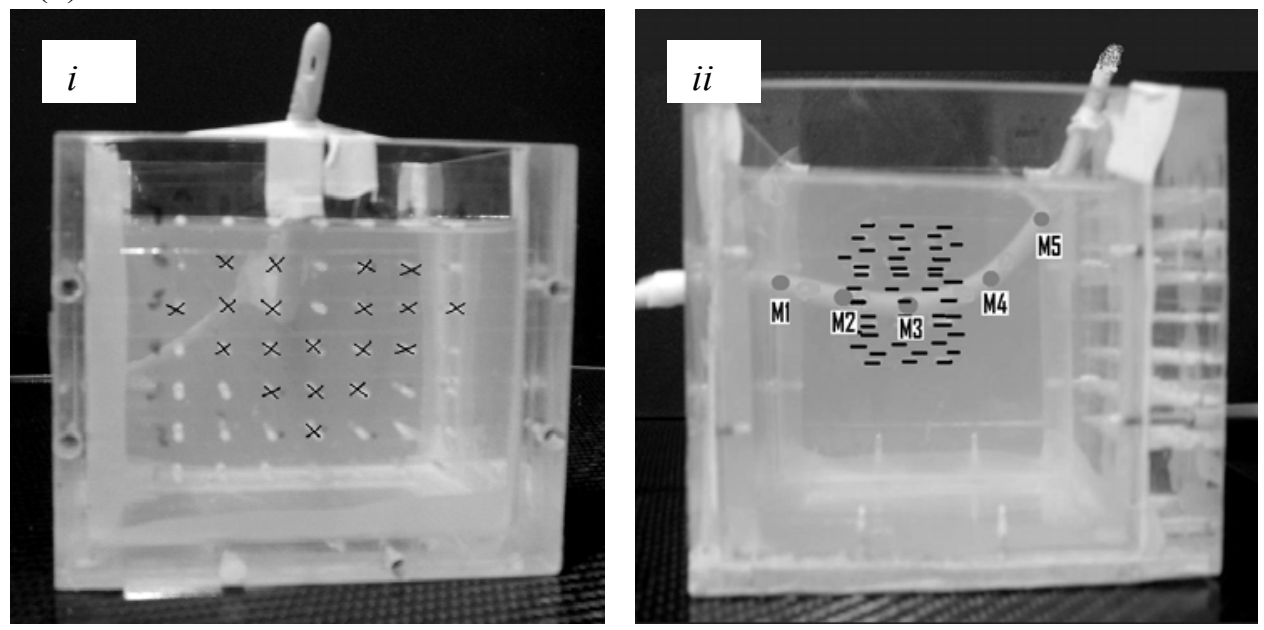

Figure $1 \mathrm{~d}$ : Set-up to test the total measurement uncertainty, presented as a frontal view (i) and a lateral view (ii) of the phantom. The marks, illustrated in the frontal view, present the holes that were used for seed implantation. The lateral view presents the seed distribution, the urinary catheter and the MOSFET-array positions in the catheter.

\subsubsection{Total measurement uncertainty}

The reproducibility of the MOSFET-detector depends on the energy of the radiation and the integrated dose measured by the MOSFET detector. Lavallee et. al. ${ }^{10}$ reported uncertainties of about $6 \%$ (1 SD) using an orthovoltage beam with a mean energy of 30 $\mathrm{keV}$ and dose values of about $55 \mathrm{cGy}$. During the clinical measurements, the uncertainty may be even larger due to the lower signal and positional inaccuracies of the detector. To estimate the total uncertainty, a simulation of a complete treatment was performed. A tank, containing a urinary catheter, was filled with gelatin. Needles were inserted through the holes in the wall of the phantom. We planned a needle distribution, mimicking a clinical plan, using 19 needles and 57 seeds. After the placement of the seeds the MOSFET-array was placed in the urinary catheter and dose values were obtained (position 1). Five measurements of 10 minutes were performed and the results were averaged. After the measurements the MOSFET-array was pulled out over a distance of $1 \mathrm{~cm}$, in order to obtain more dose information along the catheter (position 2). Again 5 measurements were performed. The in vivo dose values of both positions were extrapolated to the accumulated dose values, using the half-life $\left(\mathrm{t}_{1 / 2}\right)$ of 125$)$ :

$\mathrm{D}=\mathrm{IDR} *\langle\mathrm{t}\rangle$ and $\langle t\rangle=\left(t_{1 / 2}\right) / \ln 2$ 
where IDR is the Initial Dose Rate and $t_{1 / 2}$ is 59.4 days. The measurement set-up is shown in figure 1d. Figure 1di presents a frontal view of the phantom and the template. The marks illustrate the holes that were used for seed implantation. Figure 1dii presents a lateral view of the phantom, showing the seed distribution, the urinary catheter in the gelatin and the MOSFET-positions in the catheter.

After the in vivo measurements, a dummy MOSFET-array having radio-opaque markers at each MOSFET-detector position was placed in the catheter. The phantom was placed in a CT-scanner and CT-slices of $1 \mathrm{~mm}$ thickness and $1 \mathrm{~mm}$ separation were acquired for both MOSFET positions 1 and 2 . The CT-scans were loaded into the TPS and the measured extrapolated dose results were compared to the TPS calculated dose values.

\subsubsection{Patient measurements}

In vivo dose measurements were performed in 17 patients. Directly after the implantation, the patient was transported to the recovery. A Y-connector was inserted between the catheter and the urine bag and a sterilized high-sensitivity linear-array was placed into the catheter. The end of the array was positioned at the top of the catheter. The position of the array was fixed using a rubber wedge, which also prevented urine leakage from the catheter. Measurements were performed for one hour, with automatic read-out every 5 minutes. Constant dose-rate was assumed during the interval, in light of the half-life of ${ }^{125}$. After the dose measurements, the array was removed from the catheter and the patient was transported to a CT-scanner. In the scanner room, a sterilized dummy linear-array was positioned to the top of the catheter and again fixed with the rubber wedge. A CT-scan was made, using $1 \mathrm{~mm}$ slicethickness and slice-separation from the bladder dome up to the perineum. The CTslices were then loaded into our TPS. In vivo dose data and calculated dose values were compared.

\subsection{RESULTS}

\subsubsection{Phantom measurements}

\subsubsection{Angular dependence of the MOSFET-array response}

The results of the measurements of the angular dependence of the detector response, related to the mean result of all angles, are given in figure 2. A deviation of $3.1 \%$ $( \pm 0.51 \%, 1 \mathrm{SD})$ at $0^{\circ}$ was observed, probably due to the shape of the epoxy layer that imbeds the MOSFET. The deviations at other angles were within the observed deviation at $0^{\circ}$. The results are comparable to the results of other studies ${ }^{5,8,9}$. 


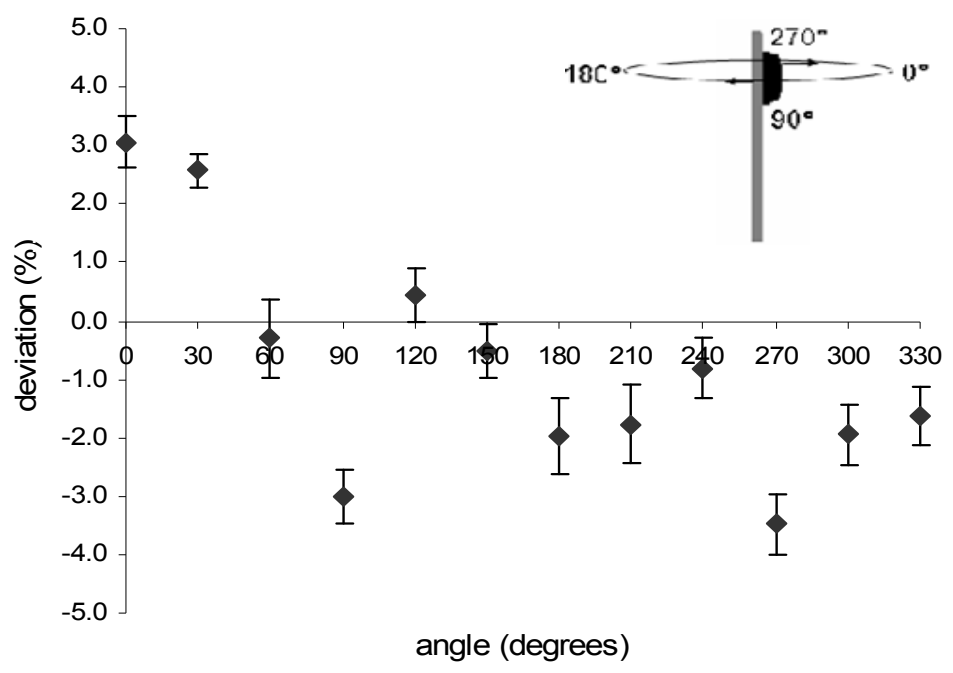

Figure 2: Angular dependence of the MOSFET array response for ${ }^{125}$ photons. The result of each angle was related to the mean value of all angles.

\subsubsection{Calibration of the detector}

The sensitivity of the individual MOSFETs of the MOSFET-array, ranged between 15.2 $\mathrm{mV} / \mathrm{cGy}$ and $16.5 \mathrm{mV} / \mathrm{cGy}$ resulting in an average calibration coefficient of the MOSFETs of $0.06 \mathrm{cGy} / \mathrm{mV}$ with an uncertainty of $\pm 6 \%$ (1SD). The results are consistent with the values provided by the manufacturer for a random batch of MOSFET-arrays. The response of the detector was linear within $1 \%$ for dose values ranging from $0.4 \mathrm{cGy}$ to $4 \mathrm{cGy}$. For values below $0.05 \mathrm{cGy}$, the background noise exceeds the MOSFET-reading indicating that dose values below $0.05 \mathrm{cGy}$ are not reliable.

\subsubsection{Absorption of the catheter material}

The degree of absorption by the catheter material depends on the angle of incidence of the radiation on the catheter. If the MOSFET is positioned in or very close to the plane of the seeds (MP1, MP2 and MP3), the effect of the absorption of the catheter material compared to water is almost constant. When the radiation passes the catheter obliquely, the absorption increases. The additional absorption measured by the MOSFET positioned in the catheter relative to the measurements in water is for MP1, MP2 and MP3 on average $9.1 \%$. For MP4, MP5 and MP6 the absorption is $21.8 \%$, $24.8 \%$ and $37.6 \%$, respectively. A correction for catheter attenuation is therefore 
required. In practice it is difficult to perform such a correction because the dose will be influenced by the number of seeds at different distances from the measuring point. We used an approach where we linked the distance between the MOSFET and the seeds, and thus the angle of incidence, to the IDR. Figure 3 illustrates the deviation, caused by the absorption of the catheter, plotted against IDR. For high dose-rates ( $>5.2 \mathrm{cGy} / \mathrm{h}$ ) the correction is taken constant ( $9 \%$ ), while for the lower dose-rates ( $<5.2 \mathrm{cGy} / \mathrm{h})$, the attenuation caused by the catheter was approximated by a logarithmic correction ( $Y=$ $13.835 \ln ($ IDR $)+33.1)$.

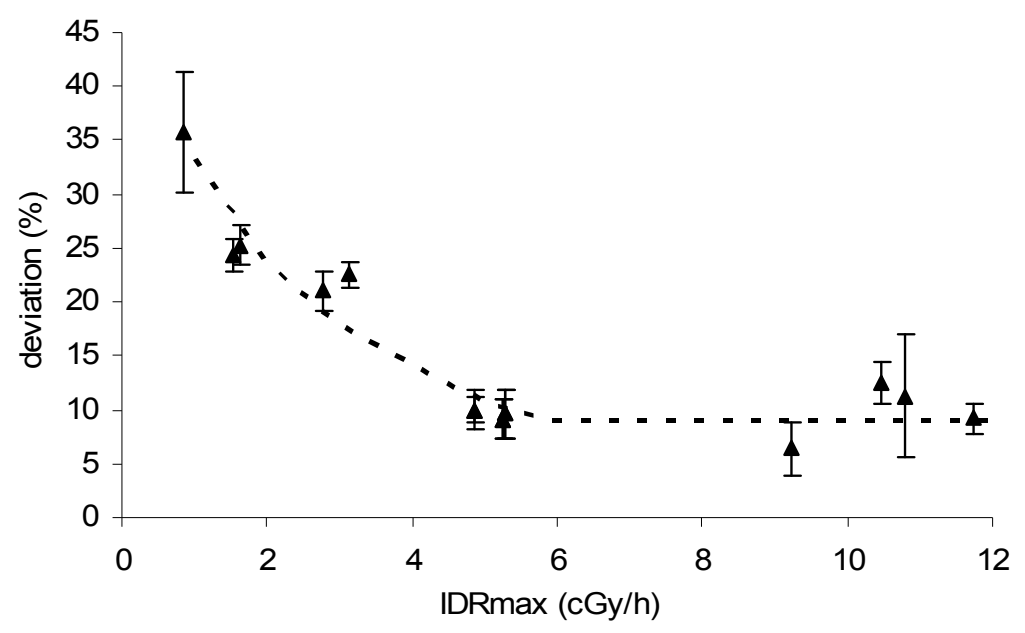

Figure 3: Catheter attenuation, as a function of the initial dose rate.

\subsubsection{Temperature dependence of the MOSFET-array response}

The influence of temperature on the sensitivity of the detector is illustrated in figure 4 . Because the results are based on single measurements, a relatively large fluctuation in the data was observed. However, a linear fit through these data points demonstrated an evident deviation between MOSFET sensitivity for room temperature compared to patient temperature, showing that the MOSFET sensitivity increases with increasing temperature. The ratio of the measurements performed at room-temperature compared to body-temperature is on average 1.11 and the temperature coefficient is $0.6 \% /{ }^{\circ} \mathrm{C}$. This means that the dose values during patient measurements will be on average $11 \%$ higher compared to the values defined during phantom measurements. A correction is therefore required. When the MOSFET-temperature is constant, a fixed correction of $11 \%$ can be applied to the in vivo dose values. 


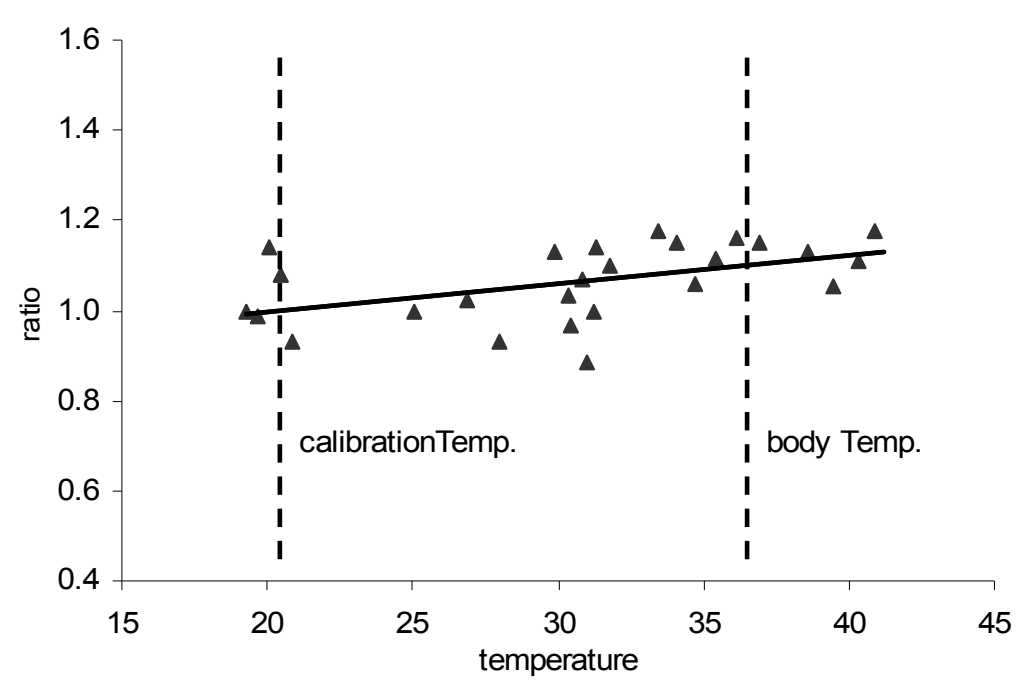

Figure 4: Temperature dependence of the MOSFET array for ${ }^{125} /$ photons. The dotted vertical lines indicate both calibration and patient temperature. The dose readings ( $\mathbf{\Delta})$ are related to room temperature.

\subsubsection{Total measurement uncertainty}

The total measurement accuracy, determined during the simulation of the clinical plan (at room-temperature), corrected for catheter attenuation, can be derived from figure 5 . The shapes of the measured and calculated curves correspond reasonably well. The observed average deviation between measured and calculated dose values is $4.9 \%$ $\pm 8.3 \%$ (1SD) for MOSFET-position 1 and. $-0.4 \% \pm 7.6 \%$ (1SD) for MOSFET-position 2. The overall mean of both measurements is $2.3 \% \pm 8.0 \%$ (1SD), the measured dose values being higher than the calculated dose values.

The uncertainty in the measurements is higher in the low-dose-rate regions compared to the high-dose-rate region, due to increase of the noise/signal ratio of the dosimeter. For dose-rates below $4.8 \mathrm{cGy} / \mathrm{h}$, the measurement uncertainty is $9.5 \%$ (1SD). For dose-rates of $4.8 \mathrm{cGy} / \mathrm{h}$ and higher, the uncertainty is $4.8 \%$.

\subsubsection{Patient measurements}

The position of the MOSFET-array, relative to the prostate $200 \mathrm{~Gy}$ isodose line in a patient, is presented in figures $6 a$ and $6 \mathrm{~b}$, showing a sagittal view and an oblique view, respectively. Figure $b$ illustrates that the $200 \mathrm{~Gy}$ isodose line and MOSFET-position do not overlap, demonstrating that the dose in the urethra does not exceed $200 \mathrm{~Gy}$. 


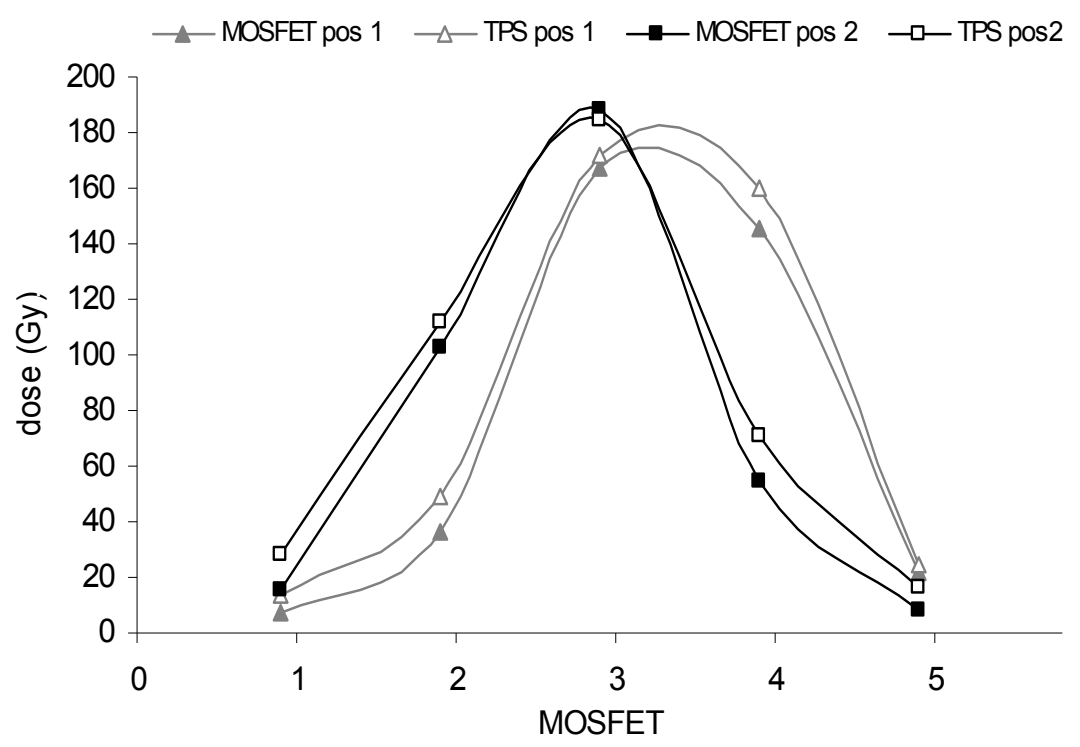

Figure 5: Measured MOSFET dose values for position 1 (pos 1) and position 2 (pos 2), corrected for catheter attenuation and dose values calculated with the treatment planning system (TPS), plotted against the initial dose rate. Pos 1 and pos 2 are shifted $1 \mathrm{~cm}$ relative to each other.

The measurements demonstrated that the mean IDR in the bladder neck, observed by MOSFET 1, was $0.7 \mathrm{cGy} / \mathrm{h} \pm 0.3 \mathrm{cGy} / \mathrm{h}$ (1SD). With distance from the bladder towards the prostate gland the dose-rates increased and reached their maximum inside the prostatic urethra where a mean IDR of $5.9 \mathrm{cGy} / \mathrm{h} \pm 1.3 \mathrm{cGy} / \mathrm{h}$ was observed. The highest dose-rates were observed for MOSFETs 3 and 4, depending on the length of the prostate. Extrapolated to the accumulated dose (D), the maximum observed dose was $197 \mathrm{~Gy}$. Towards the perineum, dose-rates decreased to $1.6 \mathrm{cGy} / \mathrm{h}$, as measured by MOSFET 5.

The mean deviation between measured and calculated dose values corrected for temperature dependence of the detector response and catheter absorption is $9.2 \%$ $\pm 22.3 \%$ (1 SD). This rather large deviation is mainly due to uncertainties in the lower dose area (extrapolated dose values $<100$ Gy or IDR $<4.8 \mathrm{cGy} / \mathrm{h}$ ), where a mean deviation of $12.0 \% \pm 24.7 \%$ (1 SD) was observed. In the high-dose area (accumulated dose $>100 \mathrm{~Gy}$ ), in or close to the prostatic gland, the mean deviation was $1.7 \% \pm 10.7$ $\%$ (1 SD). The global dose deviation, given as the dose relative to the highest dose value measured with a MOSFET in a particular patient, is however a more realistic way of expressing the deviations in this low dose region. In this way, the mean deviation of all points was $1.6 \% \pm 9.2 \%$ ( $1 \mathrm{SD}$ ) and for the lower dose region (<100 Gy) $1.5 \%$ 
$\pm 7.2 \%$ (1SD). Both corrected and uncorrected global dose deviations, relative to the maximum dose value, are presented in figure 7 .
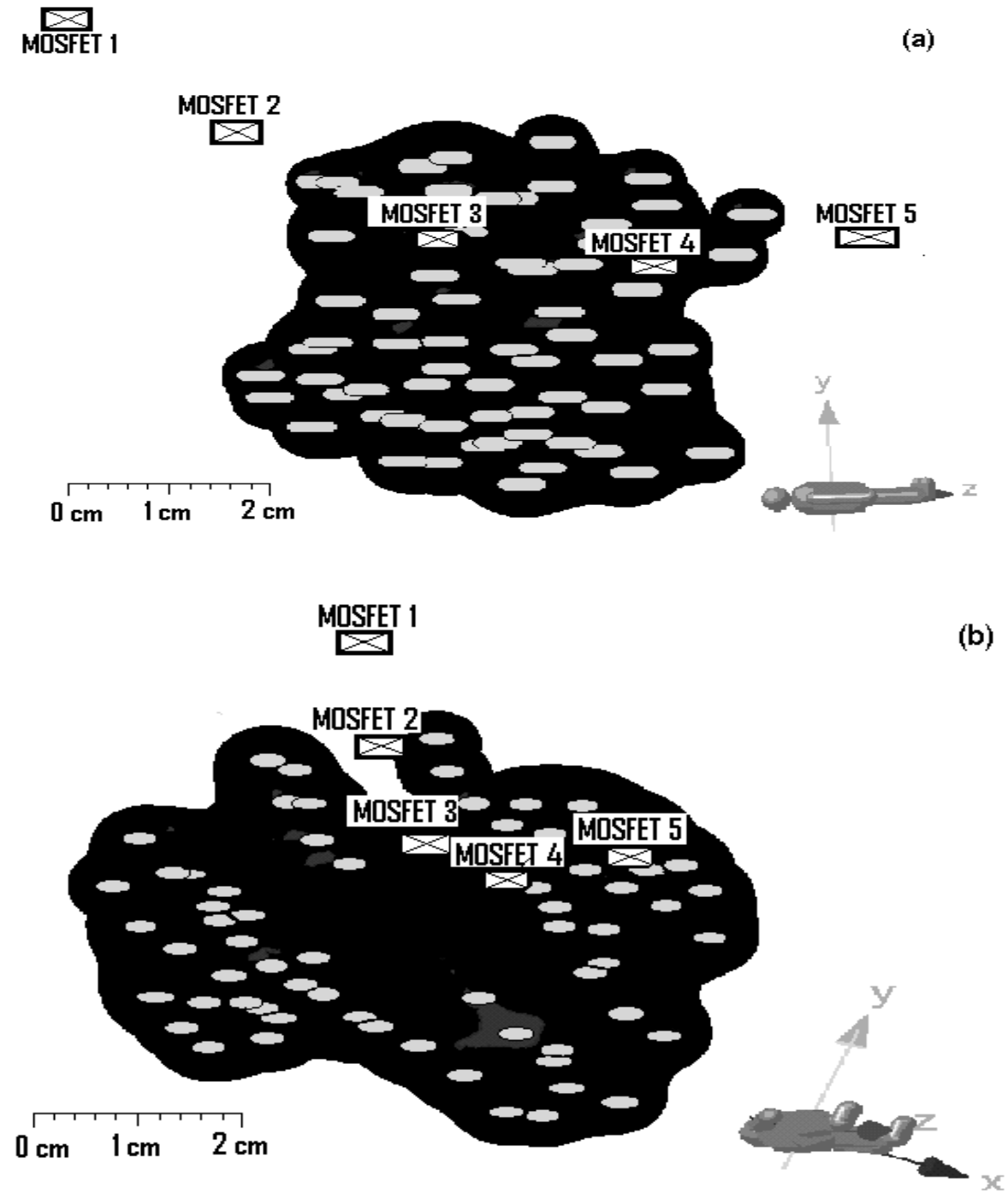

Figure 6: Three-dimensional illustration of the MOSFET array position relative to the seeds and

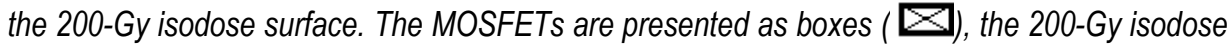
surface is presented as a black cloud. A lateral view (a) and an oblique-transversal view (b) are presented. 


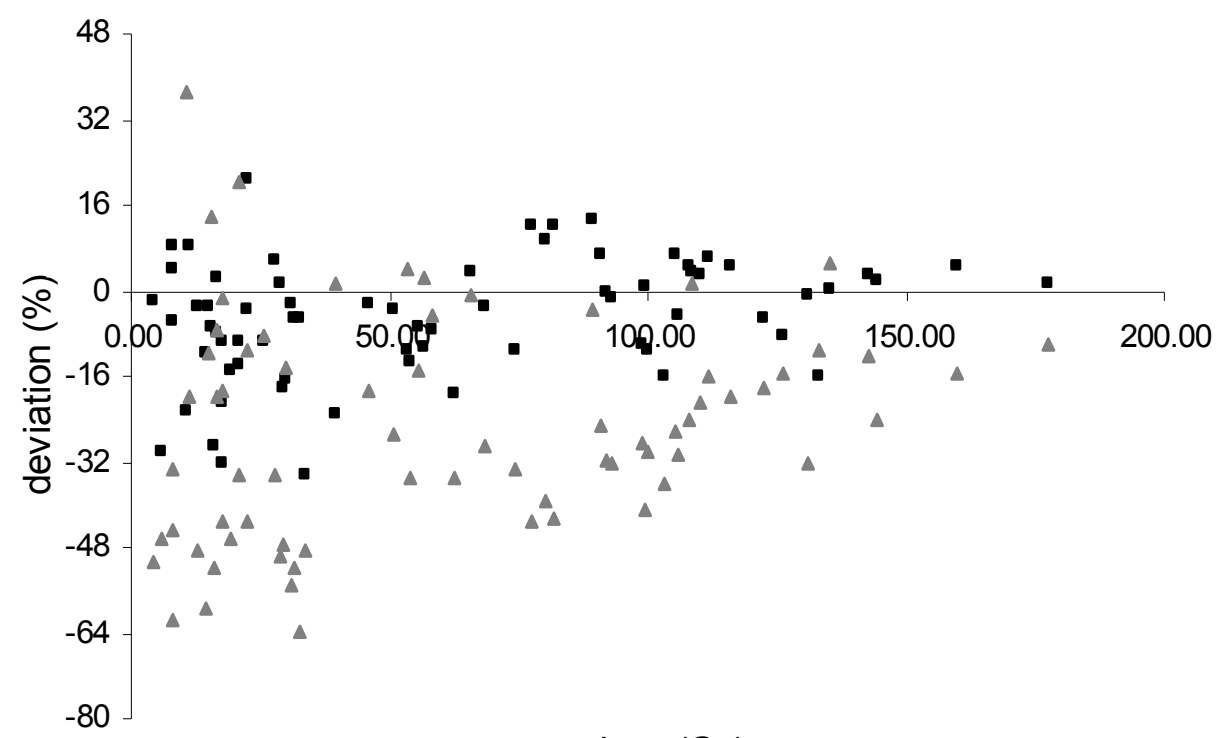

dose (Gy)

Figure 7: Uncorrected results of all patient measurements relative to calculated extrapolated dose values ( $\mathbf{\Lambda})$, and the same extrapolated results corrected for temperature dependence of the MOSFET array response and catheter attenuation, presented as the global dose deviation $(\mathbf{\square})$, that is, deviations in percentages of the maximum dose measured in each experiment.

\subsection{DISCUSSION}

In this study we demonstrate that, in order to perform in vivo dosimetry in brachytherapy, a good validation procedure of the detector system under clinical conditions is very important. If detectors used for this purpose have well-known characteristics, in vivo dosimetry is a good tool for QA of the treatment process and a prerequisite to relate dosimetric parameters to clinical outcome. In addition to the use of in vivo dosimetry after TIPPB, this approach could also be used for other brachytherapy techniques, such as HDR brachytherapy of prostate cancer or of other cancer sites.

The linear MOSFET-array, is suitable for measurements after TIPPB. In this study a high-sensitivity array was inserted into the urinary catheter. When fully inserted into the catheter, generally one MOSFET is located in the bladder neck, 2 or 3 MOSFETs are located in the prostate and 1 or 2 MOSFETs are located beneath the prostate. A mean global deviation (related to the maximum dose values) of $1.6 \% \pm 9.2 \%$ (1 SD) between in vivo and calculated results was found during our in vivo measurements. This result is within the measurement uncertainty and is very promising. 
During patient measurements the MOSFET-array is located inside a catheter and the catheter material consequently reduces the measured dose values. In order to quantitatively compare the calculated and measured dose values, the latter ones have to be corrected for the catheter attenuation. The extent of the absorption depends on the angle of incidence of the radiation through the catheter material. We used an approach where we linked the distance between the MOSFET and the seeds, and thus the angle of incidence, to the IDR. For the higher dose-rate areas, in or close to the prostatic gland, a correction of about $9 \%$ is applied, while for the lower dose-rate areas the correction is a logarithmic function of IDR. This correction is not related to the MOSFET response, which is dose rate independent.

In this study a temperature dependence of the MOSFET-array response of about $11 \%$ was observed, when raising the temperature from $20^{\circ}$ to $37^{\circ} \mathrm{C}$. Ramaseshan et al..$^{13}$ described the temperature response of the micro-MOSFET, demonstrating a $0.5 \%$ variation when increasing the temperature from $20^{\circ}$ to $40{ }^{\circ} \mathrm{C}$. These different observations are due to fundamental design differences between the linear-array and the micro-MOSFET-dosimeter. In the MOSFET-array, the 5 MOSFET-dosimeters share a distant common MOSFET, located at the array's connector and always at room temperature, leading to less compensation of the threshold voltage variations of the 5 MOSFETs when they reach body-temperature. In the case of the micro-MOSFET, the dual-MOSFET-dosimeter design is implemented on a similar silicon-chip, with both sensors following similar temperature variations, leading to their differential response independent of temperature ${ }^{14}$. After the MOSFET-array's insertion inside the catheter, it is advised to allow a short waiting period ( $\sim 5$ minutes), in order to stabilize the MOSFET to body temperature before starting the measurements.

An extended set of phantom measurements was performed, to validate the detectorsystem under clinical conditions. For these measurements, seeds with an activity of approximately $0.6 \mathrm{U}(0.472 \mathrm{mCi})$ were used. The low dose-rates emitted by the seeds resulted in relatively long measurement times and higher noise/signal ratio. Using highactivity seeds for calibration and phantom measurements will reduce measurement times and may result in smaller measurement uncertainties ${ }^{5}$.

The total measurement uncertainty involved in this measurement method was determined by simulating the procedure in a phantom and comparing the in vivo dose measurement results with calculated dose values. The uncertainty of these measurements is $\pm 8.0 \%$ (1 SD). Based on these results, action levels for in vivo measurements of $\pm 16 \%$ (2 SD) should be achievable. When the dose difference exceeds the action level, the cause of the deviation between the planned and measured dose distribution will be investigated and that knowledge may be used for future implantations.

Cygler et al. ${ }^{5}$ described the use of MOSFET-detectors for dosimetric verification after TIPPB. In that study the feasibility of micro-MOSFET-detectors for QA and in vivo 
dosimetry of the urethra was investigated, showing that MOSFET-detectors are suitable for clinical dosimetry after TIPPB. In their study the micro-MOSFET was used and moved along the urethra in $1 \mathrm{~cm}$ steps, because at the time of that study, an array of MOSFETs was not yet available. This method is however time consuming and introduces an uncertainty in MOSFET-position. Clinical studies describing the use of a linear-MOSFET-array are limited ${ }^{9,15,16}$. The results on angular response agree with the results in this study, however data about catheter absorption and temperature response were not provided.

Brachytherapy is a good tool for the treatment of prostate cancer. Although this is an effective treatment option, significant complications involving the genitourinary tract may result. A consequence of exceeding the maximum tolerable urethra dose level is acute urinary morbidity ${ }^{17}$. Other treatment complications involve radiation proctitis and the development of a rectal ulcer ${ }^{18}$. Real-time dosimetry during the implantation procedure is a promising tool to check and adjust the treatment conditions and dose values in the genitourinary tract during the implantation. Real-time dosimetry gives the opportunity to make changes in needle positioning during the procedure and avoid unacceptable dose values in organs at risk due to inaccuracies in needle positioning or seed activity. Real-time dosimetry has not yet been described and is the subject of further study in our department.

\section{ACKNOWLEDGEMENTS}

We like to thank Raymond Pijls, BSc, head of our brachytherapy facility, for his help during the phantom and patient measurements, and Abdelbasset Hallil, $\mathrm{PhD}$, for his advice during the measurements. 


\section{REFERENCES}

1. Marcu L, Quach K. The role of post-implant dosimetry in the quality assessment of prostate implants. The RAH experience. Australas Phys Eng Sci Med 2006;29:310314.

2. Chibani $\mathrm{O}$, Williamson JF, Todor D. Dosimetric effects of seed anisotropy and interseed attenuation for 103Pd and 125I prostate implants. Med Phys 2005;32:25572566.

3. Chibani O, Williamson JF. MCPI: a sub-minute Monte Carlo dose calculation engine for prostate implants. Med Phys 2005;32:3688-3698.

4. Carrier JF, D'Amours M, Verhaegen F, et al. Postimplant dosimetry using a Monte Carlo dose calculation engine: a new clinical standard. Int J Radiat Oncol Biol Phys 2007;68:1190-1198.

5. Cygler JE, Saoudi A, Perry G, et al. Feasibility study of using MOSFET detectors for in vivo dosimetry during permanent low-dose-rate prostate implants. Radiother Oncol 2006;80:296-301.

6. Nath R, Anderson LL, Luxton G, et al. Dosimetry of interstitial brachytherapy sources: recommendations of the AAPM Radiation Therapy Committee Task Group No. 43. American Association of Physicists in Medicine. Med Phys 1995;22:209-234.

7. Salembier $C$, Lavagnini $P$, Nickers $P$, et al. Tumour and target volumes in permanent prostate brachytherapy: a supplement to the ESTRO/EAU/EORTC recommendations on prostate brachytherapy. Radiother Oncol 2007;83:3-10.

8. Roshau JN, Hintenlang DE. Characterization of the angular response of an "isotropic" MOSFET dosimeter. Health Phys 2003;84:376-379.

9. Hallil A, Cygler J, Brown $M$, et al. Radiation Response of a New Linear MOSFET Array Dosimeter. Med Phys 2004;31:1912-1913.

10. Lavallee MC, Gingras L, Beaulieu L. Energy and integrated dose dependence of MOSFET dosimeter sensitivity for irradiation energies between $30 \mathrm{kV}$ and 60Co. Med Phys 2006;33:3683-3689.

11. Meigooni AS, Yoe-Sein MM, Al-Otoom AY, et al. Determination of the dosimetric characteristics of InterSource125 iodine brachytherapy source. Appl Radiat Isot 2002;56:589-599.

12. Luxton $\mathrm{G}$. Comparison of radiation dosimetry in water and in solid phantom materials for I-125 and Pd-103 brachytherapy sources: EGS4 Monte Carlo study. Med Phys 1994;21:631-641.

13. Ramaseshan R, Kohli KS, Zhang TJ, et al. Performance characteristics of a microMOSFET as an in vivo dosimeter in radiation therapy. Phys Med Biol 2004;49:4031-4048.

14. Soubra M, Cygler J, Mackay G. Evaluation of a dual bias dual metal oxide-silicon semiconductor field effect transistor detector as radiation dosimeter. Med Phys 1994;21:567-572.

15. Jurkovic I, Sadeghi A, Prestige B, et al. In vivo MOSFET detector used in the postoperative dose assessment of I-125 prostate implants. Brachytherapy 2006;5:97. 
16. Cygler JE, Sadeghi A, Perry G, Hallil A, et al. Measurement of urethral dose profiles in prostate brachytherapy using a Linear-MOSFET-Array-Dosimeter. Radiother Oncol 2004;71(Suppl.2):92-93.

17. Locke J, Ellis W, Wallner $\mathrm{K}$, et al. Risk factors for acute urinary retention requiring temporary intermittent catheterization after prostate brachytherapy: a prospective study. Int J Radiat Oncol Biol Phys 2002;52:712-719.

18. Celebrezze JP, Jr., Medich DS. Rectal ulceration as a result of prostatic brachytherapy: a new clinical problem: report of three cases. Dis Colon Rectum 2003;46:1277-1279. 


\title{
CHAPTER
}

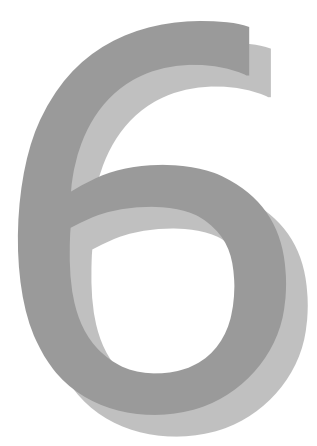

\section{Real-time dosimetry with a linear MOSFET array to evaluate the urethra dose during permanent implant brachytherapy using ${ }^{125 \mid}$}

Int. J. Radiation Oncology Biol. Phys., in press

\author{
Esther J. Bloemen-van Gurp \\ Björk K.C. Haanstra, \\ Lars H.P. Murrer \\ Francis C.J.M. van Gils \\ Andre L.A.J. Dekker \\ Ben J. Mijnheer \\ Philippe Lambin
}




\section{ABSTRACT}

Purpose: To develop a technique to monitor the dose-rate in the urethra during permanent implant brachytherapy using a linear MOSFET-array, with sufficient accuracy and without significantly extending the implantation time.

Methods and materials: Phantom measurements were performed to determine the optimal conditions for clinical measurements. In vivo measurements were performed in 5 patients during the ${ }^{125} \mathrm{l}$ brachytherapy implant procedure. To evaluate if the urethra dose obtained in the operation room with the ultrasound transducer in the rectum and the patient in treatment position is a reference for the total accumulated dose, additional measurements were performed after the implantation procedure, in the recovery room.

Results: In vivo measurements during and after the implantation procedure agree very well, illustrating that the ultrasound transducer in the rectum and patient positioning do not influence the measured dose in the urethra. In vivo dose values obtained during the implantation are therefore representative for the total accumulated dose in the urethra. In 5 patients the dose-rates during and after the implantation were below the maximum dose-rate of the urethra, using the planned seed distribution.

Conclusion: In vivo dosimetry during the implantation, using a MOSFET-array, is a feasible technique to evaluate the dose in the urethra during the implantation of ${ }^{125}$ | seeds for prostate brachytherapy. 


\subsection{INTRODUCTION}

Transperineal Interstitial Permanent Prostate Brachytherapy (TIPPB) using ${ }^{125}$ seeds has become a widely practiced treatment for early stage prostate cancer. The risks of normal tissue complication in this treatment depend on the dose received by the organs at risk (OAR), such as the urethra. The dose in the urethra is based on calculations of the treatment planning system (TPS) and a post-implant CT-scan. There are, however, various sources of uncertainty that may influence the actual dose in the urethra such as uncertainties in: seed activity, seed position, delineation of the urethra on the US image, and uncertainties in the dose calculation. Several studies described urethral strictures after a permanent prostate implant $t^{1,2}$.

In vivo dosimetry is a technique to assess the actual absorbed dose per unit time (dose-rate) in the urethra. In our previous study ${ }^{3}$, in vivo dose values were obtained after TIPPB for 17 patients. We demonstrated that a linear-MOSFET-array is suitable to evaluate the dose-rate in the urethra ${ }^{3}$. In vivo dosimetry during the implantation (i.e. real time), gives next to the in vivo dose data also the opportunity to constantly monitor the actually delivered dose-rate in the urethra during the implantation. As a result, in vivo dosimetry may assist in reducing the incidence of early or late urinary side effects.

The goal of this study is to develop a technique to monitor the dose-rate in the urethra during an implantation, with sufficient accuracy and without significantly extending the implantation time.

\subsection{MATERIALS AND METHODS}

In vivo dosimetry is a technique to monitor the dose-rate during the seed implantation. In this study, a detector was placed in the urethra during TIPPB, with the opportunity to measure the dose-rate in this OAR during the implantation. For the measurements we used extra high sensitive dual Metal Oxide Semiconductor Field Effect Transistors (MOSFETs), model TN1002-LA5 (MOSFET-array, Best Medical Canada, Ontario). The array contains five MOSFETs, placed on a flexible wire with $2 \mathrm{~cm}$ inter-MOSFET distance. It is narrow (1.5 mm wide, $1.3 \mathrm{~mm}$ thick, $460 \mathrm{~mm}$ long) and waterproof, which makes it suitable for measurements inside a patient. The calibration coefficient of the detectors, converting the radiation-induced voltage shift into dose, decreases with increasing energy in the $\mathrm{keV}$ range $\mathrm{e}^{4}$. Therefore, calibration was performed with the same radiation type as used for the clinical measurements. Other authors demonstrated drifts along the entire lifetime of the device for low x-ray energies ${ }^{4}$. To avoid underestimation of dose measurements, a recalibration of the device was performed periodically $(\sim 2000 \mathrm{mV})$. The Mobile MOSFET Dose verification system (TN-RD-70W, Best Medical Canada, Ontario), using Bluetooth to transfer the data 
wireless to a computer installed with the MobileMOSFET software, was used for doseread out. Because of the wireless readout, galvanic separation is ensured and it is safe to perform measurements in the operation room. Automatic dose readout was used and each dose measurement (cGy) was added to the stored sum of the previous measurements. Such a procedure has the advantage that relatively short integration times can be applied because dose values accumulate during the implantation, and more accurate dose information is obtained. Dose-rate was obtained by subtracting 2 sequentially obtained measurements, and the results were extrapolated to an 1 hour time period (cG/h).

For implantation we used a modified peripheral loading technique. Before implantation, a treatment plan (pre-plan) was made based on transrectal ultrasound images with the patient in treatment position (PSID 4.1 system, Sonotech, Bellingham, UK). Needles were loaded with single seeds or strands of seeds, based on the TPS data. The seeds were inserted into the prostate under ultrasound guidance. A template was used for needle positioning and the template coordinates were derived from the pre-plan data. During the implantation, the position of the seeds in the prostate was depicted on the longitudinal US images, and the dose-rate provided by the treatment plan was adapted subsequently. The final dose distribution after the implantation was defined as postplan. The calculations of the TPS were based on the TG-43 protocol of the American Association of Physicists in Medicine 5 .

A Foley-catheter, a silicone two-way balloon catheter with an outside diameter of $6 \mathrm{~mm}$ (18 French) and a length of $40 \mathrm{~cm}$ (Ref 170605, Teleflex Medical BV) was used to visualize the urethra. The MOSFET-array was positioned into the catheter. Correction for the influence of the catheter on the dose-rate was necessary for comparison of in vivo and calculated dose values, because the TPS does not take inhomogeneities into account. A correction of $9.1 \%$ was used for the MOSFETs in or close to the prostate, having dose-rates higher than $5.1 \mathrm{cGy} / \mathrm{h}$; for dose-rates lower than $5.1 \mathrm{cGy} / \mathrm{h}$ we used a dose-rate dependent correction as described in our previous study 3 . An 11\% correction for temperature dependence, as a result of changes in MOSFET sensitivity between room (calibration) temperature and body temperature, was used ${ }^{3}$. The uncertainties on the phantom and patient measurements are given as one standard deviation (1 SD) of the mean.

\subsubsection{Phantom measurements}

In vivo measurements to obtain the dose-rate during the implantation of the seed strands were performed in a phantom in order to: (1) determine the dependence of dose-rate on time for 3 clinically used implantation techniques and (2) to simulate the clinical procedure in a phantom. For these measurements, an in-house made phantom filled with gelatin was used. The phantom consisted of a PMMA casing $(10 \mathrm{~cm} \times 10 \mathrm{~cm}$ $x 10 \mathrm{~cm}$ ) with 49 holes drilled in its wall, comparable to the template used during the 
patient measurements. The phantom contained a urine-catheter for the placement of the array and was filled with of a solution of gelatin in water. Forty seeds with an activity of $0.488 \mathrm{mCi}(0.620 \mathrm{U})$ were used in strands of 2 or 3 seeds, mimicking the configuration of a clinical plan. The seeds were implanted through the wall of the phantom into the gelatin, using a seed distribution typical for a patient treatment. The time between the placements of the seeds in the gelatin was sixty seconds.

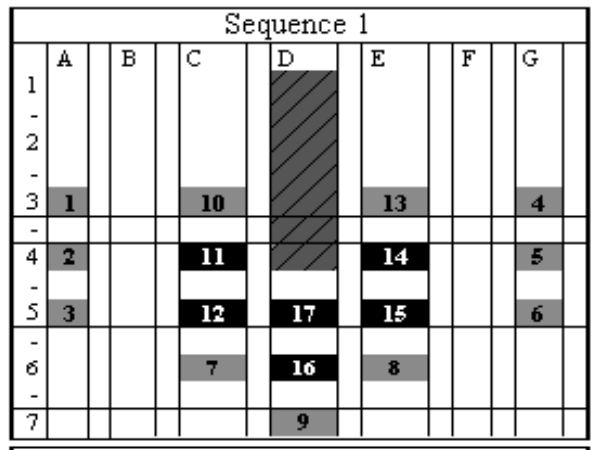

(a)

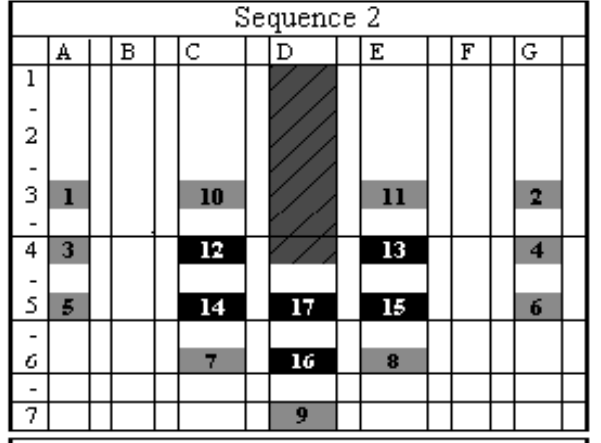

(b)

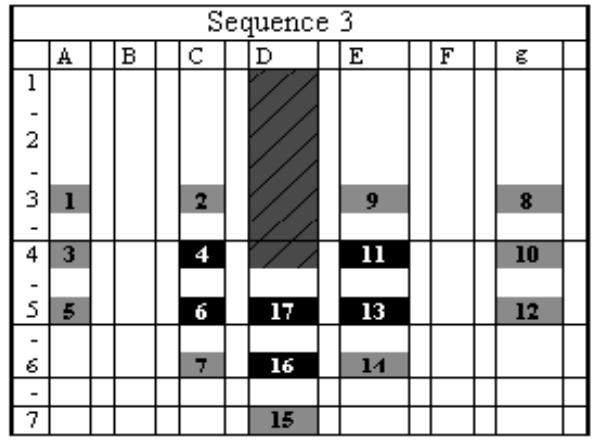

(C)

Figure 1: Template used for needle placement, positioning the seeds according to; a: sequence 1, in circles from the outside to the inside; b: sequence 2, zigzag from the outside to the inside and c: sequence 3, using the conventional sequence. The black boxes contain a strand of 3 seeds, the grey boxes a strand of 2 seeds and the shaded dark grey box illustrates the urinecatheter. 


\subsubsection{Phantom test to determine the dose-rate increase during implantation}

Measurements were performed during the implantation to determine the dependence of dose-rate on time. Besides our in-house used implantation sequence, two other sequences were tested with the aim to determine whether our in vivo measurement procedure can be extended to implantations performed by physicians using other implantation sequences. A constant time interval between the insertions of the strands and a fixed seed distribution was used. The integration time used to collect the dose was 30 seconds. The results were used; (1) to assess the most steep and critical part of the dose-rate for the urethra during the implantation and (2) to determine the increase in dose-rate between subsequent needles. Having a nearly linear dose-rate increase would facilitate the prediction of the dose-rate after the implantation of a new strand. Figure 1a shows sequence 1, where the seeds were implanted in circles into the gelatin from the outside to the inside. The numbers in the boxes illustrate the needle sequence. The design of sequence 2 (figure $1 \mathrm{~b}$ ) was based on a placement zigzag from the outside to the inside. Sequence 3 (figure 1c) illustrates the design of needle placement as used in our hospital, starting with the seeds in the left side of the prostate, continuing with the seeds in the contra-lateral side and finishing with the seeds under the urethra.

\subsubsection{Simulation of the clinical procedure in a phantom}

In the patient procedure, the final dose-rate at the end of the implantation should be restricted between a minimum dose-rate as obtained from the treatment plan and the maximum tolerable dose-rate of the urethra $(9.7 \mathrm{cGy} / \mathrm{h}$, or an integrated dose of 200 Gy $\left.{ }^{6}\right)$. To mimic this procedure, we performed a simulation of the clinical practice in a phantom. In clinical implants the number of seeds depends on the size of the prostate, and generally more than 50 seeds are use in our institution. Compared to an average prostate implantation procedure, we used in this experiment less seeds. As a consequence the dose-rate in the catheter was not as high as during a patient treatment and the maximum tolerable dose level of the urethra was not a relevant parameter. As a result, we used the highest expected dose-rate in the urethra, determined from the TPS, as an indicator for the dose-rate limit during the implantation. In addition to the maximum acceptable dose-rate, also a minimum dose-rate value was used as an indicator to detect errors that result in an underdose. This value was determined as the dose-rate at a distance of $1 \mathrm{~cm}$ from the maximum dose-rate in the urethra. A distance of $1 \mathrm{~cm}$ was used because the MOSFETs were hardly visible in the US-scan and the MOSFET location does not necessarily coincide with the highest dose point in the prostate. The inter-MOSFET distance is $2 \mathrm{~cm}$ and the maximum distance from this highest dose point and the closest MOSFET detector is therefore 1 $\mathrm{cm}$ or less. Consequently at least one of the MOSFETs should have a reading higher 
than the minimal value, thus providing a general dose level check. Based on the measurements to determine the dose-rate increase during the implantation, the doserate is most critical in the last part of the implantation procedure. As a consequence, during this part the actual dose-rate was checked more accurately. Before implanting the last 2 needles, a short waiting period was introduced in order to collect 3 measurements. The results of these 3 measurements were averaged to obtain the dose-rate with a better accuracy and this average was compared to the maximum expected dose-rate. When the dose-rate was close to the maximum tolerable doserate, we used a simple approach, based on a nearly linear dose-rate increase (see Results) to determine the dose-rate increment after the implantation of the next strand. We used the actual dose-rate $(\dot{D} a c t)$ and the dose-rate before the implantation of the last strand $(\dot{D}$ last $)$ to determine the dose-rate after the placement of the next strand $(\Delta \dot{D})$ as: $\Delta \dot{D} \cong \dot{D} a c t+(\dot{D} a c t-\dot{D}$ last $)$. After implanting all seeds, again 3 measurements were performed before removing the array from the catheter, in order to obtain the final dose-rate more accurately.

\subsubsection{Patient measurements}

In vivo measurements were performed during the implantation of 5 patients. Before implantation the array was fixed with a rubber wedge into the urine catheter with the tip of the array touching the tip of the catheter. Using this setup results in a positioning of MOSFET 1 into the bladder base, MOSFETs 2, 3, and 4 in or close to the prostate and MOSFET 5 in the urethra apical of the prostate. The maximum allowable dose-rate was set to $9.7 \mathrm{cGy} / \mathrm{h}$, which results in an integrated dose of $200 \mathrm{~Gy}$, being the generally accepted maximum tolerable dose of this organ at risk ${ }^{6}$. The minimum doserate for the MOSFET in the high dose area was determined using the pre-plan, and the procedure as explained for the simulation measurement, taking the dose value at a distance of $1 \mathrm{~cm}$ from this highest dose-rate in the urethra. The integration time was 1 minute. The dose measurements were started when the physician started the implantation. The seed strands were implanted, starting with the seeds in the left side of the prostate, continuing with the seeds in the contra-lateral side and finishing with the seeds under the urethra, conform sequence 3 (fig 1). During the first part of the implantation, the dose-rate was only registered. For the last part of the implantation, where the dose-rate relative to the urethra tolerance is more critical, we averaged 3 measurements after the implantation of the last 3 strands and the needle retraction, to obtain more accurate results. The dose measurement was compared with the maximum tolerable dose-rate of the urethra. If the dose-rate was below the maximum dose-rate, and adding a new strand would not result in exceeding this maximum level, the next strand of seeds could be safely placed. If adding seeds would result in exceeding the maximum dose-rate, the needle placement could be reconsidered. Urethral dose values and prostate dose coverage are, however, closely linked 
together, and the influence of omitting or repositioning a strand to prevent too high dose values in the urethra, should always be related to prostate coverage. After the placement of the last strand, the dose-rate of the MOSFET with the highest reading was expected to be above the minimal dose level and below the maximum dose level. After implanting all seeds, again 3 measurements were collected before removing the array from the catheter, to obtain the final dose-rate after the implantation. The extra time associated with this procedure was about 6 to 8 minutes.

To determine the effect of geometric changes of the prostate, caused by the presence of the transrectal ultrasound probe and patient motion, additional measurements were performed in the recovery room directly after the implantation $(\dot{D} r e c)$. Drec values were compared with the final dose-rate results obtained in the operation room $(\dot{D} r t)$.

\subsection{RESULTS}

\subsubsection{Phantom measurements}

\subsubsection{Test to determine the dose-rate increase during the implantation}

The sequence simulation of needle placement demonstrated that all tested sequences resulted in a nearly linear dose-rate increase at the end of the implantation. The results, presented as the dose increase as a function of needle placement, are given in figure 2.

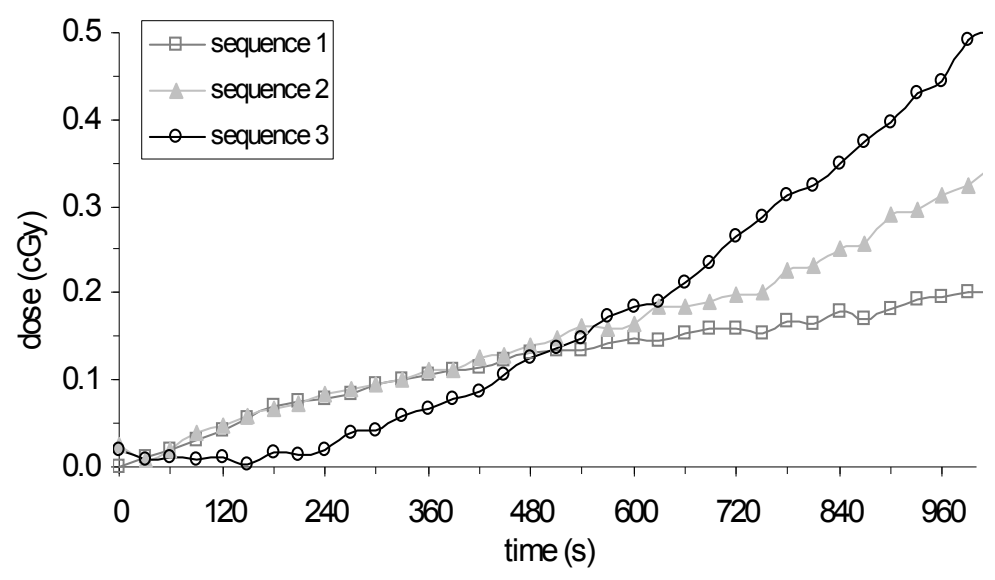

Figure 2: Illustration of the dose-rate increase during the implantation of the strands in the phantom for 3 implantation sequences. The symbols on the curve present the dose measurements. The time between the placements of the needles was $60 \mathrm{sec}$, which is indicated by the tick marks on the $x$-axis. The results of MOSFET 3 are presented. 


\subsubsection{Simulation of the clinical procedure in a phantom}

The result of the overall in vivo simulation measurement is shown in figure 3 . Point $A$ is the moment where needle number 17 ( 2 needles left) was retracted. After this point the average dose-rate ( 3 measurements, integration time 1 minute) over interval $[A, B]$ was (3.7 \pm 0.2$) \mathrm{cGy} / \mathrm{h}$ for MOSFET 3, the MOSFET in the highest dose region. The next needle $(\# 18)$ was placed between time points $B$ and $C$ resulting in an average doserate over interval $[C, D]$ of $(4.6 \pm 0.2) \mathrm{cGy} / \mathrm{h}$ for MOSFET 3. The last needle (\#19) was placed between time points $D$ and $E$, resulting in an average dose-rate over interval $[E$, F] of $(5.4 \pm 0.4) \mathrm{cGy} / \mathrm{h}$. The light grey line illustrates the minimum dose-rate that should be reached by the MOSFET in the high dose region, in this case by MOSFET 3 (3.6 $c G y / h)$. The dark grey line illustrates the highest expected dose-rate in the urethra, determined from the TPS and used as an indicator for the dose-rate limit, because the maximum tolerable dose level of the urethra was not relevant due to the limited number of seeds used for this measurement. Both MOSFET 3 and 4 were within the minimum and maximum dose-rate limits.

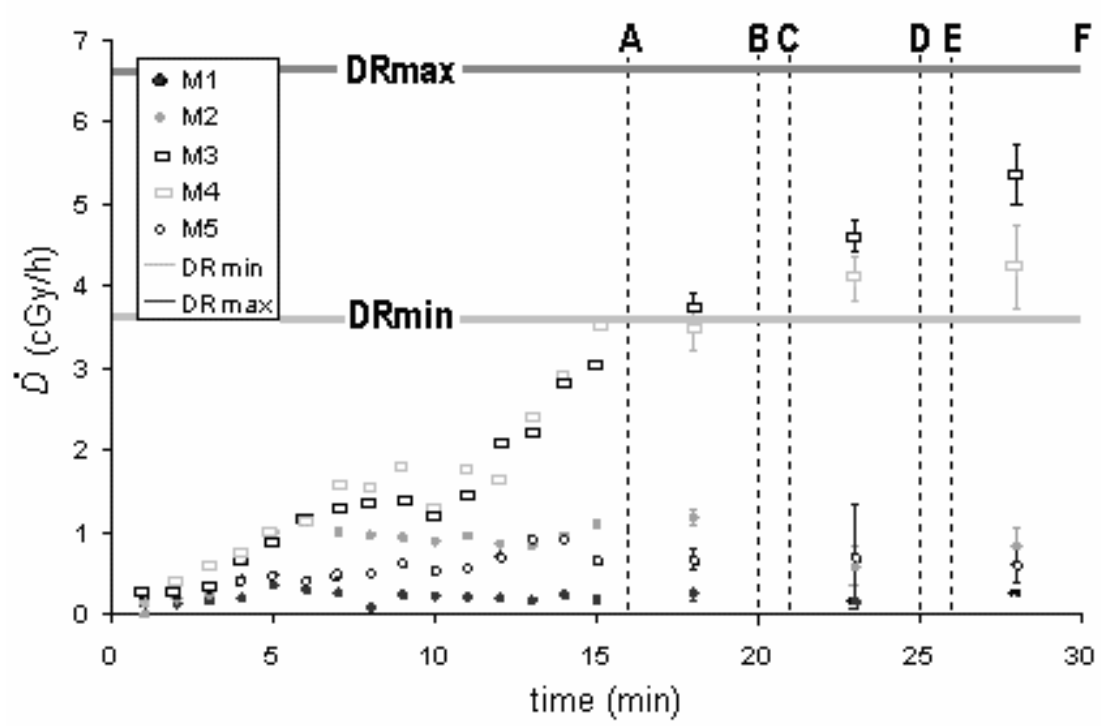

Figure 3: In vivo simulation measurement in a phantom using 5 MOSFETs (M1-M5). Points A, $C$ and $E$ are the moments where the last 3 strands were placed. The in vivo dose-rates, between interval $[A, B],[C, D]$ and $[E, F]$, were averaged and displayed with standard deviations.

\subsubsection{Patient measurements}

The final in vivo dose-rates for the 5 patients as obtained during the implantation are illustrated in figure 4a. A large standard deviation was observed due to the dose differences in the plans for the 5 patients and the differences in MOSFET position in 
the prostate between the patients. For all patients the in vivo dose-rate was below the maximum tolerable dose-rate of $9.7 \mathrm{cGy} / \mathrm{h}$.

To evaluate the small dose values obtained by MOSFET 1 and MOSFET 5 , the MOSFETs positioned at further distance from the prostate, we presented deviations between $\dot{D} r t$ and $\dot{D} r e c$ as a percentage of the maximum dose value. The deviations, calculated as: $(\dot{D} r e c-\dot{D} r t) / \dot{D} r t$, are presented in figure $4 \mathrm{~b}$. The results agree reasonably well and are within the standard deviation of the measurements.
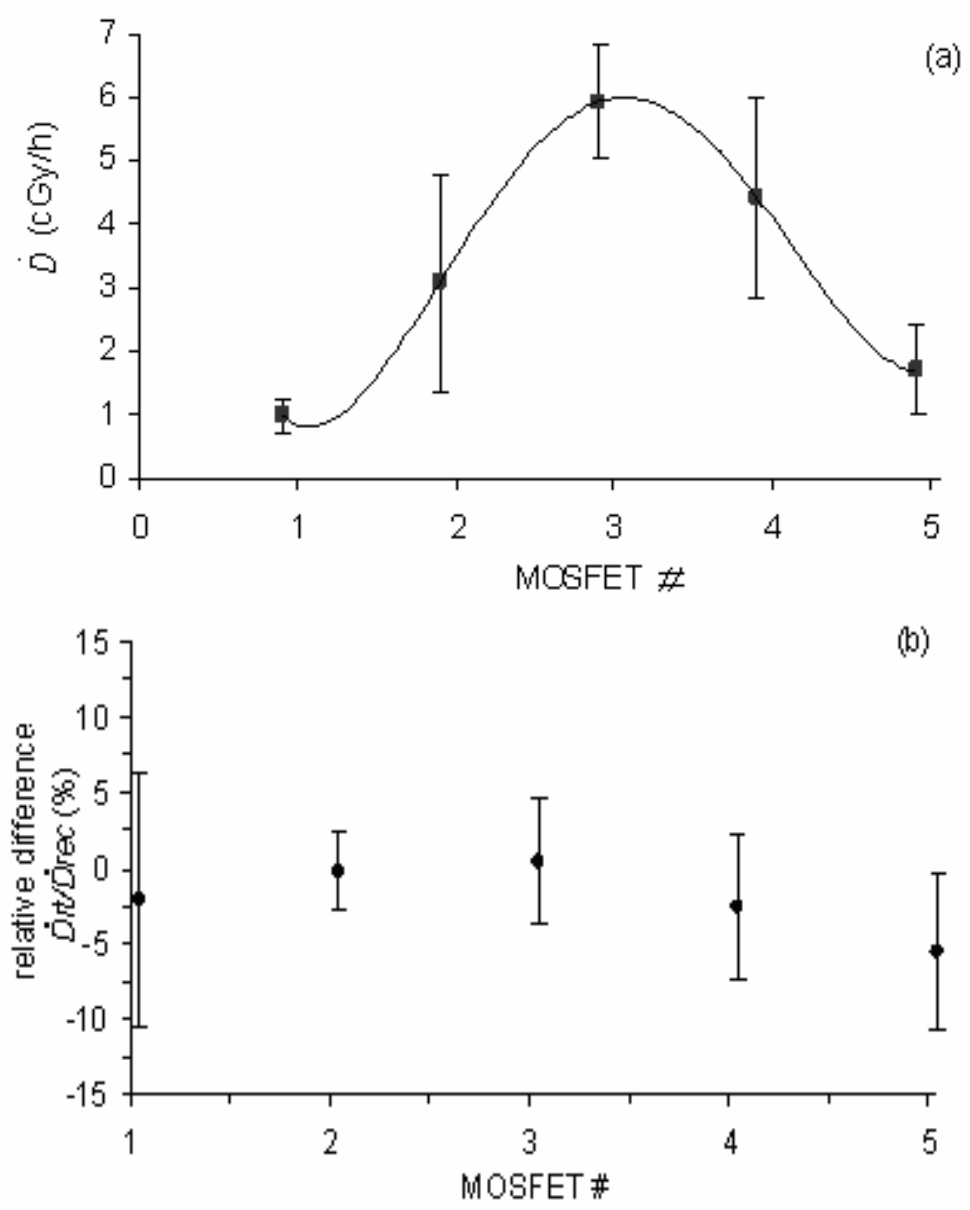

Figure 4: Patient measurements; a: final in vivo dose-rates for the 5 patients plotted against MOSFET position and connected by a line to illustrate the dose distribution over the urethra and b: deviations between $\dot{D} r t$ and $\dot{D} r e c$ for the 5 patients plotted as a percentage of the maximum dose value for each MOSFET position. Mean results and standard deviations are given. 
The minimum dose value that should be measured in the high dose area, determined from the pre-plan and corrected for the decay of the seeds, ranged between $113 \mathrm{~Gy}$ and 186 Gy for the 5 patients with a mean value of $(159 \pm 29)$ Gy. For all patients, however, the dose to the MOSFET in the highest dose region did not reach this minimum dose level. When evaluating the post-plan, the treatment plan that illustrates the dose distribution based on the final seed distribution in the prostate, we observed a lower urethra dose compared to the pre-operative planned urethra dose. The minimum dose values determined using the post-plan ranged between 79 Gy and 155 Gy with a mean of $(108 \pm 30) \mathrm{Gy}$, as indicated in figure 5 .

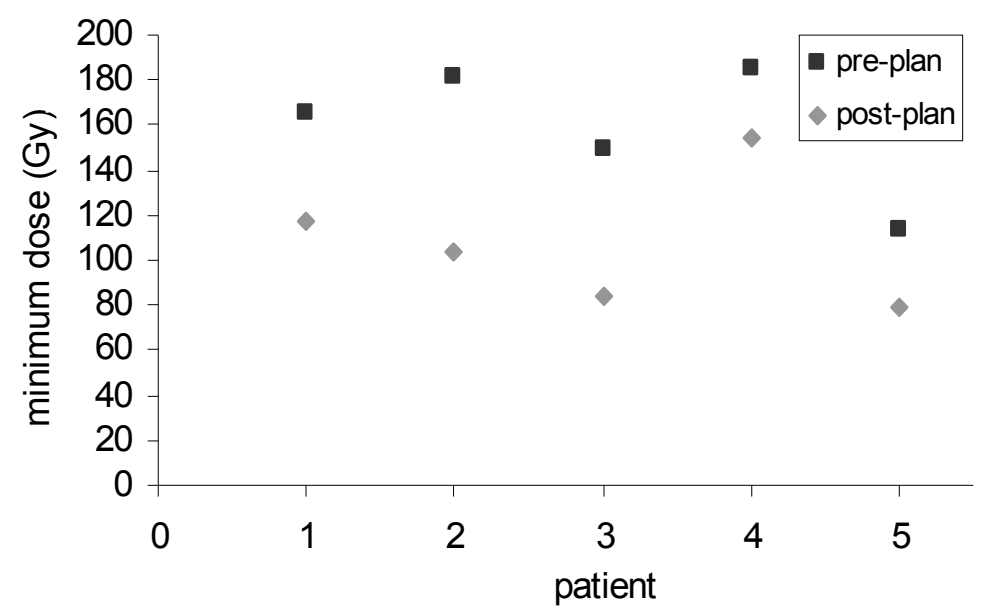

Figure 5: Minimum dose for the MOSFET in the high dose region, determined using the preplan and the post-plan for 5 patients. The black symbols (घ) are the minimum dose values determined using the pre-plan, while the grey symbols $(\diamond)$ are the minimum dose values determined using the post-plan.

The in vivo measurements of two patients are shown in figure $6 a$ and $b$. The dose-rate measured by MOSFET 3 over interval $[A, B]$, the moment when only 2 needles are left to insert, was $(5.4 \pm 0.1) \mathrm{cGy} / \mathrm{h}$ for plan $6 \mathrm{a}$ and $(4.4 \pm 0.1) \mathrm{cGy} / \mathrm{h}$ for plan $6 \mathrm{~b}$, obtained by averaging 3 measurements between interval $A$ and $B$. Between interval $B$ and $C$ the next needle was inserted, which resulted in a mean dose-rate over interval [C, D] of (5.3 $\pm 0.4) \mathrm{cGy} / \mathrm{h}$ and $(5.2 \pm 0.2) \mathrm{cGy} / \mathrm{h}$ for plan $6 \mathrm{a}$ and $6 \mathrm{~b}$, respectively. The last strand was implanted (interval $[D, E]$ ) and the final dose-rate after the implantation (interval $[E, F]$ ) was $(6.6 \pm 0.4) \mathrm{cGy} / \mathrm{h}$ for the first implant and $(5.8 \pm 0.3) \mathrm{cGy} / \mathrm{h}$ for the second implant. In figure $6 a$, both MOSFET 3 and MOSFET 4 are in the high dose-rate area, MOSFET 3 exceeded the minimum dose-rate value. In figure $6 b$, MOSFET 3 is in the high doserate area but the minimum dose-rate was not achieved. 


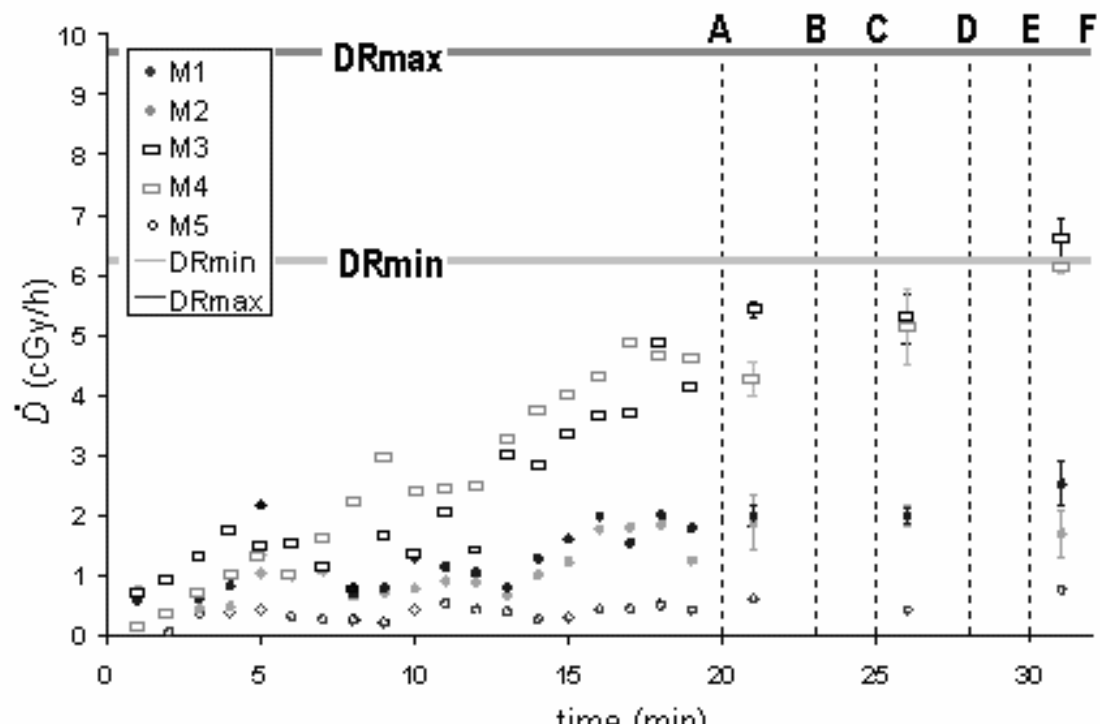

(a)

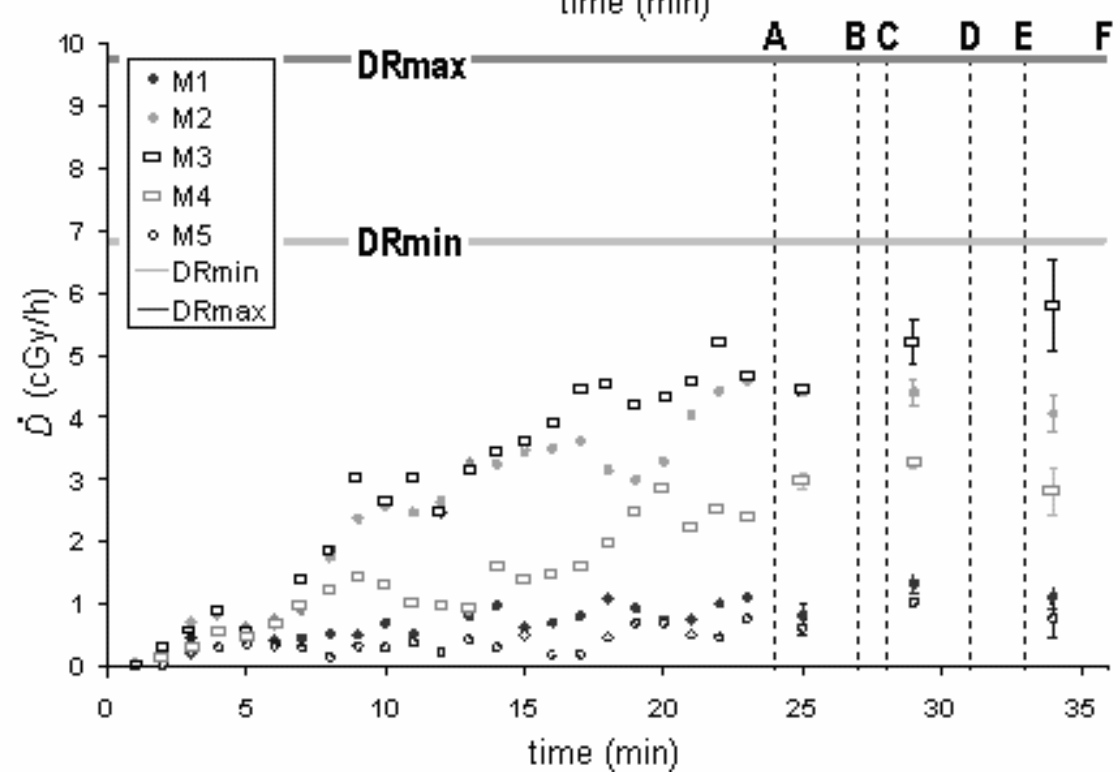

(b)

Figure 6: Results of in vivo dose-rate measurements of 2 patients (a and b). The light grey line is the minimum dose-rate value. The dark grey line is the maximum tolerable dose-rate limit for the urethra of $9.7 \mathrm{cGy} / \mathrm{h}$, or an integral dose of $200 \mathrm{~Gy}$. Point $A$ describes the moment when only 2 needles are left to insert. The time interval $[B, C]$ and $[C, D]$ comprises the last 2 strand implants. For interval $[A, B],[C, D]$ and $[E, F]$ an average dose-rate was determined. 


\subsection{DISCUSSION}

The method presented in this paper offers a clinical tool to relate actual delivered dose values to clinical side effects. During TIPPB, the final dose-rate is based on calculations performed by the TPS. Inhomogeneities in the prostate and surrounding tissues and inter-seed attenuation are not taken into account by our TPS. Uncertainties in the treatment procedure, such as seed position and seed activity can affect the urethral dose values. In vivo dosimetry is an appropriate tool to measure the dose-rate in the urethra during the implantation. It offers the opportunity to evaluate needle positioning during the procedure and to avoid unacceptable dose values in organs at risk.

Besides ${ }^{125}$, other isotopes such as ${ }^{103 P d}$ and ${ }^{131} \mathrm{Cs}$ are used for the treatment of prostate cancer. Given their somewhat different energy spectrum, combined with the energy dependence of the response of the detector in the low energy range ${ }^{4}$, slight differences in calibration factors may occur. For that reason it is important that calibration of the MOSFET detector in this low energy range is performed using isotopes identical to those used in clinical practice. The correction for catheter absorption should also be tested, because it depends on the photon energy of the seeds. The difference in dose-rate between the seeds does not influence the dose measurements, because the response of a MOSFET detector is dose rate independent ${ }^{7}$. In addition to the use of in vivo dosimetry during TIPPB, this approach can also be used for other brachytherapy techniques, such as HDR brachytherapy of prostate cancer or other cancer sites ${ }^{7}$.

The linear-MOSFET-array is a suitable detector for in vivo measurements during TIPPB. The 5 MOSFETs on the array provide us with dose information over the region ranging from the perineum to the bladder base. One should, however, be aware that the inter-MOSFET spacing is $2 \mathrm{~cm}$ and that the method may be rather insensitive to small hot spots (e.g. single seed close to urethra) located between the detector points. A correction of the dose values for attenuation by the catheter was used for comparison of the in vivo and calculated dose values, because the TPS does not account for catheter attenuation ${ }^{3}$. Also a correction for temperature dependence of the linear-MOSFET-array response of $11 \%$ was used ${ }^{3}$. Needle attenuation was not taken into account. In the first part of the implantation, when the accuracy of the dose determination is not very high, this was not relevant. In the last part of the implantation, used to evaluate the dose-rate in relation to the maximum acceptable dose-rate of the urethra, we used the measurements after the retraction of the needle.

During the first part of the implantation very low dose-readings are obtained. The accumulated dose in the prostate at the end of the implantation was about 2 cGy. Given the MOSFET sensitivity of $37.2 \mathrm{mV} / \mathrm{cGy}$, this dose corresponds to a MOSFETreading of about $74 \mathrm{mV}$. The dose for MOSFETs at larger distance from the prostate 
was even smaller, but at least $0.4 \mathrm{cGy}$, corresponding to a MOSFET reading of about $15 \mathrm{mV}$. Because dose values are accumulated during the implantation, the dose readout in the last part of the implantation will be more accurate compared to the accuracy in the first part. Furthermore, the dose values measured by the MOSFETs in the high dose area, or close to the prostate, are more accurate compared to the dose values measured at further distances from the prostate.

The dose-rate in the urethra, as calculated in the pre-plan, was higher compared to the dose-rate calculated in the post-plan, possibly due to seed placement inaccuracies and edema that occurs during the implantation. As a consequence, the in vivo measurements demonstrated dose-rates that were lower compared to the predicted dose-rates, calculated using the pre-plan. Dose coverage, however, was ensured, as demonstrated by the post-plan at the end of the implantation. The correlation between the lower urethral dose values and the seed distribution in the prostate is an object of further study.

The dose-rate obtained in the operation room at the end of the implantation and in the recovery room agree reasonable well, illustrating that the US transducer in the rectum and patient positioning do not influence the dose in the urethra. Hence, in vivo doses obtained during the implantation are representative for the total accumulated dose in the urethra at these MOSFET positions.

Clinical studies describing the use of a linear-MOSFET-array for measurements in the urethra are limited $3,8-10$. The effect of displacement and deformation of the prostate on the planned dose in the urethra, caused by the presence of the transrectal ultrasound transducer and movement of the patient, has, to the best of our knowledge not been described. In this study we demonstrate that in vivo dose-rates in the urethra, obtained during the implantation, yield a good prediction of the total accumulated dose in the urethra. Two uncertainties still remain in the prediction of the accumulated dose from the in vivo dose-rate values obtained during implantation; the occurrence of edema during and after the implantation, and the migration of individual seeds. The influence of these phenomena on the total accumulated dose has been investigated by several other groups ${ }^{11-14}$.

\subsection{CONCLUSION}

In vivo dosimetry, using a MOSFET array, is a promising technique to evaluate the dose rate and the accumulated dose in the urethra during the implantation of ${ }^{125}$ seeds for prostate brachytherapy. It offers a clinical tool to ensure maximum tolerable doses are not reached in this organ at risk by allowing on the fly adaptations to the treatment plan. In this study, for all patients the in vivo urethra dose-rate was below the maximum tolerable dose-rate. 


\section{REFERENCE}

1. Bittner N, Merrick GS, Wallner KE, et al. The impact of acute urinary morbidity on late urinary function after permanent prostate brachytherapy. Brachytherapy 2007;6:258266.

2. Wust $P$, Postrach J, Kahmann $F$, et al. Postimplantation analysis enables improvement of dose-volume histograms and reduction of toxicity for permanent seed implantation. Int J Radiat Oncol Biol Phys 2008;71:28-35.

3. Bloemen-van Gurp EJ, Murrer LH, Haanstra BK, et al. In vivo dosimetry using a linear Mosfet-array dosimeter to determine the urethra dose in $125 \mathrm{I}$ permanent prostate implants. Int J Radiat Oncol Biol Phys 2009;73:314-321.

4. Lavallee MC, Gingras L, Beaulieu L. Energy and integrated dose dependence of MOSFET dosimeter sensitivity for irradiation energies between $30 \mathrm{kV}$ and $60 \mathrm{Co}$. Med Phys 2006;33:3683-3689.

5. Nath R, Anderson LL, Luxton G, et al. Dosimetry of interstitial brachytherapy sources: recommendations of the AAPM Radiation Therapy Committee Task Group No. 43. American Association of Physicists in Medicine. Med Phys 1995;22:209-234.

6. Salembier $C$, Lavagnini $P$, Nickers $P$, et al. Tumour and target volumes in permanent prostate brachytherapy: a supplement to the ESTRO/EAU/EORTC recommendations on prostate brachytherapy. Radiother Oncol 2007;83:3-10.

7. Qi ZY, Deng XW, Huang SM, et al. Verification of the plan dosimetry for high dose rate brachytherapy using metal-oxide-semiconductor field effect transistor detectors. Med Phys 2007;34:2007-2013.

8. Prestidge BR. JI, Sadeghi AG., Salinas L., Bice WS. Postoperative urethral dose assessment of I-125 prostate implants using in vivo MOSFET detectors. Brachytherapy 2007;6:90.

9. Jurkovic I SA, Prestige B, et al. In vivo MOSFET detector used in the post-operative dose assessment of I-125 prostate implants. Brachytherapy 2006;5:97.

10. Hallil A. Cygler J BM, et al. Radiation Response of a New Linear MOSFET Array Dosimeter. Med Phys 2004;31:1912-1913.

11. Tanaka O, Hayashi S, Matsuo M, et al. Effect of edema on postimplant dosimetry in prostate brachytherapy using CT/MRI fusion. Int $\mathrm{J}$ Radiat Oncol Biol Phys 2007;69:614-618.

12. Leclerc G, Lavallee MC, Roy R, et al. Prostatic edema in 125 I permanent prostate implants: dynamical dosimetry taking volume changes into account. Med Phys 2006;33:574-583.

13. Aoki M, Miki K, Takahashi H, et al. [Changes of edema associated with I-125 prostate brachytherapy]. Nippon Igaku Hoshasen Gakkai Zasshi 2005;65:411-418.

14. Fuller DB, Koziol JA, Feng AC. Prostate brachytherapy seed migration and dosimetry: analysis of stranded sources and other potential predictive factors. Brachytherapy 2004;3:10-19. 

Discussion 

CHAPTER

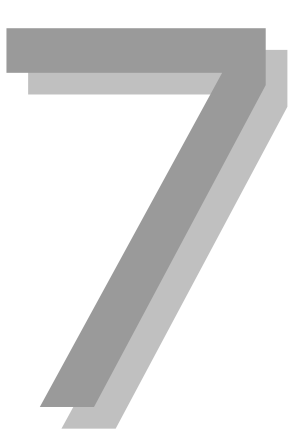

General Discussion and Future Perspectives 



\subsection{IN VIVO DOSIMETRY IN RADIOTHERAPY}

In vivo dosimetry plays an important role in a quality assurance programme in radiotherapy because its main goal is to protect patients from errors in treatment delivery. It can serve as an overall check of the treatment procedure to ensure that the prescribed and delivered dose agree within acceptable limits. It can also serve for dose verification during the implementation of a new treatment technique or as an indicator to assess the dose to organs at risk. Since January 2001, MAASTRO CLINIC uses MOSFET dosimeters as part of a QA programme of treatment delivery. In our institution we developed an in vivo dosimetry protocol, using MOSFETs, for all treatments with curative and elective intent. At the start of this project, the commercially available TN-RD-50 system (Thomson and Nielsen, a division of Best Medical Ottawa, Canada) patient verification system was used. For phantom measurements, to validate the detector, the MOSFET AutoSense system (TN-RD-60), developed for on-line radiotherapy applications, was provided for a brief period of time by Thomson and Nielsen and Pi-Medical, their Dutch representative, because the TN-RD-50 patient verification system does not allow on-line dose reading. In 2004/ 2005, our TN-RD-50 patient verification system was replaced by a mobile MOSFET system (TN-RD-70-W), a new wireless system that is entirely software driven. The mobile MOSFET system allows for remote control of one or more systems from a PC and dose readout without entering the room. At the moment this thesis was written, MAASTRO CLINIC has 7 linear accelerators and on each linac a mobile MOSFET dose verification system including the remote software is available. For brachytherapy purposes an additional mobile MOSFET dose verification system with an additional bias box, allowing for the use of 2 linear arrays simultaneously, is available.

In this thesis we demonstrated that an extensive validation procedure is essential to apply a specific type of detector and obtain accurate in vivo dose measurements. The calibration procedure and sensitivity variations of the detector system have to be determined for each measurement situation. In chapter 2, we investigated the accuracy of MOSFET detectors to measure dose to points in the region of a field junction in a split beam technique. The results demonstrated that in the junction region MOSFETs performed well but slightly overestimated the dose during measurements in the penumbra of the beam. A correction factor of $5 \%$ was used to correct the MOSFET results in this particular area. In chapters 3 and 4, the sensitivity variation of the detectors was determined in electron beams and in photon beams at extended distance, respectively. Instead of using correction factors for separate properties of the MOSFETs, an overall factor was used, converting the dose obtained under reference conditions to a dose value determined under clinical circumstances. Because these conversion factors were determined using an ionisation chamber as a reference instrument, sensitivity changes of each MOSFET are included in this factor. In chapter 3 , the MOSFET reading with the detector placed on the patient's skin without additional 
build-up, was converted to dose values at the depth of dose maximum. In chapter 4 , entrance and exit in vivo readings obtained without build up material and using the calibration factor determined under reference conditions were converted to midline dose data under TBI circumstances. In chapters 5 and 6, we used a linear-5-MOSFET array to investigate the sensitivity of the detector for measurements in the urethra. In this study, we demonstrated that a correction for temperature of $11 \%$ from room temperature to body temperature is necessary, due to the design of the array. The angular dependence of the detector is within $3 \%$ and is of the same magnitude as observed by other groups ${ }^{1,2}$

In external beam radiotherapy the use of in vivo dosimetry is getting more and more accepted. After validating the detector for a specific measurement situation, useful dose information can be obtained. To evaluate the in vivo dose values, measurement uncertainties for each measurement situation should be quantified. In this thesis, the measurement uncertainty of the detector and the uncertainties in the treatment process involved, without the influence of patient related factors, were determined for different treatment geometries. The mean MOSFET reproducibility of the detector was 2.0\% (1 SD) for readings at $100 \mathrm{mV}$ and $1.8 \%$ (1 SD) for readings at $200 \mathrm{mV}$, which resulted from 10 successive measurements, using the standard sensitivity of the bias supply. This is of the same order of magnitude as observed by other groups ${ }^{3,4}$. In electron beams we determined an overall uncertainty in dose determination of about 4\% (1SD), while the overall uncertainty in the dose determination using MOSFETs in our TBI setting was about $3.5 \%$ (1 SD).

In brachytherapy there has been a delay in the development of QA compared to external beam radiotherapy, where in vivo dosimetry is applied on a larger scale. Brachytherapy QA procedures are mainly focused on equipment or separate steps of the treatment process such as machine performance, accuracy of source positioning, implant reconstruction, radiation safety and consistency of the treatment planning procedure, mainly because accurate in vivo dosimetry is difficult to achieve. Compared to dose measurements in external beam radiotherapy, the dose uncertainty in brachytherapy is larger, due to the high dose gradients, the relatively low dose-rate and the complex photon energy spectrum of the sources. Our phantom measurements demonstrated an overall uncertainty of $8.0 \%$ (1SD) in this situation, which includes detector positioning and correction factor uncertainties. Cygler et al. ${ }^{1}$ used MOSFET detectors for in vivo dosimetry during permanent low dose-rate prostate implants and this group of investigators estimated the accuracy of the in vivo measurements in their study to be $\pm 3 \%$ (1 SD) at each spatial position. This result, however, was based on their calibration measurement reproducibility, using a higher seed activity compared with the clinical situation.

Before the implementation of in vivo dose measurements in routine daily practice, tolerance levels and action levels should be defined in order to evaluate the obtained dose value. A tolerance level, a variation from a standard or the permissible deviation 
from a specified value of a structural dimension, should be defined for each single step in the treatment procedure, resulting in an uncertainty of the final dose delivery within a pre-defined level. Action levels are, opposed to tolerance levels, decision tools applied by the radiation therapy technologists or other professionals who perform the in vivo dose measurements, and are linked to action. If the measurement result is within the action level, the measurement is accepted. If deviations exceed the action level, a specified action should be applied, because exceeding this threshold can be a result of inadvertent actions or accidental errors. Measurements that exceed the action level, in our institution, require a second measurement to exclude errors in the measurement procedure. In addition, depending on the amount of the deviation, all treatment data are checked and the medical physicist evaluates the measurement result. It is important that the action level is larger than the measurement uncertainty and the uncertainty in the final dose delivery, because too narrow action levels may result in unnecessary intervention, increased downtime of equipment and inefficient use of staff time. Optimal action levels, should be based on initial phantom and patient measurements. The action levels described in this thesis range from $7 \%$ in external beam in vivo dosimetry, up to $16 \%$ in brachytherapy dose verification. ESTRO-EQUAL recommends action levels of $5 \%$ for beam delivery in external beam radiotherapy using a mailed TLD system to check the dose delivered on axis in reference and nonreference conditions for external beam radiotherapy ${ }^{5}$. This level is, however, based on phantom measurements and does not include patient related factors. A radiotherapy department should make a compromise between accuracy and workload of a QA programme, which will depend on the tumor site and OARs involved. In addition to action levels for individual patient verification, a periodically evaluation of verification results of groups of patients will give the ability to detect systematic deviations. Due to the dose gradients and very low dose-rates involved, the action levels applied in external beam radiotherapy are generally not achievable for in vivo dose measurements in brachytherapy. Consequently in vivo dosimetry during brachytherapy does not provide high accurate dose information, but is nevertheless a good approach to detect gross errors.

MOSFET dosimeters are suitable detectors for a broad number of in vivo dosimetry applications such as dosimetry at extended distance, in the penumbra of a beam, for routine verification during photon and electron beam treatments and for measurements during brachytherapy in a catheter inside the patient. The detector mostly used in our study is a dual-bias dual-MOSFET detector. This detector consists of two identical MOSFETs, fabricated on the same silicon chip as presented in figure 1. In the linear array, the five MOSFET dosimeters share a distant common MOSFET, located at the array's connector, in order to reduce the size of the cross section of the array and make it suitable for measurements in a catheter. The two identical MOSFETs in both the single and the array dosimeter, operate at two different gate biases (1 and $15 \mathrm{~V})$. During irradiation each sensor will produce a threshold voltage shift, the gate voltage necessary to allow charge conduction through the MOSFET. The PC is on-line with the 
Reader Module and reading is obtained in real-time by passing current through the cable to the MOSFET chips. The difference between the two threshold voltage shifts is a linear function of absorbed dose ${ }^{6}$.

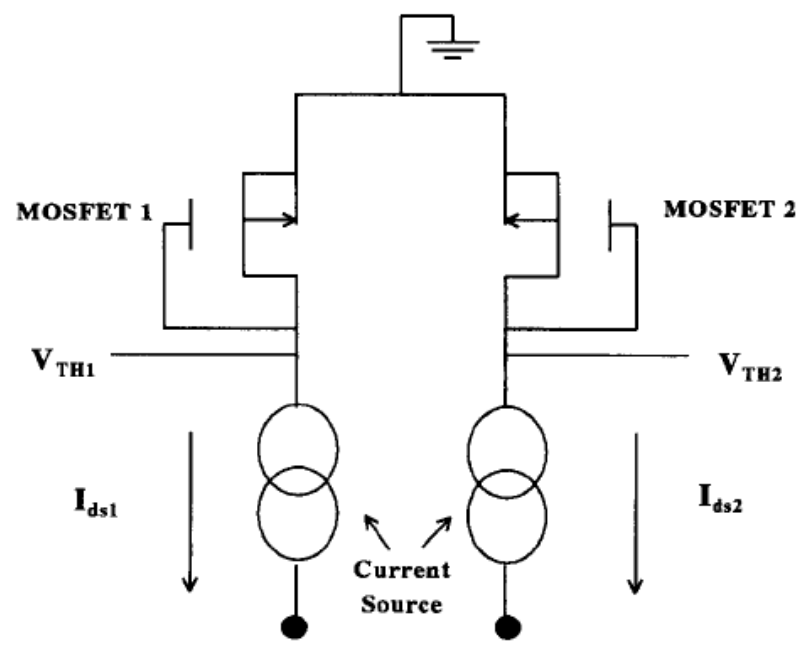

Figure 1: The basic structure of a dual-bias dual-MOSFET, having a readout circuit for the threshold voltages $V_{\mathrm{TH} 1}$ and $V_{\mathrm{TH} 2}$ of each MOSFET in the sensor pair and a current between the source and the drain ( $I_{d s 1}$ and $I_{d s 2}$ ) that is maintained during readout 6 .

In a dual-bias dual-MOSFET detector, both sensors follow similar temperature variations, leading to their differential response, which is almost independent of temperature ${ }^{6,7}$. The MOSFET array, however, where five MOSFET dosimeters are shared with one distant common MOSFET, located at the array's connector and therefore always at room temperature, has less compensation of the threshold voltage variations of the five MOSFETs when temperature increases. As a result, a temperature dependence of the MOSFET array response of approximately $11 \%$, when raising the temperature from 20 to $37^{\circ} \mathrm{C}$, was observed in our study.

MOSFET sensitivity increases as the incident energy decreases from $150 \mathrm{keV}$ to 10 $\mathrm{keV}$, the energy range in brachytherapy purposes ${ }^{8}$. This energy dependence is typical for most dosimeters and the effect is due to dose enhancement and increasing dominance of photoelectric absorption, while between $60 \mathrm{keV}$ and $90 \mathrm{keV}$ both photoelectric and Compton scattering become important. For both mechanisms the energy deposited in the sensitive region of the MOSFET is delivered mostly by secondary electrons. The photoelectric cross-section depends strongly on the target material, with the result of interface dose enhancement because the photoelectric cross section is different in the silicon (Si) and the silicumoxide $\left(\mathrm{SiO}_{2}\right)$ layer. More electrons will be produced in $\mathrm{Si}$, the higher cross section material, so more electrons will cross from $\mathrm{Si}$ into $\mathrm{SiO}_{2}$ than will cross the interface in the other direction. In the 
external beam radiotherapy range, where high energy photon beams (1 to $20 \mathrm{MV}$ ) or electron beams ( 6 to $20 \mathrm{MeV}$ ) are used, the observed energy dependence does not exceed $5 \%$ for both electron and photon irradiations ${ }^{9}, 10$.

Point detectors will always constitute an essential part of quality assurance in radiotherapy. Thermo-Luminescence Detectors (TLDs) and semiconductor detectors (silicon diodes) are the types of dosimeter most commonly employed for in vivo dosimetry purposes. Each of these detectors has their own advantages. MOSFETs and diodes provide dose information in real time. In case of discrepancy, this gives the ability to check each treatment parameter while the patient is still on the treatment couch. The advantage of both MOSFETs and TLDs is their small size, which allows for placement in a radiation beam without shadowing the dose in the patient or perturbing the beam characteristics and also allowing for placement in a catheter or a needle. The intrinsic precision of diodes is, however, superior to the MOSFET precision and tolerance levels for in vivo dosimetry using MOSFETs should be slightly widened with respect to measurements made with diodes ${ }^{3}$. For TLD dose measurements, an uncertainty of $2 \%(1 \mathrm{SD})$ has been reported ${ }^{11,12}$. These results are comparable to those of MOSFETs, but the use of TLD requires careful and time-consuming handling and annealing procedures to achieve these accurate results ${ }^{13,14}$. The superior precision of diodes, on the other hand, is compromised by the dependence of the diode response on temperature, dose-rate and accumulated dose. In addition, the dose-rate and temperature dependence change as the diode accumulates dose ${ }^{15}$. Due to the presence of a metallic build-up cap on the diode, the detector is energy dependent, and the sensitivity of the diode changes as a result of changes in source-surfacedistance (SSD), collimator size, and the presence of a wedge or tray ${ }^{16}$. To obtain accurate correction factors, an extensive series of phantom measurements is necessary. In contrast to diodes, MOSFETs have no dependence on dose-rate ${ }^{6}$ and do not show a sensitivity variation with accumulated dose ${ }^{3}$. Thereby, the MOSFET response of the single MOSFET dosimeter shows less dependence on temperature than the diode ${ }^{3}$. For the Linear 5ive MOSFET array, however, a temperature dependence of $11 \%$ was demonstrated by our group when increasing the temperature from room temperature to body temperature, which is higher than for diodes. The lifetime of the MOSFET detector is limited, compared to the life-time of diodes and TLDs. The extent of the MOSFET life-time depends on the sensitivity of the detector and the bias voltage used. Using standard MOSFET sensitivity and bias voltage, the detector's sensitivity is about $1 \mathrm{mV} / \mathrm{cG}$ in photon beams and the life-time is about $200 \mathrm{~Gy}$. The use of high sensitivity MOSFETs in combination with high sensitivity bias, results in a sensitivity of $9 \mathrm{mV} / \mathrm{cG}$ in photon beams, which reduces the life-time of the detector to about 70 Gy. These high sensitivity settings are however especially useful for measurements of small dose values (1-20 cGy) and for these dose values the number of measurements during the life time of the detector is quite reasonable. Thereby, the sensitivity differences for non-reference conditions between MOSFET detectors of a certain batch is within the intrinsic precision of the detector ${ }^{3}$. As a result the life-time of 
a new MOSFET is only reduced by the calibration of the detector; no additional phantom measurements for correction factor determination are required. A schematic overview comparing advantages and disadvantages of (micro) MOSFET, diode and TLD is given in table 1.The choice of a radiotherapy department for a point dosimeter suitable for in vivo dose measurements depends on the goal of the in vivo dose measurement protocol. When strict tolerance levels are important, diodes are suitable, however require a larger workload at the initial phase of its use. When tolerance levels are less strict, e.g, when there is a lack of staff, MOSFET detectors will be superior, because of the simplicity of the system and the less extensive procedure to implement the detector in clinical practice.

\begin{tabular}{|c|c|c|}
\hline Dosimeter & advantages & disadvantages \\
\hline \multirow[t]{7}{*}{ (micro) MOSFET } & small sensitive volume & limited life-time \\
\hline & small physical size & limited intrinsic presicion \\
\hline & real time dose information & \\
\hline & simple dose read-out & \\
\hline & neglectible beam pertubation & \\
\hline & lack of correction factors & \\
\hline & energy independent & \\
\hline \multirow[t]{6}{*}{ Diode } & high intrinsic presicion & energy dependent \\
\hline & high sensitivity & temperature dependence \\
\hline & real time dose information & dose-rate dependence \\
\hline & simple dose read-out & $\begin{array}{l}\text { dependence of response on } \\
\text { accumulated dose }\end{array}$ \\
\hline & & $\begin{array}{l}\text { sensitivity changes as a result of } \\
\text { changes in source-surface-distance } \\
\text { (SSD), collimator size, and the presence } \\
\text { of a wedge or tray }\end{array}$ \\
\hline & & $\begin{array}{l}\text { special care needed to ensure constancy } \\
\text { of response }\end{array}$ \\
\hline \multirow[t]{6}{*}{ TLD } & desired shape and size & no real time dose information \\
\hline & cheap & $\begin{array}{l}\text { time consuming procedure for dose read- } \\
\text { out }\end{array}$ \\
\hline & neglectible beam pertubation & limited intrinsic presicion \\
\hline & shape and size is variable & signal erased during readout \\
\hline & extended life-time & easy to lose reading \\
\hline & $\begin{array}{l}\text { many TLDs can be exposed in single } \\
\text { exposure }\end{array}$ & accurate results require care \\
\hline
\end{tabular}

Table 1: Schematic comparison of properties of (micro) MOSFET, diode and TLD. 
Besides the use of point dosimeters, the interest in using Electronic Portal Imaging Devices (EPIDs) for in vivo dose measurements is increasing over the last years. EPIDs measure transit dose values, which can be correlated to the dose at a point in a patient. With such a system 2D and even 3D dose information can be obtained ${ }^{17}$. Because of this large amount of dose information, EPIDs have advantages compared to point dosimeters for use as in vivo dosimetry dosimeter in photon beams. For electron beam radiotherapy, brachytherapy or special photon beam treatment techniques, such as extended field radiotherapy (e.g. TBI) the use of EPIDs is limited. In techniques applying adjacent fields, EPID dosimetry can give a lot of dose information; however special software is necessary for adding the dose values of the separate beams accurately. Furthermore, the electronic system along the edges of the EPID is sensitive to radiation. Exposure of this frame to the direct radiation beam should therefore be avoided. This means that for beams having large field sizes, beams at extended distance or very asymmetric beams, EPID dosimetry is not possible. To our experience, about $13 \%$ of the treatment fields cannot be imaged as a result of this aspect. MOSFET dosimeters have proven to be suitable detectors under these circumstances ${ }^{18-20}$. In the MAASTRO CLINIC, MOSFET entrance dosimetry is performed in the situations where EPI dosimetry is not possible. Finally, MOSFETs are used as a backup system, when an EPID does not function correctly. Our protocol prescribes that the initial in vivo dose measurement should be performed within 5 treatment fractions after the start of the treatment, and this procedure is repeated for sequential treatment plans (boost plans or changes in the initial plan). In this thesis, the use of MOSFET dosimeters for routine in vivo dosimetry during photon beam treatments was not described, because in our institution EPIDs are used for routine in vivo dosimetry in these beams.

\subsubsection{In vivo dosimetry as part of a routine QA programme}

An important aspect of a quality assurance programme is a check of the actual dose delivered to the patient. In vivo dose measurements permit data to be collected of the dose actually delivered at points of interest. This requires a convenient dosimeter and a methodology to allow accurate determination of the dose. Entrance dose measurements present important information about the beam output, the number of monitor units, the presence and direction of a wedge and limited information about the patient set-up, e.g. SSD. Exit dose measurements, additionally, give supplementary information about the size and density of the irradiated part of the patient's body. The importance of QA in radiotherapy is generally accepted, due to the complexity and many steps involved in the radiotherapeutic process. However, the workload involved in developing and assessing an in vivo dosimetry programme, is often a problem for a radiotherapy department. The literature available on in vivo dosimetry mostly concerns its use during routine treatments using photon beams ${ }^{21-28}$. Literature based on in vivo dosimetry in electron beams is scarce 29,30 . 
In our institution we developed an in vivo dosimetry protocol using MOSFETs for all curative and elective photon and electron beam treatments. Due to the intensive use of MOSFETs for in vivo dosimetry purposes in our institution, special attention is therefore paid to making it easy to use in daily practice with an acceptable accuracy. The radiation therapy technologists perform the measurements and a user interface was developed in which the radiation therapy technologists have to type in some specific treatment data. This program automatically determines the deviation of the measurement as a percentage of the prescribed dose. In chapter 3 we described our in vivo dosimetry protocol for use in electron beams. In this procedure, entrance dose measurements are obtained. The presence of a build-up cap during entrance dose measurements in electron beams is not desirable because it results in a dose reduction beneath the detector. The effect of the cylindrical aluminum build-up cap surrounding diode detectors is described by Yaparpalvi et al. ${ }^{30}$ and Frye and Rustgi 29 . The first group demonstrated that the aluminum build-up cap surrounding the diode sensitive volume of a commercial diode causes significant dose reduction, most noticeable with smaller fields and lower energy beams. The reduction in dose ranged from $16 \%$ (for a $6 \mathrm{MeV}$ beam and a $3 \mathrm{~cm}$ diameter circular field) to $4 \%$ (for a $12 \mathrm{MeV}$ beam and a $10 \mathrm{x}$ $10 \mathrm{~cm}$ field). These authors inferred that frequent use of diodes is undesirable when treating a small field by low energy electrons, because it might result in appreciable reduction of dose to the target volume. Frye and Rustgi reported similar results. In their protocol electron beam in vivo dosimetry using diodes, is only applied for initial verification of multiple fraction $(\mathrm{N}>3$ ) treatments on fields with $0-25 \%$ of open field area custom blocking for cones $10 \times 10 \mathrm{~cm}^{2}$ or greater. In this way they reduce the dose perturbation due to the diode to $5 \%$ or less of the total prescribed dose. Finally, readings outside the acceptable range or anomalous readings (patient motion, diode falls off, etc.) are flagged for repeat measurements on phantom material only; no in vivo repeats are permitted. In chapter 3 we described a method to reduce the shadowing effect of the electron beam by the build-up covering the detector. We converted the reading of a MOSFET detector placed on the patient's skin without additional build-up material to the dose at the depth of dose maximum using predefined factors and demonstrated that this technique is well suited for routine dose verification in electron beams.

\subsubsection{In vivo dosimetry as a tool to validate a treatment technique}

The validation of a new treatment technique should encompass both phantom measurements and patient measurements when appropriate. Regarding phantom measurements, the basic step would be to measure dose distributions using simple, multipurpose phantoms. The next step would be to check dose distributions in more clinical relevant phantoms, such as anthropomorphic phantoms. Such an approach, i.e. from simple phantom measurements to more complex phantom configurations, simulates the overall accuracy of the new treatment procedure. Nevertheless, these 
phantom measurements do not reflect by definition the total accuracy of patient treatment delivery, since the patient him/herself is excluded from the process. In order to assess the overall accuracy of a new treatment procedure at the patient level, patient dose verification is an essential and complementary part of the validation procedure of a new treatment technique. In practice, however, the use of in vivo dosimetry to validate a treatment technique in clinical practice is uncommon in most radiotherapy centres.

In chapter 2 the validation of a junction field technique in daily clinical practice is described after the initial validation of each separate treatment step and linear accelerator performance related factors. In theory, junction of fields using a half-beam method is ideal, because there is no divergence of the beam edge at the split line. In practice however, as demonstrated in this study, significant dose variation at the junction can occur due to small uncertainties in the positioning of the jaws at the position of the central beam axis. A $10 \%$ deviation of the dose value around the $50 \%$ point on a beam profile is related to a misalignment of $1 \mathrm{~mm}$ at a SSD of $100 \mathrm{~cm}$ for one block or $0.5 \mathrm{~mm}$ per jaw when the deviation for both jaws is in the opposite direction. Our linac QA programme is, with respect to the accuracy of the jaw position along the split line, performed using film dosimetry and more recently using EPID dosimetry. A deviation less than $10 \%$ at the junction line is accepted. This linear accelerator related tolerance combined with patient movements during the treatment, sometimes resulted in large dose discrepancies along the split line. The in vivo measurements also demonstrated that the under- and overdosage that arises in the split line area, average out during a series of fractionated treatments. Attention has to be paid when only a small number of treatment fractions are prescribed, because dose deviations along the split line may then not average out. To be aware of these uncertainties, in vivo dosimetry is indispensable.

In vivo dose information gives the opportunity to improve a specific treatment technique. Thanks to our in vivo dosimetry results and the detected dose deviations, we optimized our dose calculation procedure for TBI treatments from a point dose calculation without using a TPS, to a 3D dose calculation using our commercial TPS, as described in chapter 4. The use of a 3D planning system provides us with dose information over all regions of interest thus allowing a reduction of the dose in the extremities by using a simple MLC technique, or by using compensators specific for an individual patient. The use of a multileaf collimator is a good tool to improve dose homogeneity in the patient's body, as demonstrated in this thesis. It has, however, not yet been implemented in clinical practice due to the lack of a digital transfer system converting TPS parameters to treatment parameters of the accelerator. Such a digital transfer system is important because in the TPS, the SSD can virtually be extended to $400 \mathrm{~cm}$ while the patient is at the centre of the 2 opposed beams. In clinical practice, the extended source midline distance of $400 \mathrm{~cm}$ is achieved by positioning the gantry at a fixed location at $274^{\circ}$, and positioning the couch alongside the treatment room 
wall. Parallel opposed beams are achieved by switching the patient's position between the two fields. As a result, gantry angles and collimator angles are converted from TPS parameters to treatment parameters. In case of a segmented technique using the MLC as a compensator, converting the gantry and collimator angles of both segmented and open fields from treatment planning parameters to the treatment parameters, is error prone. Using a digital transfer system will prevent possible misinterpretation of these data. Patient treatments calculated using this 3D planning technique were evaluated in clinical practice using in vivo dosimetry.

\subsubsection{In vivo dosimetry to check the dose in organs at risk}

In addition to disease free survival, quality of life is an important outcome of a radiotherapy treatment in patients having cancer. Quality of life is related to the dose in normal tissues and particularly in OARs. The aim of the treatment planning process is to restrict the dose in these organs. In practice, however, there are several factors that effect the planned dose in the OAR. Deviations between plannend and actual dose values may originate from uncertainties in the delineation of the OAR on the CT images, in the dose calculation, in accelerator performance related factors, and in patient positioning. Verification of both the geometry and dose distribution is needed to prevent the patient from errors that may result in exceeding the tolerance dose of the OAR.

In chapters 5 and 6 we demonstrated the suitability of a technique to validate the dose in the urethra, the organ at risk during brachytherapy of permanent prostate implants, using ${ }^{125}$ s seeds. In practice, however, in vivo dosimetry in brachytherapy is not easy to perform, due to the high dose gradients involved and the necessity of performing measurements inside a patient. We described a method for dose verification in a catheter, correcting the in vivo dose values for catheter absorption and temperature dependence. In vivo dose measurements performed directly after the implantation give the ability to check the treatment procedure and trace systematic errors in the treatment chain including the dose calculation performed by the TPS. This information can be used to improve the treatment technique and treatment procedure. The in vivo results can not be used for adaptation of the treatment plan of a particular patient, because the measurements are performed after the implantation of the seeds. Constant monitoring the dose-rate during the implantation, however, gives the opportunity to adapt the treatment plan when the dose in the urethra will be too high and prevents the patient from having urinary side effects caused by too high absorbed doses in this organ at risk. By continuing the measurements for a short period directly after the implantation of the last seeds, the final dose rate after the implantation is also obtained and these in vivo dose values can next be used for evaluation of systematic results. This means that constant dose monitoring during and directly after the implantation gives the ability to evaluate and adapt the treatment plan for a particular patient, and additionally can be used to check and improve the treatment technique 
and procedure. In addition to the use of in vivo dosimetry after TIPPB, this approach can also be used for other brachytherapy techniques, such as HDR brachytherapy of prostate cancer or other cancer sites.

\subsection{FUTURE PERSPECTIVES}

Data obtained using in vivo dosimetry are evaluated by comparing measured dose values to data calculated by the TPS. For an accurate comparison, it is important that the location of the in vivo dose measurement point is in agreement with its location in the TPS. In practice however, this is not always easy to achieve. In case of conventional external beams, the detector will generally be placed on the central axis of the beam. In the TPS this point is also easy to find. In case of half beams or fields where one or more collimator shields the central beam axis, this is more difficult. Deviations in the location of the point where the dose is calculated and measured may result in false-positive errors. This will be the case when fields having large dose gradients are applied, for instance when wedged fields, oblique incident beams, irregular surfaces or brachytherapy treatments are used. To reduce these false-positive errors in TBI treatments, our protocol describes that the locations of these points for in vivo dose measurements are carefully chosen in the treatment preparation phase. During the virtual simulation CT-procedure, marks are placed in pre-defined areas on the patient's skin. These marks are visible both during treatment planning and during the actual treatment. In conventional external beam radiotherapy, however, field positions, sizes and gantry angles are determined during treatment planning and information about the measurement location is not provided during the CT-based virtual simulation procedure. In these situations additional information about the location where the in vivo dose measurement is performed is important. For external beams, this can be achieved by using additional images obtained during the actual treatment fraction, to visualize the detector in the beam. Loading these images into the TPS will allow determining the dose at the exact detector location. In this way a separation between errors due to detector positioning inaccuracies and actual dose errors can be separated and the number of false-positive errors can be reduced. An example of this method is using a cone-beam CT scan made just before the actual treatment fraction with the detector positioned at the measuring position. Such a conebeam CT scan can be used for patient setup verification, but also provides, when loaded into the TPS, useful information about the dosimeter position in the actual treatment plan and the calculated dose at this position. To visualize a MOSFET detector on the images, an additional marker will be necessary for electron beams. In photon beams our MOSFET detectors are visible, due to the build-up cap on the detector. Besides the use of cone-beam CT for patient and dosimeter position verification, it can be used for dose calculation. The cone-beam information, loaded into the treatment planning system, gives the ability to calculate the actual absorbed 
dose in a treatment fraction with the patient in treatment position. Repeating this procedure for each treatment fraction gives information about the actual dose accumulated over the entire treatment provided the TPS has the ability to add dose distributions of single treatment fractions. However, one should be aware of the extra dose given with cone beam CT. A careful balance should be made between the additional dose given by one or a series of cone-beam CT scans and the additional information, according to the ALARA principle.

Additional imaging to visualize the detector will also be important for in vivo dose measurements during brachytherapy treatments, where the detector is placed in a catheter or needle in a patient. Due to the large dose gradients involved, knowledge of the exact position of the detector in the catheter or needle is important to allow a meaningful comparison to the dose calculation performed by the TPS. MOSFET detectors are however not visible on CT- and ultrasound images. In the studies described in this thesis we therefore used a dummy array, having markers close to each MOSFET, to visualize the MOSFET position during imaging. For the in vivo dose measurements these markers would affect the dose distribution. Therefore during the in vivo measurements the linear array was used having the disadvantage of introducing differences in the actual position of the linear array compared to the position of the dummy array in the catheter. A position tracking device might be useful for linking dosimeter position and dose values. This year (2009), Best Medical (Ottawa, Canada) will introduce the RADPOS system. This system will make it possible to link the position of the MOSFET detector in or on the patient to measured in vivo dose values. The dosimetry system is connected with a position-tracking device, having the possibility of accurately and real-time deriving the sensor position and orientation. The advantage of this system compared to imaging is the lack of administering additional dose to the patient's body. Especially when repeated measurements are performed this will be an important issue. Movements of the patient during the treatment, which affect the dose measurement, will be visible using this system. This is not the case when using imaging to locate the detector position before the actual treatment, because changes in position that may occur after the imaging process will not be visualized. On the other hand, a patient tracking system should be compatible with the TPS in order to link dose values and dosimeter position in the treatment room to coordinates in the TPS. A high geometric accuracy of such a system is therefore a prerequisite for its clinical use.

In this study in vivo dosimetry was used to check the entrance dose in vivo in seperate radiation beams. It would, however, also be of interest to check the dose in the target area itself. If entrance dose measurements alone are applied, this means that the entrance dose has to be converted to the corresponding target dose, using patient and treatment set-up information. A combination of entrance and exit dose measurements is a more accurate way of obtaining the target dose. Various methods are available to obtain the midline dose from entrance and exit dose values. These methods give 
generally good results when the patient is considered to be homogeneous, but in the presence of tissue inhomogeneities considerable deviations may occur. Positional shifts of these inhomogeneous tissue areas, located between the entrance point of the beam and the target area, will influence the dose in the target. Entrance in vivo dosimetry alone is not able to detect these errors. To determine the dose in the target area, exit dose information is needed in addition to the entrance dose data. This exit dose information can be obtained using a second point dosimeter; however, also a combination of entrance point dosimetry and transit EPID dosimetry is a method potentially suitable for this purpose. For an accurate dose determination in the target area, tissue density data of the patient during the actual treatment fraction is essential. This data can be obtained by using the EPID information or by performing an additional cone-beam CT scan just before the fraction in which the dose measurement is performed.

In this study in vivo dose values were compared to calculated dose values thus yielding an opportunity to prevent errors in the treatment process. In vivo dosimetry, however, is also useful to link dose values to clinical outcome. Collecting systematic dose data provides us with a large amount of dose information for the different treatment sites. The follow-up procedure of the patients after the actual treatment allows us to establish clinical outcome, side effects and quality of life, which gives us the ability to link absorbed dose data to clinical outcome for each treatment site and treatment technique. This information is essential to improve current radiotherapy treatments and for future developments in radiotherapy. 


\section{REFERENCES}

1. Cygler JE, Saoudi A, Perry G, et al. Feasibility study of using MOSFET detectors for in vivo dosimetry during permanent low-dose-rate prostate implants. Radiother Oncol 2006;80:296-301.

2. Roshau JN, Hintenlang DE. Characterization of the angular response of an "isotropic" MOSFET dosimeter. Health Phys 2003;84:376-379.

3. Jornet $\mathrm{N}$, Carrasco $\mathrm{P}$, Jurado D, et al. Comparison study of MOSFET detectors and diodes for entrance in vivo dosimetry in 18 MV x-ray beams. Med Phys 2004;31:25342542.

4. Ramani R, Russell S, O'Brien P. Clinical dosimetry using MOSFETs. Int J Radiat Oncol Biol Phys 1997;37:959-964.

5. Ferreira IH, Dutreix A, Bridier $A$, et al. The ESTRO-QUALity assurance network (EQUAL). Radiother Oncol 2000;55:273-284.

6. Soubra M, Cygler J, Mackay G. Evaluation of a dual bias dual metal oxide-silicon semiconductor field effect transistor detector as radiation dosimeter. Med Phys 1994;21:567-572.

7. Ramaseshan R, Kohli KS, Zhang TJ, et al. Performance characteristics of a microMOSFET as an in vivo dosimeter in radiation therapy. Phys Med Biol 2004;49:4031-4048.

8. Lavallee MC, Gingras L, Beaulieu L. Energy and integrated dose dependence of MOSFET dosimeter sensitivity for irradiation energies between $30 \mathrm{kV}$ and 60Co. Med Phys 2006;33:3683-3689.

9. Oldham T.R., J.M. M. Comparison of $60 \mathrm{Co}$ Response and $10 \mathrm{keV}$ X-Ray Response in MOS capacitors. EEE Trans. Nucl. Sci NSI 1983;30.

10. Tallon R.W., Ackerman M.R, Kemp W.T., et al. A Comparison Of loinizing Radiation Damage in MOSFET's from 60Co Gamma Rays, 0.5 To 22 Mev Protons and 1 to 7 MeV Electronics. IEEE Trans. Nucl. Sci. 1985;32:4393.

11. McGhee P, Humphreys $S$, Dunscombe $P$. An efficient approach to routine $T L$ dosimetry. Med Dosim 1993;18:187-191.

12. Fairbanks EJ, DeWerd LA. Thermoluminescent characteristics of LiF:Mg,Ti from three manufacturers. Med Phys 1993;20:729-731.

13. Wood JJ, Mayles WP. Factors affecting the precision of TLD dose measurements using an automatic TLD reader. Phys Med Biol 1995;40:309-313.

14. Kron $\mathrm{T}$, Butson $\mathrm{M}$, Wong $\mathrm{T}$, et al. Readout of thermoluminescence dosimetry chips using a contact planchet heater. Australas Phys Eng Sci Med 1993;16:137-142.

15. Jornet N, Ribas M, Eudaldo T. In vivo dosimetry: intercomparison between p-type based and n-type based diodes for the 16-25 MV energy range. Med Phys 2000;27:1287-1293.

16. Ding W, Patterson W, Tremethick $L$, et al. Calibration of entrance dose measurement for an in vivo dosimetry programme. Australas Radiol 1995;39:369-374.

17. van Elmpt W, McDermott L, Nijsten S, et al. A literature review of electronic portal imaging for radiotherapy dosimetry. Radiother Oncol 2008;88:289-309.

18. Bloemen-van Gurp EJ, Mijnheer BJ, Verschueren TA, et al. Total body irradiation, toward optimal individual delivery: dose evaluation with metal oxide field effect 
transistors, thermoluminescence detectors, and a treatment planning system. Int J Radiat Oncol Biol Phys 2007;69:1297-1304.

19. Bloemen-van Gurp EJ, Minken AW, Mijnheer BJ, et al. Clinical implementation of MOSFET detectors for dosimetry in electron beams. Radiother Oncol 2006;80:288295.

20. Bloemen-van Gurp E, du Bois W, Visser P, et al. Clinical dosimetry with MOSFET dosimeters to determine the dose along the field junction in a split beam technique. Radiother Oncol 2003;67:351-357.

21. Cozzi L, Fogliata-Cozzi A. Quality assurance in radiation oncology. A study of feasibility and impact on action levels of an in vivo dosimetry program during breast cancer irradiation. Radiother Oncol 1998;47:29-36.

22. Essers M, Mijnheer BJ. In vivo dosimetry during external photon beam radiotherapy. Int J Radiat Oncol Biol Phys 1999;43:245-259.

23. Fiorino $C$, Corletto $D$, Mangili $P$, et al. Quality assurance by systematic in vivo dosimetry: results on a large cohort of patients. Radiother Oncol 2000;56:85-95.

24. Fontenla DP, Yaparpalvi R, Chui CS, et al. The use of diode dosimetry in quality improvement of patient care in radiation therapy. Med Dosim 1996;21:235-241.

25. Higgins PD, Alaei P, Gerbi BJ, et al. In vivo diode dosimetry for routine quality assurance in IMRT. Med Phys 2003;30:3118-3123.

26. Kalef-Ezra JA, Boziari A, Litsas J, et al. Thermoluminescence dosimetry for quality assurance in radiation therapy. Radiat Prot Dosimetry 2002;101:403-405.

27. Millwater CJ, MacLeod AS, Thwaites DI. In vivo semiconductor dosimetry as part of routine quality assurance. Br J Radiol 1998;71:661-668.

28. Voordeckers M, Goossens H, Rutten J, et al. The implementation of in vivo dosimetry in a small radiotherapy department. Radiother Oncol 1998;47:45-48.

29. Frye DM, Rustgi SN. Diode verification of routine electron-beam treatments. Med Dosim 1999;24:43-48.

30. Yaparpalvi R, Fontenla DP, Vikram B. Clinical experience with routine diode dosimetry for electron beam radiotherapy. Int J Radiat Oncol Biol Phys 2000;48:12591265. 

Summary 



\section{IN VIVO DOSIMETRY IN RADIOTHERAPY}

In this thesis, an overview is provided of MOSFET applications in various fields of radiotherapy. The pilot studies described in this thesis resulted in the implementation of in vivo dosimetry in routine clinical practice. The various steps in this process starting with the calibration of the detector, the phantom measurements to test the procedure and finally the patient measurements were described for a wide range of applications in both internal and external beam radiotherapy.

\section{In vivo dosimetry as part of a routine quality assurance PROGRAMME}

In chapter 3 we describe the use of MOSFET dosimeters for entrance in vivo dose verification in electron beams. The goal of this study was to design a method for routine entrance in vivo dose verification, without build-up material on the detector, to prevent the target volume from shadowing effects of the build-up material. In this procedure, we convert surface readings of the detector to absolute dose values at depth of dose maximum, using conversion factors obtained by a comprehensive set of phantom measurements. In this procedure the MOSFET reading, converted to dose at depth of dose maximum, was compared to the dose prescribed at this depth. An additional correction for the distance between the surface and the applicator, a correction for fields having sides smaller than $6 \mathrm{~cm}$ and for irregular field shape is recommended in this procedure for accurate dose determination. The procedure was tested in clinical practice for 40 patients, mostly treated for breast tumours. The results demonstrated deviations from the prescribed dose with a mean difference of $-0.7 \%$ and a standard deviation of $2.9 \%$. From this study it can be concluded that performing dose measurements with MOSFET detectors placed on the patient's skin without additional build-up is a well suited technique for routine dose verification in electron beams if appropriate conversion and correction factors are applied.

\section{In vivo dosimetry as a tool to validate a new treatment technique}

In chapters 2 and 4 results are presented of studies of in vivo dose measurements to validate a new treatment technique in clinical practice.

The first goal of the study described in chapter 2 was to determine the accuracy of MOSFET measurements for dose determinations in a junction field technique using half beams. The second goal was to evaluate the dose distribution across the split line using MOSFET dosimeters. The results obtained from a comprehensive set of phantom measurements using MOSFET detectors, were compared to the results of ionisation chamber and photographic film measurements. Next, the influence of factors related to the mechanical properties and QA procedures of the linear accelerator were discussed. These results were used to evaluate patient measurements, where the 
accuracy of the junction was determined on 5 patients receiving loco-regional treatment of breast cancer during 10 treatment fractions. From this study we concluded:

(1) significant dose deviations in the junction area average out over repeated treatments;

(2) a single measurement in the junction area should not lead to any action;

(3) the linac QA procedure to check the dose at the junction line should simulate the clinical situation as much as possible;

(4) MOSFET dosimeters overestimate dose values in the penumbra region.

In the study described in chapter 4, in vivo dosimetry was used to verify a new 3dimensional (3D)-dose calculation procedure of our Total Body Irradiation (TBI) technique. To obtain relevant dose calculation information, a total body CT scan was performed and loaded into our commercial Treatment Planning System (TPS). A 3Ddose distribution was generated, after the initial validation of this TPS for extended distance radiotherapy. To verify the new procedure in the clinic, in vivo dosimetry was performed for 5 patients, at five locations on the patient's body. Entrance and exit dose values were converted to midline doses, using conversion factors previously determined with phantom measurements. These measurements demonstrated that MOSFET and TLD dose values agreed within 3.0\%, and MOSFET and TPS data even within $0.5 \%$. The convolution algorithm of the TPS, which is routinely applied in the clinic, overestimated the dose in the lung region. Using a superposition algorithm reduced the calculated lung dose with approximately $3 \%$. The dose inhomogeneity predicted by the TPS can be reduced by using a simple IMRT technique. From this chapter, we conclude that:

(1) using a TPS for dose calculation of TBI gives good insight in over- and underdosage in the patient and the influence of patient positioning on dose homogeneity;

(2) MOSFETs are suitable dosimeters for in vivo dosimetry purposes during TBI if appropriate conversion factors are applied.

\section{In vivo dosimetry to check the dose in organs at risk}

In chapters 5 and 6 the application of MOSFET dosimeters for dose verification in organs at risk during brachytherapy is described.

In chapter 5 results are presented of a study of a new linear 5-MOSFET-array dosimeter for in vivo dose verification of permanent prostate implants using ${ }^{125}$. Phantom measurements were carried out to validate the measurement technique, to determine the measurement accuracy and to define action levels for clinical measurements. Patient measurements were performed directly after the implantation procedure. A MOSFET-array was placed in the urinary catheter, in order to provide useful information about the actual dose in the urethra, to evaluate the implantation 
technique, to evaluate the accuracy of the TPS and to link dose distributions in OARs to clinical side effects. A CT-scan was made and dose values, calculated by the treatment planning system, were compared to in vivo dose values measured with MOSFET-dosimeters. The in vivo results were corrected for temperature dependence of the MOSFET-array response and photon attenuation in the catheter. In vivo dose values were obtained for 17 patients. In the high dose region (>100 Gy) calculated and measured dose values agreed within $1.7 \% \pm 10.7 \%$ (1SD). In the low dose region outside the prostate (<100 Gy) larger deviations occurred. From this study, we concluded that MOSFET-detectors are suitable for in vivo dosimetry during brachytherapy of prostate cancer using ${ }^{125}$. An action level of $\pm 16 \%$ (2 SD) for detection of errors in the implantation procedure is achievable after validation of the detector system and measurement conditions.

The goal of the study described in chapter 6 was to investigate the feasibility of a linear-MOSFET-array for real-time dosimetry during permanent prostate brachytherapy, to check the complete treatment chain and to prevent patients from getting urinary complications as a consequence of too high doses in this organ at risk. The results of phantom measurements, described in chapter 5 , were used for this study. Additional phantom measurements were performed to determine the optimal conditions for clinical measurements. An overall validation of the measurement method was performed with a simulation of clinical practice in a phantom. Real-time measurements were performed for 5 patients during the implant. To evaluate if the real-time urethra dose with the ultrasound transducer in the rectum and the patient in treatment position is a reference for the total accumulated dose, additional measurements were performed in the recovery. Real-time measurements and measurements performed in the recovery agreed very well, illustrating that the ultrasound transducer in the rectum and patient positioning do not influence the dose in the urethra. Real-time in vivo dose values obtained during the implantation are therefore representative for the total accumulated dose in the urethra at these positions. For the 5 patients the dose-rates during and after the implantation were below the maximum tolerable dose-rate of the urethra and adaptation of the planned seed distribution was therefore not necessary. The conclusion of the study presented in this chapter is that real-time dosimetry using a MOSFET-array is a promising technique to evaluate the dose in the urethra during the implantation of using ${ }^{125}$ seeds for prostate irradiation.

Finally, chapter 7 gives general conclusions that can be derived from this thesis and a broad discussion of the results from our studies with regard to the use of in vivo dosimetry in radiotherapy. Furthermore future perspectives are provided. 

Samenvatting 



\section{IN VIVO DOSIMETRIE BIJ RADIOTHERAPIE}

In dit proefschrift wordt een overzicht gegeven van de mogelijkheden van in vivo dosisverificatie met MOSFET dosimeters op het brede toepassingsgebied van de radiotherapie. Verschillende pilot-studies, beschreven in dit proefschrift, hebben geresulteerd in routinematige uitvoering van in vivo dosimetrie in de klinische praktijk. De verschillende stappen in het acceptatie- en implementatieproces van een nieuwe dosimeter, met als doel geprotocolleerde in vivo dosimetrie in de dagelijkse praktijk, worden in dit proefschrift beschreven voor een breed scala aan toepassingen voor zowel inwendige als uitwendige radiotherapie. Kort samengevat bestaat dit proces uit het kalibreren van de detector, het verrichten van fantoommetingen voor het testen van het meetinstrument, het vaststellen van de procedure voor het verrichten van patiëntmetingen en het testen van deze procedure bij een geselecteerde patiëntengroep.

\section{In vivo dosimetrie als onderdeel van een kwaliteitsborgingprogramma}

In hoofdstuk 3 beschrijven we het gebruik van MOSFET stralingsmeters voor intrede in vivo dosisverificatie bij elektronenbundels. Het doel van deze studie was het ontwerpen van een methode voor routinematige in vivo dosisverificatie bij elektronenbundels, zonder gebruik te maken van opbouwmateriaal op de detector. Dit om dosisreductie achter het opbouwmateriaal te voorkomen. In de procedure, beschreven in dit hoofdstuk, werden dosismetingen aan het oppervlak met behulp van omrekeningsfactoren omgezet naar absolute dosiswaarden op de diepte van het dosismaximum. Deze omrekeningsfactoren werden verkregen door een uitgebreide reeks van fantoommetingen. De MOSFET-waarneming, omgerekend naar dosis op diepte van het dosismaximum, werd vervolgens vergeleken met de voorgeschreven dosis op deze diepte. Een correctie voor de afstand tussen het oppervlak en de applicator, een correctie voor kleine velden met zijden kleiner dan $6 \mathrm{~cm}$ en een correctie voor onregelmatige veldvorm, worden aanbevolen in deze procedure voor een nauwkeurige dosisbepaling. De procedure werd getest in de klinische praktijk voor 40 patiënten, voornamelijk patiënten die werden behandeld voor borstkanker. De resultaten toonden een gemiddeld verschil van $-0,7 \%$ en een standaarddeviatie van $2,9 \%$ ten opzichte van de voorgeschreven dosis. Uit deze studie kan worden geconcludeerd dat het uitvoeren van dosismetingen met MOSFET-detectoren, geplaatst op de huid van de patiënt zonder extra opbouwmateriaal op de detector, een geschikte techniek is voor routine dosisverificatie bij elektronenbundels, mits nauwkeurige conversie en correctiefactoren worden toegepast. 


\section{In vivo dosimetrie als instrument voor het valideren van een nieuwe behandelingstechniek}

In de hoofdstukken 2 en 4 worden de resultaten van studies gepresenteerd waarbij in vivo dosimetrie wordt toegepast voor het valideren van een nieuwe behandelingstechniek.

Het primaire doel van de studie beschreven in hoofdstuk 2 was het vaststellen van de nauwkeurigheid van MOSFET-metingen voor dosisverificatie in de regio waar twee halve bundels op elkaar aangesloten worden. Het uiteindelijke doel was om de dosisverdeling over het aansluitgebied te verifiëren met behulp MOSFETstralingsmeters. De MOSFET-resultaten bij een uitgebreide reeks van fantoommetingen, werden vergeleken met de resultaten van ionisatiekamerdetectoren en fotografische filmmetingen. In dit hoofdstuk wordt tevens de invloed van factoren in verband met de mechanische eigenschappen en QA-procedures van de lineaire versneller besproken. Deze informatie werd gebruikt voor het evalueren van de meetresultaten bij een groep van 5 patiënten. Deze patiënten ondergingen een locoregionale radiotherapie behandeling, waarbij met behulp van een halve bundeltechniek zowel het borstweefsel als de axillaire/supraclaviculaire klieren bestraald werden. Gedurende 10 behandelfracties werden MOSFET metingen verricht om de nauwkeurigheid van de dosis in het aansluitgebied van de bundels te bepalen. De conclusies van deze studie luiden:

(1) significante dosisafwijkingen in het aansluitgebied middelen zich uit over herhaalde behandelingsfracties

(2) een enkele meting in het aansluitgebied mag nooit leiden tot een actie

(3) de linac QA procedure om de nauwkeurigheid van de aansluiting te bepalen moet de klinische situatie zoveel mogelijk benaderen

(4) MOSFET stralingsmeters overschatten de dosiswaarden in het halfschaduw gebied.

In hoofdstuk 4 wordt in vivo dosimetrie toegepast om een nieuwe driedimensionale (3D)-dosisberekeningstechniek voor een totale lichaamsbestralingtechniek (TBI), te verifiëren en te controleren. Voor het verkrijgen van relevante dosisberekeningsinformatie, werd bij de patiënten in deze studie een totale lichaam CT-scan gemaakt. De beeldinformatie werd geladen in een commerciëel Treatment Planning System (TPS), nadat dit systeem eerst gevalideerd was voor lange afstand radiotherapie. Een 3D-dosisverdeling werd daarbij gegenereerd. In vivo dosimetrie werd verricht bij 5 patiënten, op vijf locaties op het lichaam van de patiënt met zowel MOSFET als TL-detectoren. Intrede- en uittrede dosiswaarden werden omgerekend naar dosiswaarden midden in de patiënt, waarbij gebruik werd gemaakt van omrekeningsfactoren. Deze omrekeningsfactoren werden vastgesteld door middel van een uitgebreide set fantoommetingen. MOSFET en TLD dosiswaarden komen binnen $3,0 \%$ overeen. MOSFET en TPS dosiswaarden komen binnen $0,5 \%$ overeen. Het convolutie-algoritme van het TPS, dat routinematig wordt toegepast in de kliniek, 
overschat de dosis in de longregio. Met behulp van een superpositie algoritme wordt de longdosis nauwkeuriger benaderd. De dosisinhomogeniteiten in het lichaam van de patiënt, zoals berekent met behulp van het TPS, kunnen worden verminderd door gebruik te maken van een eenvoudige IMRT-techniek. Uit dit hoofdstuk concluderen we dat:

(1) het gebruik van een TPS voor dosisberekening bij TBI een goed inzicht geeft in de over- en onderdoseringen in de patiënt en inzicht geeft in de invloed van patiëntpositionering op dosishomogeniteit

(2) MOSFET stralingsmeters zijn geschikt voor in vivo dosimetrie doeleinden tijdens TBI.

\section{In vivo dosimetrie als instrument om de dosis in risico organen te bepalen}

In de hoofdstukken 5 en 6, wordt de toepassing van MOSFET stralingsmeters voor dosisverificatie in risico organen beschreven.

In hoofdstuk 5 worden de resultaten van een studie gepresenteerd waarbij een nieuwe lineaire 5-MOSFET array-dosimeter toegepast wordt voor in vivo dosisverificatie bij permanente prostaatimplantaten met jodium-125 zaadjes. Fantoommetingen werden verricht voor het valideren van de meettechniek, voor het bepalen van de meetnauwkeurigheid en voor het testen van de detector voor de klinische metingen. Patiëntmetingen werden verricht bij 17 patiënten in de recovery, direct na de implantatieprocedure. Een MOSFET-array werd in de urinekatheter geplaatst, met als doel het verifiëren van de actuele dosis in de urineleider. Deze informatie werd gebruikt voor het evalueren van de implantatietechniek en het beoordelen van het TPS. Informatie over de ureterdosis geeft bovendien de mogelijkheid om actuele dosisinformatie te koppelen aan klinische bijwerkingen. $\mathrm{Na}$ de in vivo metingen werd een CT-scan gemaakt. Dosiswaarden berekend door het TPS op basis van de post-implant CT-scan, werden vergeleken met de in vivo dosiswaarden gemeten met de MOSFET-stralingsmeters, na correctie van de in vivo resultaten voor de temperatuursafhankelijkheid van de MOSFET-array respons en voor absorptie van de katheterwand. In het hoge dosisgebied (> $100 \mathrm{~Gy}$ ) komen berekende en gemeten dosis binnen 1,7\% $\pm 10,7 \%$ (1SD) overeen. In het lage dosisgebied ( $<100$ Gy), de regio buiten de prostaat, werden grotere afwijkingen gemeten. Uit deze studie hebben we geconcludeerd dat MOSFET-detectoren geschikt zijn voor in vivo dosimetrie na brachytherapie met jodium-125 bij prostaatkanker. Een actielevel van \pm $16 \%$ (2 SD) voor het opsporen van fouten in de implantatieprocedure is haalbaar na validatie van de detector en meetomstandigheden. In het hoge dosisgebied zijn kleinere actielevels haalbaar.

In hoofdstuk 6 word het gebruik van een lineair-MOSFET-array getest voor real-time dosimetrie tijdens brachytherapie van de prostaat. Het primaire doel van de real-time metingen gedurende de implantatie van de jodium- 125 zaadjes, is het verifiëren van de 
dosis in de urineleider. Dit geeft de mogelijkheid de verdeling van de zaadjes aan te passen op basis van de in vivo meetresultaten met als doel te hoge stralingsdoses en urinaire complicaties te voorkomen. Het secundaire doel is het controleren van de behandelketen en de implantatietechniek. De resultaten van fantoommetingen, beschreven in hoofdstuk 5, werden gebruikt voor deze studie. Aanvullende fantoommetingen werden verricht om de optimale omstandigheden voor de klinische metingen vast te stellen. Tevens werd een simulatiemeting verricht om de klinische praktijk na te bootsen. Real-time metingen werden verricht bij 5 patiënten in de operatiekamer tijdens de implantatie van de zaadjes. Om te beoordelen of de real-time dosis in de urineleider, met de echografie-transducer in de endeldarm en de patiënt in behandelpositie, een referentie is voor de totale cumulatieve dosis in de urineleider, werden aanvullende metingen verricht in de recovery. Real-time metingen en metingen verricht in de recovery kwamen goed overeen, hetgeen illustreert dat de echografietransducer in het rectum en positionering van de patiënt geen aantoonbare invloed hebben op de dosis in de urineleider. Real-time in vivo dosiswaarden verkregen tijdens de implantatie, zijn dus representatief voor de totale cumulatieve dosis in de urineleider op deze posities. Voor alle 5 patiënten bleken de dosis-waarden tijdens en na de implantatie lager dan de maximaal toelaatbare dosiswaarde van de urineleider. Aanpassing van de geplande zaadverdeling in de prostaat was derhalve niet nodig. De conclusie van deze studie is dat real-time dosimetrie met behulp van een MOSFETarray een veelbelovende techniek is voor de evaluatie van de dosis in de urineleider tijdens de implantatie van jodium-125 zaadjes bij prostaat bestraling.

Tenslotte geeft hoofdstuk 7 de algemene conclusies weer die kunnen worden afgeleid uit dit proefschrift. Er wordt een uitgebreid overzicht gegeven van de resultaten van de studies beschreven in dit proefschrift en het gebruik van in vivo dosimetrie in de radiotherapie. Bovendien worden mogelijke toekomstperspectieven beschreven, waardoor in vivo dosisverificatie in de toekomst verbeterd kan worden. 
Dankwoord 



\section{DANKWOORD}

Het gevoel dat ik nu heb, bij het afronden van mijn proefschrift en het schrijven van dit dankwoord, is te vergelijken met gevoel van een bergbeklimmer die zojuist de top heeft bereikt. Bij het bereiken van deze top heb ik niet de meest voor de hand liggende route genomen. Nee, het was een route met verschillende omzwervingen via landweggetjes (opleidingen) en langs dorpjes (diverse functies). Echter, deze omzwervingen vormden geen verspilde tijd en moeite. Integendeel, ze resulteerden in een brede ervaring en een ruim blikveld. Deze ervaring heb ik goed kunnen gebruiken bij het beklimmen van de berg en het tot stand brengen van mijn uiteindelijke doel, het bereiken van de top ofwel het afronden van mijn proefschrift. Bovendien heeft het de reis een stuk aangenamer gemaakt. Op de top van de berg, sta ik nu na te genieten van alle ervaringen die ik de afgelopen jaren heb mogen opdoen, het geweldige uitzicht, maar vooral het triomfantelijke gevoel dat ik er, al is het dan via een alternatieve weg, gekomen ben. Nu ik echter boven aan de berg sta zie ik dat ik eigenlijk weer aan de voet van een volgende berg sta, een veel hogere berg. Het gevoel van triomf verdwijnt langzaam naar de achtergrond. Al snel maakt het gevoel van triomf echter plaats voor wilskracht. Wilskracht om mijn bergschoenen weer op te poetsen en verder te gaan, een nieuwe weg in te slaan in een poging ook deze tweede berg te bedwingen. Te kijken of ik ooit ook deze top zal bereiken of dat ik wellicht onderweg een aangename plek tegenkom waar ik een tijd blijf hangen....

Bij het beklimmen van deze berg heb ik vele mensen ontmoet die ik wil bedanken voor de mogelijkheden die ze me gegeven hebben de top te bereiken. Omdat het onmogelijk is om iedereen persoonlijk te vernoemen, wil ik starten om alle mensen te bedanken die direct maar ook indirect met mijn promotie te maken hebben gehad en die ik hieronder niet noem.

Promotor, prof. Philippe Lambin en copromotoren dr. Ben Mijnheer en dr. ir. André Dekker wil ik bedanken voor hun bijdrage en ondersteuning bij mijn promotie. Beste Philippe, jij bent naast opdrachtgever tevens inspirator geweest voor het verrichten van in vivo dosimetrie in MAASTRO CLINIC. Dankzij jou kreeg ik de mogelijk me te gaan richten op onderzoek en iets te gaan doen met de opleiding waar ik op dat moment mee bezig was. Je enthousiasme was aanstekelijk en maakte dat ik net een stapje harder liep. Jij was ook degene die me stimuleerde uiteindelijk te gaan promoveren. Ik kan me nog precies herinneren wat je hierover zei: " Het stelt niet zo veel voor, je publiceert 3 artikelen en schrijft een inleiding en samenvatting en klaar ben je....". De berg bleek uiteindelijk iets hoger dan je in eerste instantie suggereerde, maar spijt van mijn keuze heb ik nooit gehad. Bedankt hiervoor.

Beste Ben Mijnheer, waar jij ook was (en dat was regelmatig in het buitenland), je was altijd bereikbaar voor vragen en het lezen van een manuscript. Van de verschillende 
coauteurs, betrokken bij de diverse publicaties, was jij steeds degene die het eerste reageerde op een manuscriptopzet. Je was altijd op een positieve manier kritisch. Maandelijks kwam je naar Maastricht voor overleg en evaluatie van mijn onderzoeksresultaten. Omdat ik je voor je bezoek de meest recente data wilde sturen, kreeg jij dus vaak pas de avond voordat je naar Maastricht af zou reizen een e-mail met de daarbij horende documenten. Je hebt je dus nooit hoeven vervelen in de trein op weg naar Maastricht ....

André Dekker, gedurende mijn promotie ben jij als copromotor bij mijn onderzoek betrokken en heb je een deel van de begeleiding op je genomen. Tijdens de wekelijkse vergadering van de "Dose Guided Radiotherapy-groep" (DGRT-groep) waarin de onderzoeksresultaten van de verschillende onderzoekers besproken werden was je altijd goed in het stellen van kritische vragen en het meedenken naar oplossingen en resultaten. Bedankt voor deze kritische blik. De overige leden van de DGRT-groep, Wouter, Bas en Steven, ook jullie wil ik bedanken voor het meedenken in de verschillende fasen van het onderzoek en het brainstormen over de behaalde resultaten.

Huub Backes, als hoofd para- en peri-medische groep en later innovatiemanager was jij er mede verantwoordelijk voor dat ik de mogelijkheid kreeg een Master opleiding te volgen naast mijn gewone werkzaamheden als radiotherapeutisch laborant. Deze studiejaren waren niet eenvoudig doordat ik tegelijkertijd naast een fulltime baan, 20 uur in de week zelfstudie moest verrichten en ook nog eens samen met mijn partner het oude huis dat we gekocht hadden volledig moesten verbouwen. Maar Huub, jij hebt altijd het volste vertrouwen in me gehad dat ik deze studie binnen de geplande tijd en met voldoende resultaat zou afronden. Mijn dank hiervoor!

Bij het afstuderen aan de Master opleiding bleek een van mijn begeleiders een bekende te zijn, Ria Korthoeve, Radiotherapeut Oncoloog en docent MBRT. Ria, jij noemde mij in je praatje tijdens de diploma uitreiking "een voorddaverende trein die direct door gaat naar het eindstation, zonder te stoppen voor de tussenliggende stations" en jij was degene die tijdens het begeleiden van mijn afstuderen begon over promoveren. Je vond dat wel iets voor mij. Dit was echter iets waar ik op dat moment zelf nog niet over nagedacht had. Maar wat wel in mijn hoofd bleef nasluimeren. De eerste belangstelling was gewekt. Ik slaagde binnen de benodigde tijd met een "Cum Laude" eindresultaat. Beste Ria, bedankt voor de prettige begeleiding tijdens mijn afstuderen, maar vooral voor het vertrouwen dat je me gaf ten aanzien van de toekomst.

Wim DuBois was als Radiotherapeut Oncoloog bij MAASTRO CLINIC betrokken bij de opstartfase van het dosimetry project. Wim, samen met jou ben ik in andere instituten gaan kijken als oriëntatie op het project. Je had in je werkzaamheden zo je eigen werkwijze, maar je was wel degene die vaak bij me binnenliep om te vragen hoe de stand van zaken was en om me te ondersteunen. Tevens heb jij een belangrijke 
bijdrage geleverd aan mijn eerste publicatie. Bedankt voor dit alles. Helaas, na relatief korte tijd verliet je MAASTRO CLINIC om elders aan de slag te gaan. Ik moest vanaf die tijd zonder jou verder.

Ook laïn Bruinvis wil ik in deze niet vergeten. laïn, jij was destijds hoofd van de klinisch fysica groep, en hebt er aan bijgedragen dat ik van de "paramedische groep" (laborantengroep) naar de klinisch fysica groep ben "verhuist", waardoor ik meer mogelijkheden had mezelf te ontwikkelen en mijn onderzoek verder uit te breiden. Tevens heb je een belangrijke bijdrage gehad aan mijn eerste publicatie. Echter, ook jij verliet na relatief korte tijd MAASTRO CLINIC. Je opvolger was André Minken, een klinisch fysicus die al in MAASTRO CLINIC werkte. André, jij en ik werkten al voor je aanstelling als hoofd Klinisch Fysica Groep regelmatig samen. Ik wil je bedanken voor je adviezen met betrekking tot het verrichten van de vele metingen die er gedaan moesten worden en je bijdrage aan één van de artikelen.

Peter Visser, klinisch fysicus in MAASTRO CLINIC, bedankt voor je hulp bij het opzetten van "MosDos", een database voor het verwerken en registreren van de in vivo meetresultaten. Lars Murrer, eveneens klinisch fysicus in MAASTRO CLINIC, jou wil ik bedanken voor je adviezen betreffende de metingen bij brachytherapy en je bijdrage aan mijn laatste publicaties. En natuurlijk ook voor het feit dat je mijn paranimf wil zijn! De klinisch fysisch medewerkers van MAASTRO CLINIC wil ik bedanken voor hun bijdrage bij het verrichten van de vele fantoommetingen voor het valideren van de MOSFET dosimeter. In het bijzonder wil ik Sem Krasuski bedanken. Sem, bedankt voor de fijne samenwerking rondom het onderzoek bij TBI en de vele kalibraties die je verricht hebt. Uiteraard wil ik ook de rest van de KFG-groep bedanken voor de fijne samenwerking en de gezellige uitstapjes.

Tevens wil ik een aantal radiotherapeut-oncologen van MAASTRO CLINIC bedanken voor hun bijdrage aan dit proefschrift. Tom Verschueren, bedankt voor de samenwerking tijdens het TBI project. Je had het volste vertrouwen in de dosimetrie resultaten, die uiteindelijk hebben geleid tot de nieuwe berekeningsmethode. Francis van Gils en Ludy Lutgens, bedankt voor jullie medewerking tijdens de dosismetingen op de uitslaapafdeling en in de operatiekamer tijdens het inbrengen van de radioactieve zaadjes. Francis, jou in het bijzonder wil ik nog bedanken voor je bijdrage aan de laatste 2 artikelen. En niet te vergeten Jos Jager. Jos, als ik je tegen kwam in de gang maakte je altijd de grap dat je wel mijn paranimf wilde worden.... Nu is het dan bijna zover, ik kon het niet laten je echt te vragen.

Ook de laboranten werkzaam bij MAASTRO CLINIC: bedankt voor het verrichten van de vele in vivo patiëntmetingen op de verschillende lineaire versnellers.

Verder wil ik de studenten van de opleiding Biometrie die hebben meegeholpen aan mijn onderzoek bedanken voor hun bijdrage bij het verrichten van de metingen voor het onderzoek MOSFET dosimetrie bij brachy. Gerben Kaas, Raoul Daemen, Joost van Elk en Björk Haanstra, bedankt voor jullie goede inzet. 
Cary Dehing-Oberye, statistisch analist in MAASTRO CLINIC, bedankt voor je adviezen in de beginfase van mijn onderzoek op het gebied van de statistische analyse van de resultaten.

Het afronden van een proefschrift kost enorm veel tijd. Secretariële ondersteuning is hierbij onmisbaar. Rian, Simone en Sonia, mijn dank voor jullie bijdrage.

De leden van de beoordelingscommissie, prof. Wildberger, prof. Heijmen, prof. Verhaegen en dr. Venselaar wil ik bedanken voor alle tijd die ze hebben gespendeerd aan het bestuderen en beoordelen van dit proefschrift.

Abdelbasset Hallil, klinisch fysicus en "Director of Research \& Development" bij Best Medical, (Ottawa, Canada) het bedrijf waarvan Thomson and Nielsen, de fabrikant en ontwikkelaar van de in deze thesis toegepaste MOSFET systemen, een onderdeel is. Beste "Basset", bedankt voor het verlenen van de dosimetertechnische informatie rondom de MOSFET dosimeters. Door deze informatie kon ik mijn onderzoeksresultaten voldoende onderbouwen.

Tenslotte wil ik mijn ouders bedanken voor de goede steun gedurende mijn studie- en promotieperiode. Pap, mam, zonder jullie was ik nooit zo ver gekomen. Tevens wil ik mijn ouders en schoonouders bedanken voor de goede en liefdevolle opvang van mijn kinderen op dagen dat ik naar een cursus of congres moest of gewoon tijdens werkdagen. Met een geruster hart kon ik de kinderen niet achterlaten.

Als laatste, lieve Eric, bedankt voor je steun en begrip tijdens mijn studie en promotieperiode. Je hebt nooit geklaagd als je er alleen voor stond met de kinderen of als ik naar een cursus of congres was. Lucas en Elise, jullie aanwezigheid maakte dat ik in drukke tijden het werk kon relativeren. Bedankt voor jullie steun.

En tot slot, op de ruggen van Lady en Joy kon ik heerlijk ontspannen en al het werk van me laten afglijden... 


\section{Curriculum Vitae}





\section{CURRICULUM VITAE}

Esther Bloemen-van Gurp werd geboren op 30 maart 1970 te Deventer. Na anderhalf jaar verhuisde het gezin naar het Brabantse Oisterwijk, waar Esther het grootste deel van haar jeugd doorbracht. In 1989 voltooide zij haar HAVO diploma aan het Durendael College te Oisterwijk. Aansluitend startte zij het Hogere Laboratorium Onderwijs (HLO) aan de Hogeschool te Eindhoven. Na 1 jaar wisselde zij, op eigen initiatief, het Hogere Laboratorium Onderwijs in voor de nieuwe HBO opleiding "Medisch Beeldvormende en Radiotherapeutische Technieken" (MBRT), tevens aan de Hogeschool te Eindhoven. In 1994 behaalde ze haar Bachelorgraad. In oktober 1994 kon ze beginnen bij het voormalig Radiotherapeutisch Instituut Limburg te Heerlen (tegenwoordig MAASTRO CLINIC te Maastricht), als radiotherapeutisch laborante. Het Brabantse landschap werd ingeruild voor de Limburgse heuvels. In 1999 startte ze naast haar werkzaamheden als radiotherapeutisch laborante, met de 2-jarige opleiding "Master of Arts in Health" aan de Hogeschool te Eindhoven. In 2001 werd de Mastergraad behaald met een cum laude resultaat. Mede dankzij deze studie kreeg Esther de mogelijkheid om zich binnen MAASTRO CLINIC verder te ontwikkelen op het gebied van onderzoek, met als aandachtsgebied in vivo dosimetrie met MOSFET dosimeters. Ze volgde de cursus "Physics for clinical radiotherapy" te Leuven, de statistiekcursussen "Statistics part 1: introduction in clinical statistical methods" en "Statistics part 2: regression and variance analysis", te Maastricht en de NVVO oncologie cursus "Introduction to the clinical and fundamental Oncology". Daarnaast behartigde Esther de belangen van de medewerkers van MAASTRO CLINIC, als voorzitter van de ondernemingsraad. In $2006 \mathrm{kwam}$ aan deze nevenfunctie een einde, toen de onderzoeksfunctie omgezet werd naar een promotieplaats, met Prof. Philippe Lambin als promotor en Dr. Ben Mijnheer en Dr. Andre Dekker als co-promotor. In 2008 werd haar inzet op het gebied van in vivo dosimetrie bij brachytherapy beloond met het behalen van een internationale award, de Jack Fowler award, uitgereikt tijdens de $27^{\text {th }}$ Annual ESTRO meeting in Göteborg (Zweden). Op 1 april 2009, na het afronden van haar promotieonderzoek, is Esther gestart als post-doctoraal onderzoeker bij MAASTRO CLINIC met als onderwerp 3D ultrasound voor dosisgestuurde radiotherapie. Esther Bloemen-van Gurp is getrouwd met Eric Bloemen en heeft twee kinderen, Lucas en Elise. 

Publications 



\section{PUBLICATIONS}

1. Bloemen-van Gurp E.J., du Bois W.F.J., Visser P.A., Bruinvis I., Jalink D., Hermans J. and Lambin P. Clinical dosimetry with MOSFET dosimeters to determine the dose along the field junction in a split beam technique. Radiotherapy and Oncology 67 (2003) 351-357.

2. Bloemen-van Gurp E.J., Minken A.W.H., Mijnheer B.J., Dehing- Oberye C.J.G. and Lambin P. Clinical implementation of MOSFET detectors for dosimetry in electron beams. Radiotherapy and Oncology 80 (2006) 288-295.

3. Bloemen-van Gurp E.J., Mijnheer B.J., Verschueren T.A.M. and Lambin P. Total body irradiation, towards optimal individual delivery: dose evaluation with MOSFETs, TLDs and a treatment planning system. Int. J. Radiation Oncology Biol. Phys., Vol. 69, No. 4, pp. 1297-1304, 2007.

4. Bloemen-van Gurp E.J., Murrer L.H.P., Haanstra B.K.C., van Gils F.C.J.M., Dekker A.L.A.J., Mijnheer B.J. and Lambin P. In vivo dosimetry using a linear MOSFET-array dosimeter to determine the urethra dose in ${ }^{125}$ permanent prostate implants. Int. J. Radiation Oncology Biol. Phys., Vol. 73, No. 1, pp. 314-321, 2009.

5. Bloemen-van Gurp E.J., Haanstra B.K.C., Murrer L.H.P, van Gils F.C.J.M., Dekker A.L..A.J., Mijnheer B.J. and Lambin P. Real-time dosimetry with a linear MOSFET array to evaluate the urethra dose during permanent implant brachytherapy using lodine-125. Int. J. Radiation Oncology Biol. Phys (in press).

\section{PUBLISHED ABSTRACTS}

1. Bloemen-van Gurp E.J., Minken A.W.H., Visser P.A., du Bois W.F.J., Lambin P. Implementation of MOSFET dosimetry in daily practice. $21^{\text {st }}$ Annual ESTRO Meeting, Praha, Czech Republic, 2002.

2. Du Bois W.F.J., Bloemen-van Gurp E.J., Visser P.A., Bruinvis I., Jalink D., Hermans J., Lambin P., Minken A.W.H. Commissioning of a MOSFET-TNRD-50 dosimetry system for routine measurements of $6 \mathrm{MV}$ and $10 \mathrm{MV}$ external beam photon radiation treatments. $21^{\text {st }}$ Annual ESTRO Meeting, Praha, Czech Republic, 2002.

3. Bloemen-van Gurp E.J., Wulms C., Lambin P., Mijnheer B.J, Minken A.W.H. Correction factors for accurate use of MOSFETs for entrance in-vivo dosimetry in photon beams. 23 ${ }^{\text {rd }}$ ESTRO Meeting, Amsterdam, The Netherlands, 2004. 
4. Bloemen van Gurp E.J., Minken A.W.H., Dehing-Oberije C.J.G, Lambin P., Mijnheer B.J. The implementation of MOSFET detectors for clinical dosimetry in electron beams. 23 ${ }^{\text {rd }}$ ESTRO Meeting, Amsterdam, The Netherlands, 2004.

5. Bloemen- van Gurp E.J., Mijnheer B.J., Minken A.W.H., Verschueren T.A.M, Murrer L.H.P., Lambin P. Total body irradiation, towards optimal individual delivery: dose evaluation with MOSFETs, TLDs and A Treatment planning system. $25^{\text {th }}$ ESTRO Meeting, Leipzig, Germany, 2006.

6. Bloemen-van Gurp E.J., Murrer L.H.P., van Elk J., Mijnheer B.J., van Gils F.C.J.M, Lambin P. Accuracy of in vivo dose verfification in brachy therapy using microMOSFET dosimeters. 26 th ESTRO Meeting, Barcelona, Spain, 2007.

7. Haanstra B.K.C., Bloemen- van Gurp E.J., Murrer L.H.P., van Gils F.C.J.M., Pijls R.J.M.W., Dekker A.L.A.J., Mijnheer B.J. and Lambin P. In-vivo dosimetry at interstitial prostate irradiation. 23rd Annual Symposium of the Belgian Hospital Physicists Association, Leuven, Belgium, 2008.

8. Bloemen- van Gurp E.J., Haanstra B.K.C., Murrer L.H.P., van Gils F.C.J.M., Pijls R.J.M.W., Dekker A.L.A.J., Mijnheer B.J. and Lambin P. In-vivo dosimetry in the urethra after interstitial prostate irradiation. 27th ESTRO Meeting, Göteborg, Sweden, 2008.

9. Bloemen-van Gurp E.J., Lutgens L., Murrer L.H.P., Verhaegen F.J.W., Dekker A.L.A.J., Mijnheer B.J. and Lambin P. In vivo dosimetry in HDR brachytherapy for cervix cancer. $10^{\text {th }}$ Bienial ESTRO meeting on physics and radiation technology for clinical radiotherapy, Maastricht, The Netherlands. 
Achievements and implementations 



\section{ACHIEVEMENTS AND IMPLEMENTATIONS}

The research work performed by Esther Bloemen-van Gurp resulted in a broad application of MOSFET in vivo dosimetry in MAASTRO CLINIC.

In vivo dosimetry in MAASTRO CLINIC is applied to all patients treated with a curative intent, using photon or electron beams. In standard photon beam treatments, portal dose images are the primary tool for dose verification. For special treatment techniques, such as $\mathrm{TBI}$, large fields, very asymmetric fields and larger distance treatments, portal dosimetry is not possible, due to the radio-sensitive electronics in the edge of the EPID device. In these situations, MOSFETs are used for dose verification. Furthermore, MOSFET dosimeters are used for dose verification in electron beams, because of the lack of signal for EPID dosimetry.

MOSFET in vivo dose measurements are performed for approximately 1500 treatment fields each year. Our clinical database contains the measurement data of more than 9000 photon and electron beams.

\section{In vivo dosimetry in photon beams}

In 2002, routine in vivo dosimetry was implemented in daily practice in MAASTRO CLINIC for photon beam radiotherapy treatments having curative treatment intent. For each photon beam an in vivo measurement is performed by the radiation technologists, as a quality check of the treatment procedure. The measurements, performed under full build-up to achieve equilibrium conditions, are compared to the calculated dose data, calculated using the TPS. To convert the in vivo dose data and compare the corrected dose values to the calculated dose values, Esther and her group developed a home-made software program "MOSDOS", to process the data and to calculate the deviation between calculated and in vivo dose data. In addition, she developed a decision tool for the radiation technologists and the physicians to standardize the actions when the deviation between the in vivo dose value and the calculated dose value exceeds the tolerance level (figure 1). Furthermore, action levels were defined, based on their clinical experience. These action levels ranged from $7 \%$ in standard beam configurations up to $10 \%$ for breast treatments and very asymmetric radiation fields. 


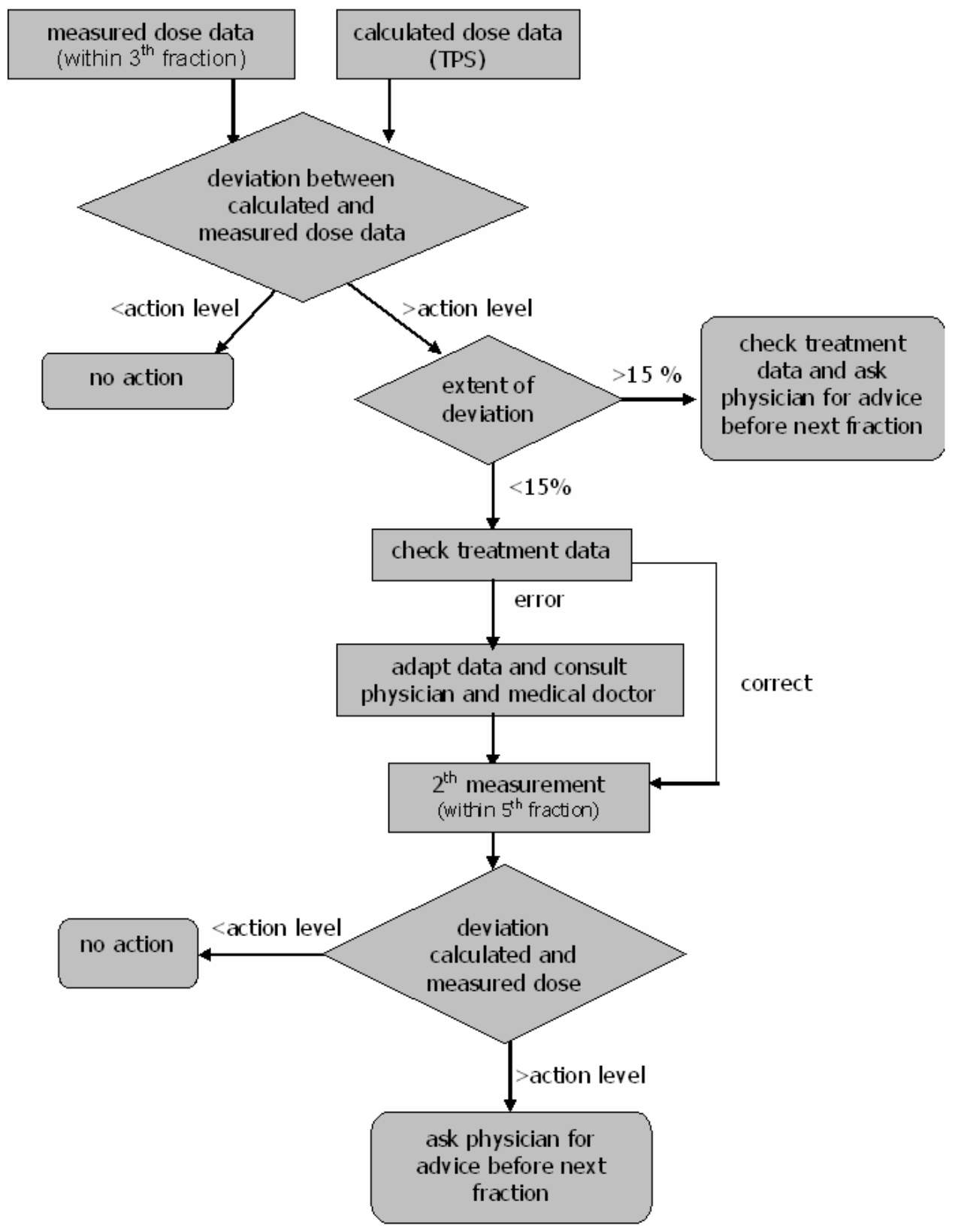

Figure 1: Decision tool for the radiation technologists and the physicians to standardize the workflow of the in vivo dose measurements. 


\section{In vivo dosimetry in electron beams}

After the implementation of photon beam in vivo dosimetry in the clinic, Esther continued her work for electron beam configurations. This work focused on the response of MOSFET detectors in electron beams and the feasibility of measuring without build-up on the detector. The optimal conditions for the clinical measurements and conversion factors to convert in vivo surface dose to dose at depth of $D_{\max }$, were established. The clinical introduction of routine electron beam in vivo dosimetry started in 2003. MOSDOS was further developed to convert the in vivo surface dose to dose values at depth of dose maximum, and to calculate dose deviations between in vivo and calculated dose data.

\section{In vivo dosimetry in total body irradiations}

In 2003, in addition to the work in electron beams, the use of MOSFET dosimeters under TBI circumstances was investigated. This work resulted in 2004 in the routine application of MOSFETS for in vivo dosimetry in TBI treatments. Entrance and exit dose measurements were converted to midline dose values using predefined factors, and the in vivo results were compared with the prescribed dose. As a result of the in vivo dose data and the simple dose calculation protocol used routinely in our clinic, Esther investigated the probability to use the clinical TPS for 3D-dose calculation at extended distance, in order to obtain more accurate dose calculations. In 2006 this study resulted in a new, fully digitalized, protocol for TBI in MAASTRO CLINIC (figure 2) using a 3D-dose calculation method and the opportunity of using the MLC as a compensator.

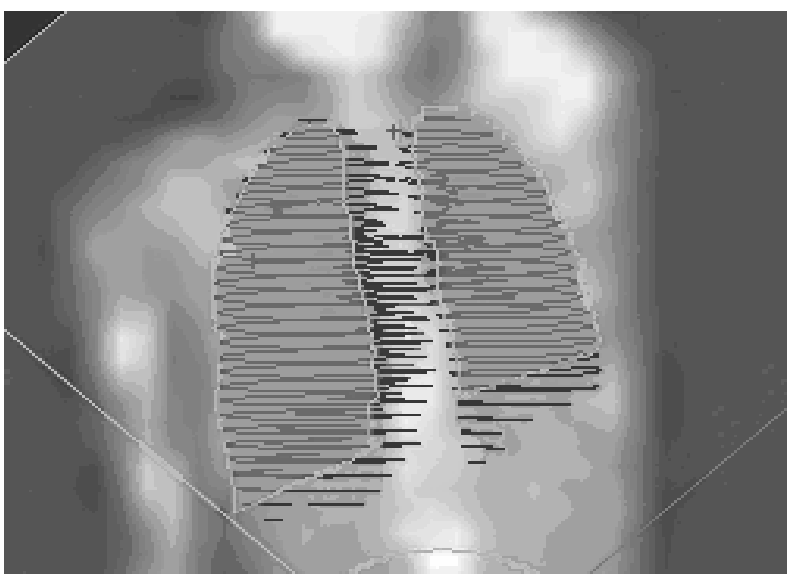

Figure 2: Design of the lung blocks for the treatment of TBI patients. 


\section{In vivo dosimetry in brachytherapy}

In 2006 Esther extended her work in the area of brachytherapy. The sensitivity of MOSFET detectors for ${ }^{125}$ was investigated for both reference and non-reference conditions. The results were used for in vivo dose measurements in the patient, during and after permanent implant brachytherapy using ${ }^{125}$. This study demonstrated that in vivo dosimetry using MOSFET dosimeters is a very useful tool for image guided brachytherapy.

In 2008, this study was awarded with the Jack Fowler Award, an award that recognizes an outstanding junior investigator for exceptional work in radiation oncology, medical physics and/ or radiobiology, and was presented during the $27^{\text {th }}$ Annual ESTRO meeting in Göteborg, Sweden.

\section{University Award}

Miss Esther Bloemen - Van Gurp

MAASTRO Clinic, Maastricht, The Netherlands

\#*ESTRO-Fowler Award ***

In-vivo Dosimetry in the Urethra after Interstitial Prostate Irradiation.

My PhD work is based on in-vivo dosimetry using MOSFET detectors, in both external beam radiotherapy and brachytherapy and I will finish my thesis next year.

The work leading to this award is based on in vivo dosimetry in brachytherapy. This is quite a challenge due to the high dose gradients and the low photon energies involved. Our group presented the results of a phantom and patient study, to validate the microMOSFET for dose verification after permanent 1251 implants and to evaluate the detector

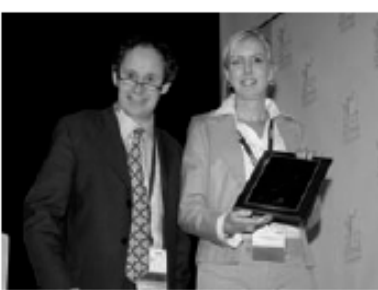

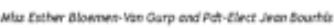
under clinical use in order to determine the dose in the urethra after the implant.

Phantom measurements were performed to investigate the sensitivity of the micro-MOSFET under varying conditions. A reference measurement was performed to simulate the clinical situation using a clinical plan and a custom-made phantom. Measured and calculated dose values were compared. Patient measurements were performed in 17 patients, directly after the implant procedure using a high sensitivity linear 5ive array (TN-502LA) positioned in the urinary catheter. The in-vivo dose values were extrapolated to the total dose at these positions, using the known half-life time of the 1251, and the results were compared with the calculated dose values at these positions using the TPS.

The results of this study demonstrated that a temperature correction of 119 , the deviation between calibration temperature and patient temperature, is necessary to correct the in vivo results. A correction for the attenuation caused by the catheter is also used. Patient in-vivo measurements demonstrated a mean deviation of $-5.6 \% \pm 17.6 \%$. A deviation of $-1.19 \% \pm 9.9 \%$ (1SD) was observed in the higher dose region (>100 Gy). The larger deviations in the regions distant from the seeds (low dose) are mostly due to the larger distance between the detector and the seeds resulting in the increasing influence of inhomogeneities, which is not calculated accurately by the TPS. The global deviation, expressing the dose relative to the highest dose, is however a better way to express the dose in the low dose region (figure 2), resulting in a deviation of $-4.8 \% \pm 115$ (15D).

The conclusion is that MOSFETS are potentially suitable for in-vivo dosimetry after interstitial prostate implantation and can contribute considerably to the overall quality assurance of permanent 1251 prostate implants. The high dose region is accurate enough to safeguard the dose to the urethra $(200 \mathrm{gy})$. For adequate interpretation some factors still have to be investigated such as the influence of inhomogeneity correction and inter-seed attenuation in the low dose region. 
170 
\begin{tabular}{l|l} 
Notes & 171
\end{tabular} 
172 
174 
176 
